\title{
Structure Determination of a Chloroenyne from Laurencia majuscula Using Computational Methods and Total Synthesis
}

\author{
Erin D. Shepherd, ${ }^{\dagger}$ Bryony S. Dyson, ${ }^{\dagger}$ William E. Hak, ${ }^{\dagger}$ Quynh Nhu N. Nguyen, ${ }^{\dagger}$ Miseon Lee, ${ }^{\ddagger}$ \\ Mi Jung Kim, ${ }^{\ddagger}$ Te-ik Sohn, ${ }^{\ddagger}$ Deukjoon Kim, ${ }^{*},{ }^{\ddagger}$ Jonathan W. Burton, ${ }^{* \dagger}$ and Robert S. Paton ${ }^{*}, \S_{\odot}$ \\ ${ }^{\dagger}$ Chemistry Research Laboratory, Department of Chemistry, University of Oxford, Mansfield Road, Oxford OX1 3TA, United \\ Kingdom \\ ${ }^{\ddagger}$ The Research Institute of Pharmaceutical Sciences, College of Pharmacy, Seoul National University, Seoul 151-742, Korea \\ ${ }^{\S}$ Department of Chemistry, Colorado State University, Fort Collins, Colorado 80523, United States
}

\section{Supporting Information}
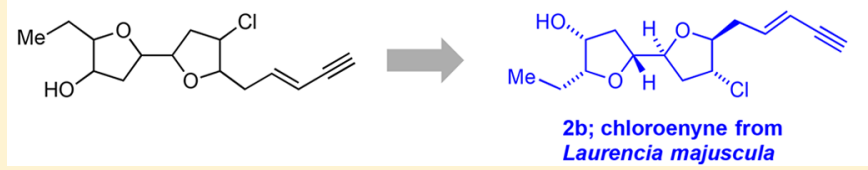

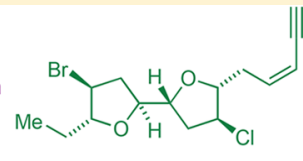

(Z)-3a; (Z)-notoryne

ABSTRACT: Despite numerous advances in spectroscopic methods through the latter part of the 20th century, the unequivocal structure determination of natural products can remain challenging, and inevitably, incorrect structures appear in the literature. Computational methods that allow the accurate prediction of NMR chemical shifts have emerged as a powerful addition to the toolbox of methods available for the structure determination of small organic molecules. Herein, we report the structure determination of a small, stereochemically rich natural product from Laurencia majuscula using the powerful combination of computational methods and total synthesis, along with the structure confirmation of notoryne, using the same approach. Additionally, we synthesized three further diastereomers of the L. majuscula enyne and have demonstrated that computations are able to distinguish each of the four synthetic diastereomers from the 32 possible diastereomers of the natural product. Key to the success of this work is to analyze the computational data to provide the greatest distinction between each diastereomer, by identifying chemical shifts that are most sensitive to changes in relative stereochemistry. The success of the computational methods in the structure determination of stereochemically rich, flexible organic molecules will allow all involved in structure determination to use these methods with confidence.

\section{INTRODUCTION}

Elucidating the structures of natural products that are available only in very small quantities is often exceptionally difficult, highlighted by the high number of structure revisions reported every year in the chemical literature. ${ }^{1}$ Unequivocal establishment of molecular structure may be possible using singlecrystal X-ray diffraction; however, for molecules that will not crystallize or that form poorly diffracting crystals, alternative methods must be used. ${ }^{2}$ Nuclear magnetic resonance (NMR) spectroscopy remains one of the primary means of molecular structure determination. Comparison of computationally predicted NMR chemical shifts, coupling constants, and internuclear distances have also emerged as a powerful and reliable way to assess the likelihood of a putative structure being correct. NMR computations have thus been used to establish relative stereochemistry, ${ }^{3}$ to confirm or reassign proposed natural product structures, ${ }^{4}$ to characterize the identity of a side product, ${ }^{5}$ and in conformational assignment of cyclic peptides. ${ }^{6}$ However, this approach can become particularly challenging for molecules containing multiple stereogenic centers and with a high degree of conformational flexibility. In such cases, weighted ensemble averages must be considered in comparison against experimentally observed NMR parameters. In the case of complex structures such as baulamycin, hundreds and thousands of conformations may be relevant at room temperature. ${ }^{7}$ Although elegant synthetic approaches have been developed to access multiple diastereomers of a target molecule, ${ }^{8}$ the ability to predict the most plausible relative and absolute configuration of flexible natural product structures is desirable. Flexible, halogenated natural products epitomize this challenge and have been variously misassigned. ${ }^{9}$

Comparison of experimental NMR parameters, such as chemical shifts, against calculated values can reveal obvious discrepancies and raise doubts about a proposed structure. ${ }^{4 a}$ Furthermore, several measures have been used to quantify the "best match" from several putative structures with respect to an experimental spectrum. In particular, statistical parameters developed in the Goodman group, namely, $\mathrm{CP} 3{ }^{10}$ and DP4, ${ }^{11}$ use prior knowledge of the underlying empirical error distribution of computational predictions to assign statistical

Received: January 18, 2019

Published: April 12, 2019 
confidence values to a particular structural assignment. This has led to their widespread adoption in computational natural product assignments. ${ }^{12}$ These metrics emphasize the importance of ensuring a narrow underlying prediction error as this allows greater significance to be attached to the differences between incorrect structures and experiment. More confident assignments can be made as a result. Empirical linear scaling, aided greatly through contributions from the Tantillo group and the CHESHIRE repository, ${ }^{13}$ has contributed to this as have modified DP4 models and the use of different internal standards against which chemical shifts are referenced. ${ }^{14}$ However, molecules with many accessible conformers can give rise to larger prediction errors, ${ }^{3}$ making the use of these now standard tools more precarious. Herein, we report the full structure determination of a $2,2^{\prime}$-bifuranyl chloroenyne natural product isolated from Laurencia majuscula (2) using the powerful combination of biosynthetic postulates, density functional theory (DFT) calculations of NMR chemical shifts, and total synthesis. In addition, we report the synthesis of all four biosynthetically relevant diastereomers of $2(\mathbf{2 a}-\mathbf{d})$ and show that comparison of the experimental ${ }^{1} \mathrm{H}$ and ${ }^{13} \mathrm{C}$ NMR data for any of these four diastereomers with the computed ${ }^{1} \mathrm{H}$ and ${ }^{13} \mathrm{C}$ NMR data for all 32 diastereomers of the natural product allows each specific biomimetic diastereomer to be identified. Finally, we report the structure confirmation of $(Z)$ notoryne as ( $Z$ )-3a using the above combined approach of quantum chemical calculations coupled with two distinct total syntheses.

Recently, we assigned the full structures of two $2,2^{\prime}$-bifuranyl natural products using the powerful combination of biosynthetic postulates, DFT calculations of NMR chemical shifts, and total synthesis. We used this combined approach to fully elucidate the structure of elatenyne ${ }^{15}$ and to reassign the stereostructure of laurefurenynes $\mathrm{A}$ and $\mathrm{B}^{16}$ Previously, we demonstrated that the gross structure of a chloroenyne isolated from Laurencia majuscula, originally assigned as a pyrano[3,2b]pyran $(\mathbf{1})^{17}$ on the basis of extensive NMR experiments and comparison with the NMR data of the dactomelynes ${ }^{18}$ and of the originally assigned pyrano[3,2-b]pyran structure of elatenyne, ${ }^{19}$ was actually a $2,2^{\prime}$-bifuranyl 2 (Chart 1 ). ${ }^{15 a, b}$ The 2,2'-bifuranyl 2 contains six stereocenters, with the full structure of the natural product being one of 32 possible diastereomers. We aimed to solve the complete stereostructure of this natural product using the combined approach which we had previously found successful, namely, biosynthetic postulates coupled with DFT calculations of NMR chemical shifts and total synthesis.

Clues from Biosynthesis. Algae of the genus Laurencia produce a vast array of structurally diverse $C_{15}$ halogenated natural products. Elatenyne, ${ }^{19,20}$ notoryne (3), ${ }^{21}$ laurendecumenyne $\mathrm{B},{ }^{20,22}$ laurefurenynes $\mathrm{A}$ and $\mathrm{B},{ }^{23}$ and the above chloroenyne from L. majuscula $2^{17}$ are currently the only $2,2^{\prime}$ bifuranyl natural products that have been isolated from Laurencia spp. Notoryne 3 was the first of these 2,2'-bifuranyls to be isolated, and its structure was determined through extensive chemical degradation and by comparison with chemical degradation products of laurencin. ${ }^{21 a}$ A plausible biosynthesis of notoryne $\mathbf{3 a}$ was proposed by Suzuki and by Fukuzawa and Murai. ${ }^{21 a, 24}$ For (3Z)-notoryne ( $\left.Z\right)$-3a, $(3 Z, 12 E, R, R)$-laurediol $4 \mathrm{a}^{25}$ is converted into (3Z)-deacetyllaurencin $6 \mathbf{a}$ via bromonium ion formation and cyclization (Figure 1a). ${ }^{26}$
Chart 1. Proposed Structures of Chloroenyne from $L$. majuscula (Relative Configurations) along with the Structures of $(Z)$ - and (E)-Notoryne (Absolute Configurations)
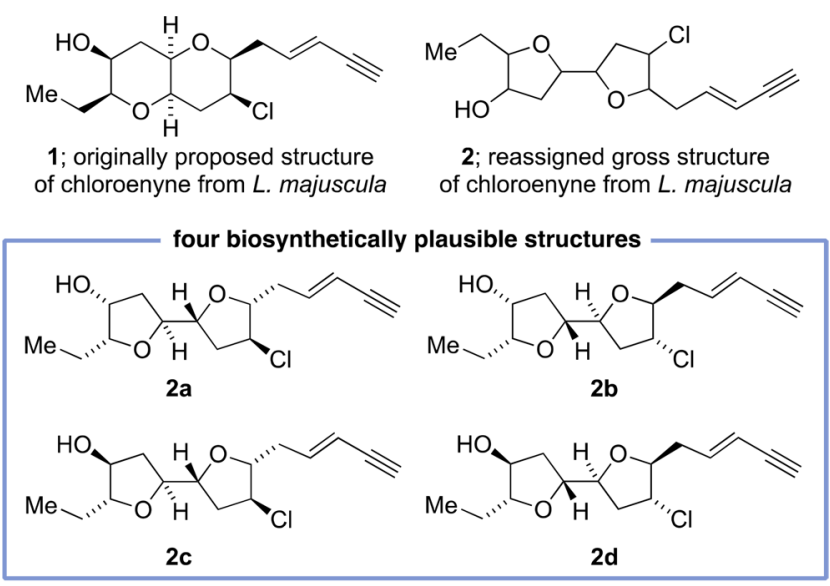

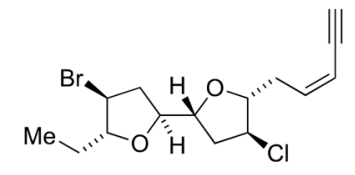

(Z)-3a; (Z)-notoryne

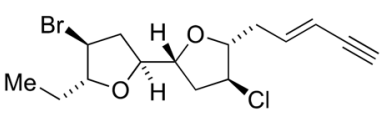

(E)-3a; (E)-notoryne
Further bromoetherification of (3Z)-deacetyllaurencin 6a gives the (3Z)-dibromide 8a, prelaurefucin, via 7a. Transannular displacement of bromide gives (3Z)-tricyclic oxonium ion 9a, which on opening at C-7 with chloride gives (3Z)notoryne (3Z)-3a. As we previously proposed for the biosynthesis of laurefurenynes $\mathrm{A}$ and $\mathrm{B},{ }^{16 \mathrm{a}}$ displacement of bromide by water (or a water equivalent) ${ }^{27}$ would give rise to $2 \mathrm{a}$ as a potential stereostructure for the chloroenyne from $L$. majuscula. Based on this proposed biosynthesis of notoryne 3a, we recently proposed a biosynthesis of elatenyne, laurendecumenyne $\mathrm{B},{ }^{15 \mathrm{~d}}$ and laurefurenynes $\mathrm{A}$ and $\mathrm{B},{ }^{16 \mathrm{a}}$ which begins from a different diastereomer of the laurediols $(4 \mathbf{b})^{25}$ and proceeds via the natural product bromofucin $\mathbf{8 b} .^{28}$ Here, laurediol $\mathbf{4 b}$ undergoes bromoetherification to give a diastereomer of deacetyllaurencin $\mathbf{6 b}$, which undergoes further bromoetherification to give bromofucin $\mathbf{8 b}$ (Figure $1 \mathrm{~b}$ ). Transannular displacement of bromide gives the oxonium ion $9 \mathbf{b}$, which on C-7 opening with chloride gives the notoryne diastereomer $\mathbf{3 b}$, (3Z)-laurendecumenyne B. Displacement of bromide by water (or a water equivalent) ${ }^{27}$ gives diastereomer $\mathbf{2 b}$ of the chloroenyne from L. majuscula. The laurediols exist naturally as unequal mixtures of $(R, R),(S, S),(3 E),(3 Z)$, $(12 E)$, and (12Z)-diastereomers. ${ }^{25}$ The $(3 E)$ and (12Z)laurediols $\mathbf{4 c}$ and $\mathbf{4 d}$ are therefore also plausible starting points for the biosynthesis of the chloroenyne 2 , leading to stereostructures 2c and 2d (Figure 1c). ${ }^{29}$ Based on our biosynthetic analysis, the structure of the chloroenyne from $L$. majuscula is plausibly, therefore, one of the four diastereomers $2 a, 2 b, 2 c$, or $2 d$, each of which could be produced from the above biogenetic schemes. ${ }^{30}$ We then used quantum chemistry to predict the most likely stereostructure for 2 , based on computed ${ }^{13} \mathrm{C}$ and ${ }^{1} \mathrm{H}$ chemical shifts for each of the 32 possible diastereomers. ${ }^{31}$ 
(a)<smiles>C#CC=CCC(O)C(O)CC=CCC=CCCC</smiles><smiles>C#C/C=C/CC(O)C(O)C/C=C/C/C=C/C1[B]C1CC</smiles><smiles>C#C/C=C/CC(O)C1(O)C/C=C\CC(Br)C(CC)O1</smiles>

$5 a$

$6 a$<smiles>C#CC=CCC(O)C1(O)CC2[Bi]C(CC1Br)C2CC</smiles><smiles>CC#C/C=C/C[C@@H]1C[C@@H](Cl)[C@H]([C@H]2C[C@H](Br)[C@@H](CO)O2)O1</smiles>

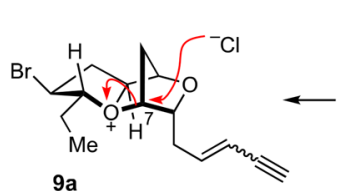

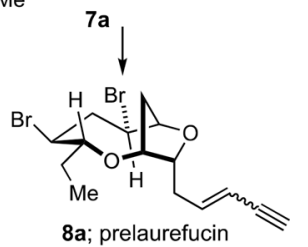

(b)<smiles>C#CC=CCC(O)C(O)CC=CCC=CCC</smiles>

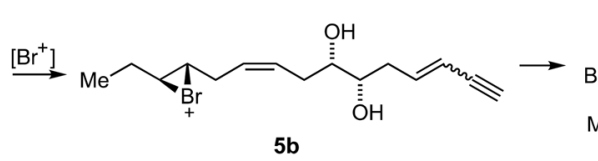<smiles>C#C/C=C/C[C@@H](O)[C@@H]1CC=CC[C@H](Br)[C@H](CO)O1</smiles><smiles>C#C/C=C/C[C@@H](O)C1CC2O[C@@H](CC)C(Br)C[C@H]2CC1Br</smiles>

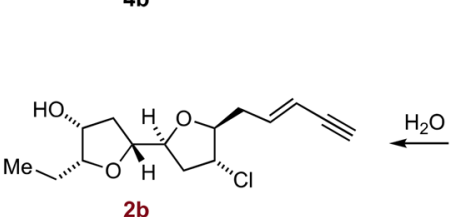<smiles>C#C/C=C/C[C@H]1O[C@@H](C(C)O)CC1Cl</smiles>

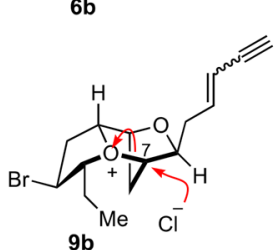

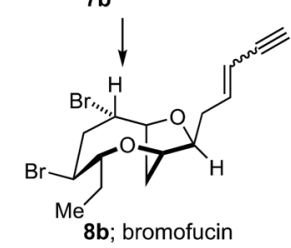

(c)

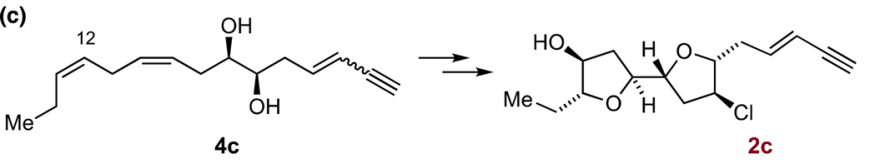<smiles>C#CC#CC=CC[C@@H](O)[C@@H](O)CC=CCC=CCC</smiles><smiles>C#C/C=C/CC1OC2CC(Cl)C(CC2O)O1</smiles>

Figure 1. (a) Plausible biosynthesis of notoryne 3a as proposed by Suzuki and by Fukuzawa and Murai along with the proposed biosynthesis of diastereomer $\mathbf{2 a}$ of the chloroenyne from L. majuscula. (b) Proposed biosynthesis of diastereomer $\mathbf{2} \mathbf{b}$ of the chloroenyne from $L$. majuscula via the natural products bromofucin $\mathbf{8 b}$ and laurendecumeyne B $\mathbf{3 b}$. (c) Proposed stereostructures $\mathbf{2 c}$ and $\mathbf{2 d}$ of the chloroenyne from $L$. majuscula derived from the (12Z)-laurediols $4 \mathrm{c}$ and $\mathbf{4 d}$.

\section{RESULTS AND DISCUSSION}

Computational Prediction. The specific challenges associated with the computational structural assignment of chloroenyne 2 (and related molecules) influenced the computational methods employed. First, each diastereomer is highly flexible. A Monte Carlo conformational search with the Merck Molecular Force Field (MMFF) was used to obtain the low-energy conformations within $10 \mathrm{~kJ} / \mathrm{mol}$ of the lowestenergy structure of each diastereomer. ${ }^{32}$ Across all of the diastereomers, there are 1277 conformers in this energy range that contribute to the predicted Boltzmann-weighted chemical shifts! Furthermore, the level of theory used for geometry optimization influences both the estimated populations and the computed chemical shifts of each conformation. Although MMFF geometries have been used in structure prediction, ${ }^{31 c, 33}$ DFT optimization allows for more confident structural assignment as there is a narrower distribution of errors with respect to experimental chemical shifts. ${ }^{16 a, 31 c}$ As an illustration, for 82 molecules with 709 experimentally assigned ${ }^{13} \mathrm{C}$ chemical shifts, we verified that DFT optimizations led to a $2 \mathrm{ppm}$ reduction in root-mean-square deviation ( $\mathrm{rmsd}$ ) compared to MMFF geometries, even though both sets of calculations used the same level of theory for shielding tensor calculation and empirical scaling (Figure S1). In this work, we optimized all structures with dispersion-corrected DFT, at the wB97XD/6-31G(d) level of theory ${ }^{34}$ with CPCM (Conductorlike Polarizable Continuum Model) chloroform. ${ }^{35}$ Conformer relative energies were checked against COSMO-DLPNO$\operatorname{CCSD}(\mathrm{T}) / \mathrm{cc}-\mathrm{pVTZ}$ single-point energies ${ }^{36}$ for one diastereomer and showed a good level of agreement $\left(R^{2}=0.90, \mathrm{rmsd}\right.$
$=3.1 \mathrm{~kJ} / \mathrm{mol}$ ) against this high accuracy method (Figure S2). Manual data processing is prohibitively difficult for so many conformations; a Python program was developed to automate all analysis given a collection of output files and a text file with experimental chemical shifts. Conformational Boltzmann weighting, empirical scaling, symmetry averaging, consideration of alternative assignments, and calculation of rmsd/MAD and DP4 for all structures are fully automated (Supporting Information shows example usage). We note that the DP4 workflow has now been automated (pyDP4) by Ermanis and Goodman. $^{12}$

Halogenated natural products pose a further challenge due to the so-called heavy-atom light-atom (HALA) effect. ${ }^{37}$ Relativistic spin-orbit contributions shift carbon atoms bonded to $\mathrm{Cl}, \mathrm{Br}$, or I to lower parts per million. In this work, we used the CHESHIRE database test set of molecules developed by Rablen, Bally, and Tantillo ${ }^{13}$ to derive new, optimal scaling parameters for mPW1PW91/6-311G(d,p)// wB97XD/6-31G(d) GIAO shielding tensors for ${ }^{13} \mathrm{C}$ and ${ }^{1} \mathrm{H}$ nuclei (Figure S3). Several chlorine-containing compounds appear in this data set, from which we found an additive correction of $7.6 \mathrm{ppm}$ applied to the shielding tensors of $\mathrm{C}-\mathrm{Cl}$ atoms results in an rmsd no worse than if these atoms are excluded entirely. Such a correction (which is level of theory dependent) has also been used by Rzepa and Braddock. ${ }^{38}$

We analyzed the Boltzmann-weighted ${ }^{13} \mathrm{C}$ and ${ }^{1} \mathrm{H}$ chemical shifts for all 32 diastereomers at every position except the hydroxyl proton. The variability in computed shifts across the entire set of diastereomers (Figure 2) shows the extent to which each nucleus acts as a reporter for stereochemical changes. Larger standard deviations are obtained for positions 


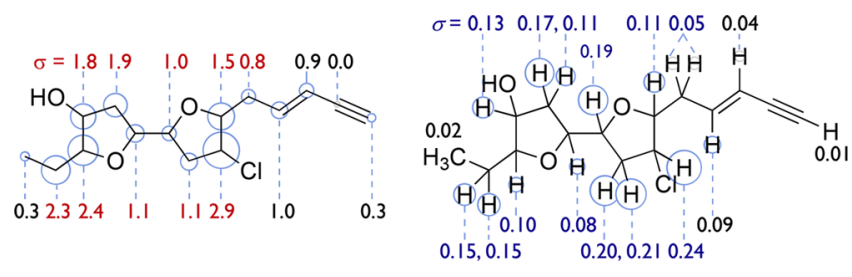

Figure 2. Standard deviations of computed ${ }^{13} \mathrm{C}$ and ${ }^{1} \mathrm{H}$ chemical shifts at each position over all 32 diastereomers of 2 . These values, illustrated by circle size, show the sensitivity of each shift to stereochemical changes. Values in black were excluded from experimental comparison.

which are inherently more sensitive to their relative stereochemistry. Stereostructure assignment can be made more confidently when these values are large in relation to the inherent accuracy of the computed chemical shifts versus experiment (i.e., by ensuring a higher signal-to-noise ratio).

Based on the work of Smith and Goodman, ${ }^{11}$ standard deviations of 2.3 and $0.19 \mathrm{ppm}$ for errors in ${ }^{13} \mathrm{C}$ and ${ }^{1} \mathrm{H}$ chemical shifts are representative of the underlying computational accuracy, although because previous calculations used MM geometries, these values are likely to be pessimistic for the DFT optimizations used here. For chloroenyne 2, a handful of nuclei have low variability and are thus very poor reporters of stereochemical information. Consistent with chemical intuition, these are exocyclic positions remote from stereocenters. These nuclei were excluded from further analysis as they contribute minimally to stereochemical assignment. The inclusion of the halogenated carbon atom is important as this is the best stereochemical reporter from all of the ${ }^{13} \mathrm{C}$ shifts. This is important because halogenated carbons have been omitted previously from structural prediction due to the aforementioned challenges associated with the HALA. ${ }^{15 \mathrm{c}}$ The ethyl ${ }^{13} \mathrm{CH}_{2}$ also provides one of the more diagnostic values. There are several protons which show a standard deviation larger than $0.19 \mathrm{ppm}$. This is consistent with the idea that structural differences in ${ }^{1} \mathrm{H}$ chemical shifts are more diagnostic than ${ }^{13} \mathrm{C}$ in relation to the underlying theoretical accuracy. ${ }^{39}$ We compared predicted chemical shifts for each diastereomer against those of the natural product. The ${ }^{13} \mathrm{C}$ spectrum was fully assigned, whereas for three pairs of protons, the best possible (lowest rmsd) assignment was generated automatically for each structure. The rmsd values and DP4 metrics were generated using $10{ }^{13} \mathrm{C}$ and $14{ }^{1} \mathrm{H}$ chemical shifts (Figure 3 ). If one assumes that the underlying error distribution of $n$ chemical shifts is Gaussian (in its original formulation, the DP4 metric assumes a $t$-distribution, although a Gaussian distribution was also proposed), the sum of squared errors obeys a $\chi^{2}$-distribution with $n$ degrees of freedom. The rmsd values can therefore be interpreted in a probabilistic fashion as is done for DP4: for example, incorrect structures can be rejected at the $p=0.05$ significance level, where the rmsd falls above a critical value (Figure 3). Importantly, the statistical significance of differences between rmsd values is intrinsically linked to the number of chemical shifts being compared. Although rmsd, MAE, and $R^{2}$ measures have been used previously to compare the relative likelihood of structures being correct, the statistical confidence of these measures has not received much attention. Here, in addition to DP4 values, we show the $95 \%$ confidence intervals for rmsd values, above which structures are deemed to be unlikely candidates.

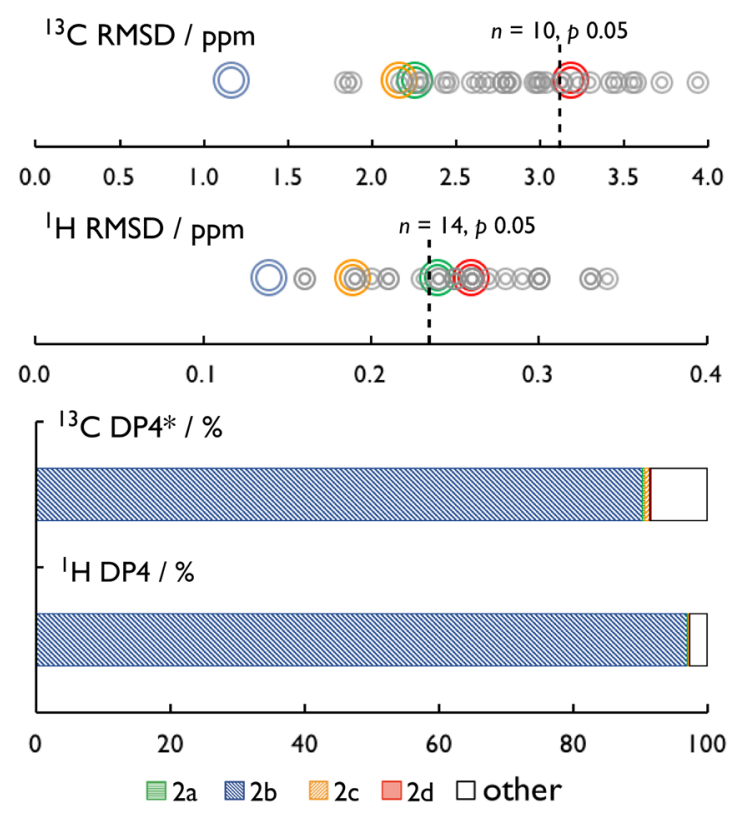

Figure 3. Comparison of computed ${ }^{13} \mathrm{C}$ and ${ }^{1} \mathrm{H}$ chemical shifts for 32 diastereomers of 2 against the natural product. The smallest rmsd values and largest DP4 probabilities point to biosynthetically predicted compound $\mathbf{2} \mathbf{b}$.

We found that one of the four biogenetically plausible structures (2b) was the best match for ${ }^{13} \mathrm{C}$ and ${ }^{1} \mathrm{H}$ experimental data, giving the smallest rmsd and largest DP4* values. The convergence of all four metrics, combined with biosynthetic arguments, overwhelmingly suggests the identity of the chloroenyne from L. majuscula as $\mathbf{2 b}$. In using the original $t$-distribution parameters $(\sigma, v)$ derived for MMFFoptimized geometries, we are being deliberately conservative: we expect a narrower error distribution using DFT optimizations that would penalize the less likely structures more severely. DP4 relies on individually scaling each structure against experiment, which can lead to a fortuitous improvement of incorrect structures, particularly for a small number of nuclei. Indeed, we found better predictive performance without individually scaling the ${ }^{13} \mathrm{C}$ shifts (indicated as DP4*).

All of our biosynthetic and computational analysis provided compelling evidence that the correct structure for the chloroenyne from L. majuscula was represented by $\mathbf{2 b}$; however, proof of this could only come through total synthesis. ${ }^{1,2 b}$ Additionally, we decided to synthesize all four biosynthetically relevant diastereomers of the chloroenyne from L. majuscula, which would allow us to obtain NMR data for each diastereomer. With these data in hand, we could further test the computational methods; would it be possible computationally to correctly identify each of the four biosynthetically predicted diastereomers $(\mathbf{2 a}-\mathbf{d})$ from the computed data of the 32 possible diastereomers of the chloroenyne from L. majuscula?

Synthesis. The proposed synthesis of the four biomimetically plausible diastereomers of the chloroenyne form $L$. majuscula $(\mathbf{2 a}-\mathbf{d})$ presented us with the opportunity to modify and improve our modular route to $2,2^{\prime}$-bifuranyl natural products. ${ }^{15 \mathrm{~d}}$ The retrosynthetic synthetic route to diastereomers $\mathbf{2 a}$ and $\mathbf{2 c}$ is shown in Figure 4. Enynes $\mathbf{2 a}$ and $\mathbf{2 c}$ were to be readily derived from the $2,2^{\prime}$-bifuranyl 10 , which itself was to be derived from 11 and subsequently from diol 12 following a route analogous to that described in our recent 


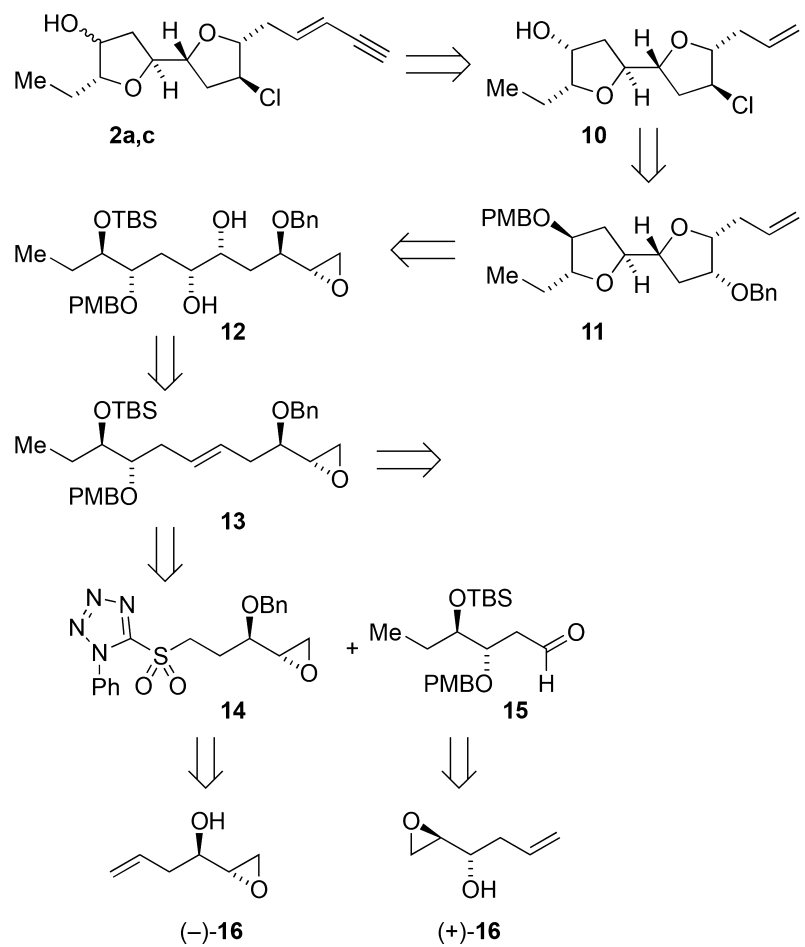

Figure 4. Retrosynthetic analysis of diastereomers $\mathbf{2 a}$ and $\mathbf{2 c}$.

synthesis of elatenyne. ${ }^{15 \mathrm{~d}}$ Diol $\mathbf{1 2}$ was to be derived from alkene 13, which was to be constructed by Julia-Kocienski olefination of aldehyde 15 with sulfone $14 .{ }^{40}$ The two coupling partners 14 and 15 were to be derived from protection of the known enantiomeric epoxy alkenes (+)-16 and (-)-16. In the forward direction (Scheme 1), the known benzyl-protected epoxy alkene 17, prepared according to the method of Crimmins, ${ }^{41}$ was converted into alcohol $\mathbf{1 8}$ by ozonolysis with in situ reduction $\left(\mathrm{PPh}_{3}\right.$ then $\left.\mathrm{NaBH}_{4}\right)$. Alcohol 18 was then converted into the corresponding tetrazole sulfone 14 by Mitsunobu reaction followed by oxidation. ${ }^{40}$ Aldehyde $\mathbf{1 5}$ was readily prepared from alcohol (+)-16, which on PMB protection gave ether $19 .^{15 \mathrm{~d}}$ Copper-catalyzed ring opening of epoxide 19 with methylmagnesium bromide ${ }^{41}$ followed by silyl protection gave alkene 20. Ozonolysis of alkene $\mathbf{2 0}$ and reductive phosphine workup gave the required aldehyde $\mathbf{1 5}$. Julia-Kocienski ${ }^{40,42}$ coupling of sulfone tetrazole $\mathbf{1 4}$ with aldehyde 15 proceeded in good yield with >20:1 E/Zselectivity to give alkene $13 .^{43}$ Sharpless asymmetric dihydroxylation $^{44}$ of 13 using super-AD-mix $\beta^{45}$ gave the corresponding diols in $91 \%$ yield as a $6: 1$ mixture of syndiastereomers; diol 12 could be isolated in pure form in 64\% yield. Under acid catalysis, diol 12 underwent cyclization to give THF 21. Formation of the second THF ring was achieved using a three-step procedure. Exposure of diol 21 to mesyl chloride and Hünig's base gave dimesylate 23 which, without purification, was treated with $( \pm)$-10-camphorsulfonic acid to remove the silyl-protecting group. Exposure of the resulting alcohol to potassium tert-butoxide gave 2,2'-bifuranyl 24 in $52 \%$ yield over three steps.

During optimization studies, the monomesylate 22 was isolated and characterized. Mosher ester formation using 22 allowed the sense of the Sharpless asymmetric dihydroxylation reaction to be confirmed. ${ }^{46}$ As with the synthesis of elatenyne, ${ }^{15 \mathrm{~d}}$ forcing conditions $\left(\mathrm{Bu}_{4} \mathrm{NI}\right.$, toluene, reflux $)$ were

\section{Scheme 1. Synthesis of Chloride $28^{a}$}

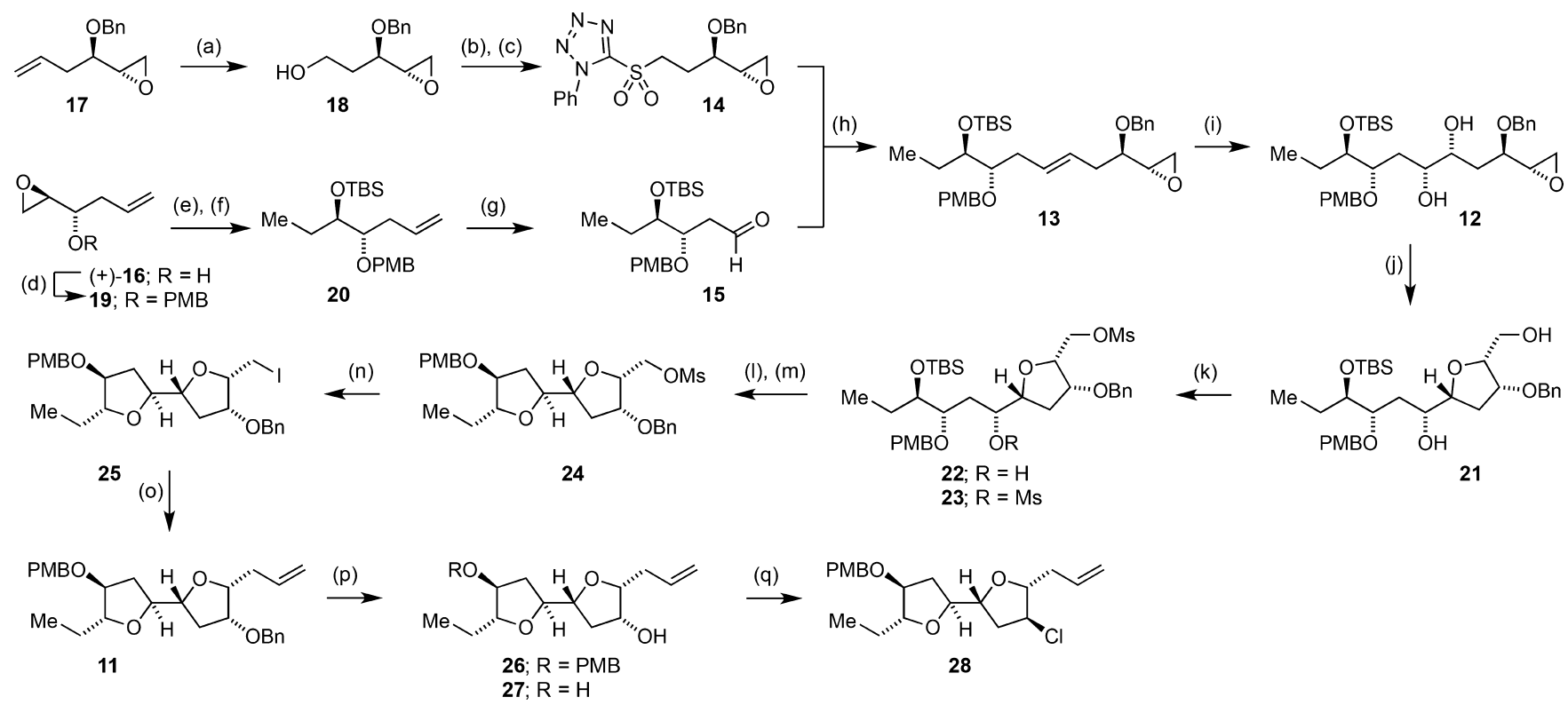

${ }^{a}$ Reagents and conditions: (a) $\mathrm{O}_{3} / \mathrm{O}_{2}, \mathrm{CH}_{2} \mathrm{Cl}_{2}, \mathrm{MeOH},-78{ }^{\circ} \mathrm{C}$, then $\mathrm{PPh}_{3}, 30 \mathrm{~min}$, then $\mathrm{NaBH}_{4},-78{ }^{\circ} \mathrm{C}$ to rt, $2 \mathrm{~h}, 92 \%$; (b) DIAD, $\mathrm{PPh}$, THF, 0 ${ }^{\circ} \mathrm{C}$, then 18, then 1-phenyl-1H-tetrazole-5-thiol, rt, $16 \mathrm{~h}, 88 \%$; (c) 3-chloroperbenzoic acid, $\mathrm{CH}_{2} \mathrm{Cl}_{2}, \mathrm{rt}, 72 \mathrm{~h}, 60 \%$; (d) NaH, PMBBr, Bu ${ }_{4} \mathrm{NI}, \mathrm{THF}$, $-78{ }^{\circ} \mathrm{C}$ to rt, $16 \mathrm{~h}$; (e) $\mathrm{MeMgBr}, \mathrm{CuI}, \mathrm{THF},-20{ }^{\circ} \mathrm{C}, 1 \mathrm{~h}, 88 \%$ (two steps); (f) $\mathrm{TBSCl}$, imidazole, DMF, $60{ }^{\circ} \mathrm{C}, 16 \mathrm{~h}, 99 \%$; $(\mathrm{g}) \mathrm{O}_{3} / \mathrm{O}_{2}, \mathrm{CH}_{2} \mathrm{Cl}_{2}$, $-78{ }^{\circ} \mathrm{C}$, then $\mathrm{PPh}_{3},-78{ }^{\circ} \mathrm{C}$ to rt, $16 \mathrm{~h}, 98 \%$; (h) 14, DME, NaHMDS, $-78{ }^{\circ} \mathrm{C}, 15 \mathrm{~min}$, then add 15 in DME, $-78{ }^{\circ} \mathrm{C}, 1 \mathrm{~h}$, then warm to rt, $2 \mathrm{~h}$, $80 \%$; (i) $\mathrm{K}_{2} \mathrm{OsO}_{4}(\mathrm{OH})_{4}, \mathrm{~K}_{3} \mathrm{Fe}(\mathrm{CN})_{6}$, (DHQD) ${ }_{2} \mathrm{PHAL}, \mathrm{K}_{2} \mathrm{CO}_{3}, \mathrm{MeSO}_{2} \mathrm{NH}_{2}, t \mathrm{BuOH}$, water, rt, $24 \mathrm{~h}, 64 \%$ pure 12 (6:1 mixture of syn-diol diastereomers $91 \%$ total); (j) ( \pm )-10-camphorsulfonic acid, $\mathrm{CH}_{2} \mathrm{Cl}_{2}, 0{ }^{\circ} \mathrm{C}, 2 \mathrm{~h}, 86 \%$; (k) $\mathrm{MsCl}, i-\mathrm{Pr}_{2} \mathrm{NEt}, \mathrm{CH}_{2} \mathrm{Cl}_{2}, 0{ }^{\circ} \mathrm{C}, 1 \mathrm{~h}$; $(\mathrm{l})( \pm)-10$ camphorsulfonic acid, $\mathrm{CH}_{2} \mathrm{Cl}_{2}, \mathrm{MeOH}, \mathrm{rt}, 24 \mathrm{~h}$; (m) $t$-BuOK, $t \mathrm{BuOH}, 35{ }^{\circ} \mathrm{C}, 52 \%$ (three steps); (n) $\mathrm{Bu}_{4} \mathrm{NI}$, toluene, $110{ }^{\circ} \mathrm{C}, 16 \mathrm{~h}, 80 \%$; $(\mathrm{o})$ $\mathrm{CH}_{2}=\mathrm{CHMgBr}$, benzene, THF, $40{ }^{\circ} \mathrm{C}, 3 \mathrm{~h}, 57 \%$; (p) Li, 4,4'-di-tert-butyl-1,1'-biphenyl, THF, $-78{ }^{\circ} \mathrm{C}, 77 \%$; (q) $\mathrm{CCl}_{4}, \mathrm{PPh}_{3}, \mathrm{CH}_{2} \mathrm{Cl}_{2}, \mathrm{rt}^{\prime}, 3 \mathrm{~h}, 78 \%$. 
required to convert the mesylate $\mathbf{2 4}$ into the corresponding iodide 25. Iodide 25 underwent displacement with vinylmagnesium bromide in a mixed benzene/THF solvent system to give the allyl-substituted 2,2'-bifuranyl 11 in $57 \%$ yield. ${ }^{47}$ Deprotection of the benzyl group in $\mathbf{1 1}$ in the presence of the PMB group was achieved by titrating $\mathrm{LiDBB}^{48}$ into a THF solution of 11 to minimize formation of diol 27. Attempted chlorination of alcohol 26 using triphenylphosphine with carbon tetrachloride as both reagent and solvent led to very little conversion of $\mathbf{2 6}$ into chloride 28 . $^{49}$ Appel reported a large solvent effect on the chlorination of alcohols using tertiary phosphines and carbon tetrachloride with the use of dichloromethane and acetonitrile, resulting in significant rate enhancements. $^{49}$ Conducting the chlorination of alcohol 26 using dichloromethane as solvent gave chloride $\mathbf{2 8}$ in $\mathbf{7 8 \%}$ yield. Conversion of chloride $\mathbf{2 8}$ into the biosynthetically plausible diastereomers $2 \mathrm{a}$ and $2 \mathrm{c}$ required introduction of the (E)-enyne (Scheme 2). Previously the Oxford group have used

Scheme 2. Synthesis of Diastereomers $2 a$ and $2 c^{a}$
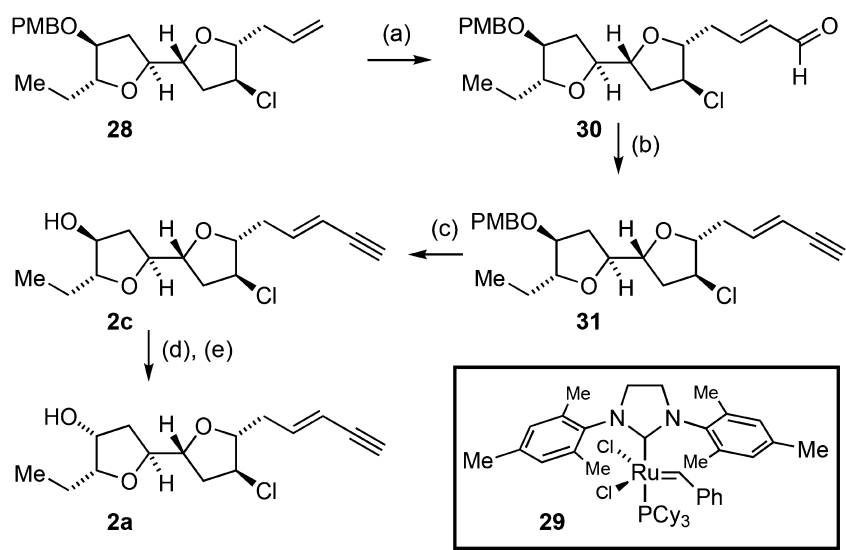

${ }^{a}$ Reagents and conditions: (a) crotonaldehyde, catalyst $29, \mathrm{CH}_{2} \mathrm{Cl}_{2}$, $40{ }^{\circ} \mathrm{C}$, $1 \mathrm{~h}$ then $\mathrm{Me}_{2} \mathrm{SO}$, rt, $16 \mathrm{~h}, 88 \%$; (b) (trimethylsilyl)diazomethane, $n$-BuLi, THF, add $30,-78{ }^{\circ} \mathrm{C}$ to rt, $1 \mathrm{~h} 70 \%$; (c) $\mathrm{BCl}_{3}$. $\mathrm{SMe}_{2}, \mathrm{CH}_{2} \mathrm{Cl}_{2}, \mathrm{rt}, 5 \mathrm{~min}, 84 \%$; (d) DIAD, $\mathrm{PPh}_{3}$, THF, $0{ }^{\circ} \mathrm{C}$, then $2 \mathrm{c}$, then 4-nitrobenzoic acid, rt; (e) $\mathrm{K}_{2} \mathrm{CO}_{3}, \mathrm{MeOH}, \mathrm{rt}, 20 \mathrm{~min}, 25 \%$ two steps).

a Wittig reaction with the ylide derived from (3-trimethylsilyl2-propynyl)triphenylphosphonium bromide ${ }^{15 a, b, d}$ for the stereoselective synthesis of $(E)$-enynes from aldehydes; however, the diastereocontrol in these reactions was never above 9:1 E/ $Z$. We therefore elected to use the recently developed methodology for $(E)$-enyne synthesis from alkenes reported by the Seoul group, which gives very high $E$-selectivity. ${ }^{15 \mathrm{~d}, 16 a, 50}$ Cross-metathesis of alkene $\mathbf{2 8}$ with crotonaldehyde and Grubbs' second generation catalyst 29 gave the corresponding $\alpha, \beta$-unsaturated aldehyde 30 , which was immediately exposed to lithiated trimethylsilyl diazomethane (Colvin-Ohira homologation) to give enyne 31 . Removal of the para-methoxy benzyl group ${ }^{51}$ from 31 gave $2 c$, the first of the enyne targets. Mitsunobu inversion ${ }^{52}$ of the secondary alcohol in $2 \mathrm{c}$ followed by ester methanolysis gave $2 \mathrm{a}$, the second of the biomimetically plausible diastereomers.

The synthesis of the final two biosynthetically plausible diastereomers of the chloroenyne from L. majuscula (enynes $\mathbf{2 b}$ and 2d) began from the known 2,2'-bifuranyl 32, an intermediate in our recent synthesis of elatenyne (Scheme $3) .{ }^{15 d}$ Selective removal of the para-bromobenzyl group in the
Scheme 3. Synthesis of Diastereomers $2 b$ and $2 d^{a}$<smiles></smiles>

$\stackrel{\text { (a) }}{\longrightarrow}$

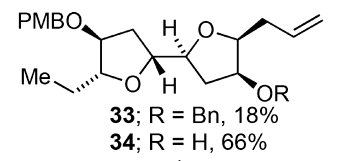

(b)<smiles>C=CCC1OC([C@H]2CC(O)[C@@H](CO)O2)CC1Cl</smiles>

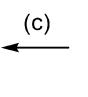<smiles>[2H][C@]1(C2CC(CO)C(CC=C)O2)CC(Cl)C(CC=C)C1</smiles>

35<smiles>C=CCC1OC(C2CC(O)C(CO)O2)CC1Cl</smiles><smiles>C1CCCCC1</smiles><smiles>CC[C@H]1O[C@H](C2CC(Cl)C(C/C=C/C=O)O2)CC1O</smiles>
(g), (h) $\downarrow^{38}$<smiles>C#C/C=C/CC1OC([C@H]2C[C@H](O)[C@@H](CC)O2)CC1Cl</smiles>

${ }^{a}$ Reagents and conditions: (a) Li, 4,4'-di-tert-butyl-1,1'-biphenyl, bis (2-methoxyethyl)amine, THF, $-78{ }^{\circ} \mathrm{C}, 18 \%$ of $33,66 \%$ of 34 ; (b) $\mathrm{CCl}_{4}, \mathrm{PPh}_{3}, \mathrm{CH}_{2} \mathrm{Cl}_{2}, \mathrm{rt}, 3 \mathrm{~h}, 96 \%$; (c) $\mathrm{BCl}_{3} \cdot \mathrm{SMe}_{2}, \mathrm{CH}_{2} \mathrm{Cl}_{2}, \mathrm{rt}, 5 \mathrm{~min}$; 94\%; (d) DIAD, $\mathrm{PPh}_{3}$, THF, $0{ }^{\circ} \mathrm{C}$, then 36, then 4-nitrobenzoic acid, rt; (e) $\mathrm{K}_{2} \mathrm{CO}_{3}, \mathrm{MeOH}, \mathrm{rt}, 30 \mathrm{~min}, 79 \%$ (two steps); (f) crotonaldehyde, catalyst $29, \mathrm{CH}_{2} \mathrm{Cl}_{2}, 40{ }^{\circ} \mathrm{C}, 90 \mathrm{~min}$; (g) (trimethylsilyl)diazomethane, $n$-BuLi, THF, add $37,-78$ to $0{ }^{\circ} \mathrm{C}$; (h) $\mathrm{Bu}_{4} \mathrm{NF}$, THF, $0{ }^{\circ} \mathrm{C}, 5$ min, $39 \%$ (three steps); (i) DIAD, $\mathrm{PPh}_{3}$, THF, $0{ }^{\circ} \mathrm{C}$, then $\mathbf{2 b}$, then 4-nitrobenzoic acid, rt; $(\mathrm{j}) \mathrm{K}_{2} \mathrm{CO}_{3}, \mathrm{MeOH}$, rt, $30 \mathrm{~min}$.

presence of the more electron-rich para-methoxy benzyl group was achieved using $\mathrm{LiDBB}^{48}$ in the presence of a proton donor, $^{53}$ which gave the requisite alcohol 34 in $66 \%$ yield along with $18 \%$ of the benzyl ether $33 .{ }^{54}$ Chlorination of 34 as before $^{49}$ provided chloride 35 in $96 \%$ yield, which was readily deprotected under Lewis acidic conditions to give alcohol 36. ${ }^{51}$ Mitsunobu inversion of $36^{52}$ to give 37 , followed by enyne introduction, as before, ${ }^{15 \mathrm{~d}, 50}$ gave the third biomimetic diastereomer $\mathbf{2 b}$. A further Mitsunobu reaction gave the fourth and final biomimetic diastereomer $\mathbf{2 d}{ }^{55}$

Analysis. Having completed the total synthesis of all four of the biosynthetically relevant diastereomers of the chloroenyne from L. majuscula $(\mathbf{2 a - d})$, we were delighted to find that the ${ }^{1} \mathrm{H}$ and ${ }^{13} \mathrm{C}$ NMR data for diastereomer $2 b$ were in excellent agreement with that reported for the natural product. ${ }^{17}$ Our synthesis of the chloroenyne from $L$. majuscula $\mathbf{2} \mathbf{b}$ proceeds in 16 steps (longest linear sequence) from (+)-16. The optical rotation for our synthetic $\mathbf{2} \mathbf{b}$ was in good agreement in terms of both sign and magnitude with that recorded for the natural product, ${ }^{17}$ demonstrating that the absolute configuration of the chloroenyne from L. majuscula is as represented by $\mathbf{2 b}$. The chloroenyne from $L$. majuscula $\mathbf{2 b}$ thus sits on the same proposed biosynthetic pathway as elatenyne, ${ }^{15 \mathrm{~d}}$ laurendecumenyne $\mathrm{B},{ }^{15 \mathrm{~d}}$ and laurefurenynes $\mathrm{A}$ and $\mathrm{B},{ }^{16}$ proceeding from $(3 E / Z)$-laurediols $\mathbf{4} \mathbf{b}$ via the bromofucins $\mathbf{8 b}$.

The identification of the full stereostructure of the chloroenyne from L. majuscula as $\mathbf{2} \mathbf{b}$ on the basis of DFT methods demonstrates the power and utility of these methods to aid in the structure determination of stereochemically rich organic molecules. The synthesis of the remaining three biosynthetically relevant diastereomers $\mathbf{2 a}, \mathbf{2 c}$, and $\mathbf{2 d}$ provided us with the opportunity to further test the computational 
vs. experimental $2 a$
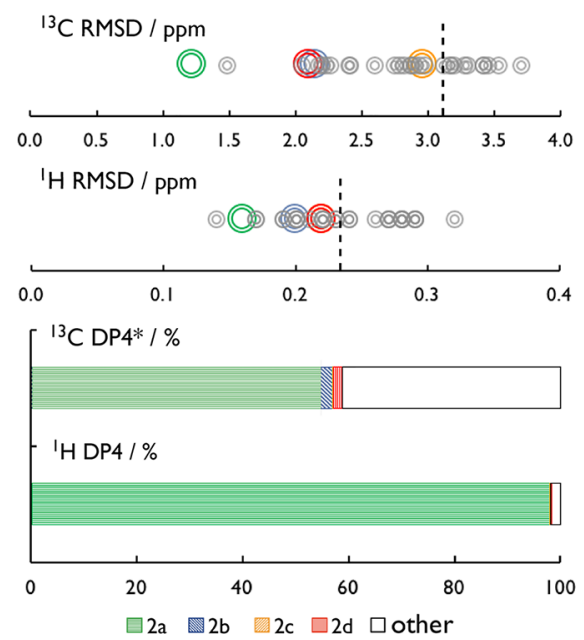

vs. experimental $2 c$
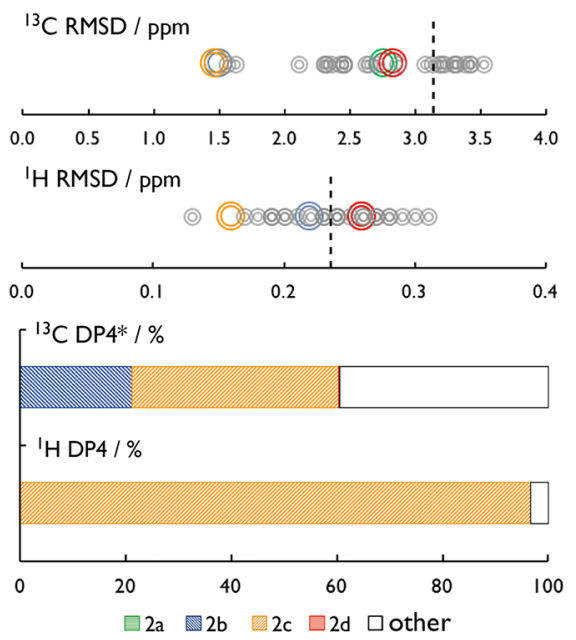

vs. experimental 2d
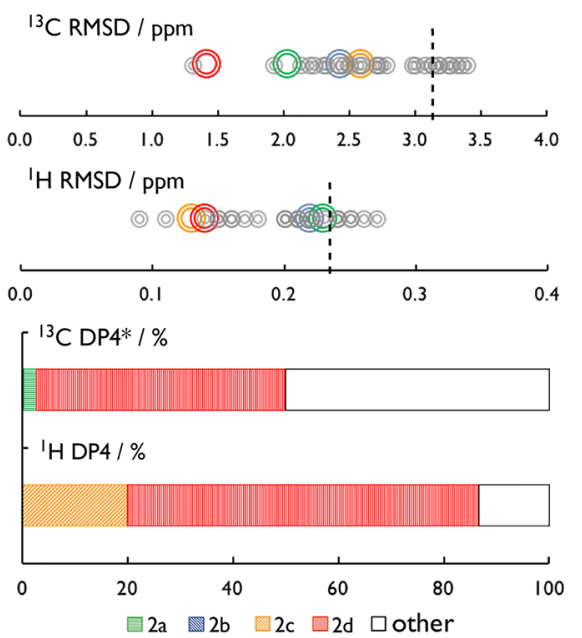

Figure 5. Comparison of computed ${ }^{13} \mathrm{C}$ and ${ }^{1} \mathrm{H}$ chemical shifts for 32 diastereomers of $\mathbf{2}$ against experimental spectra for $\mathbf{2 a}$, $\mathbf{2 c}$, and $\mathbf{2 d}$.

Scheme 4. Key Step in the Seoul Synthesis of Laurefucin, along with the Proposed Route to Notoryne

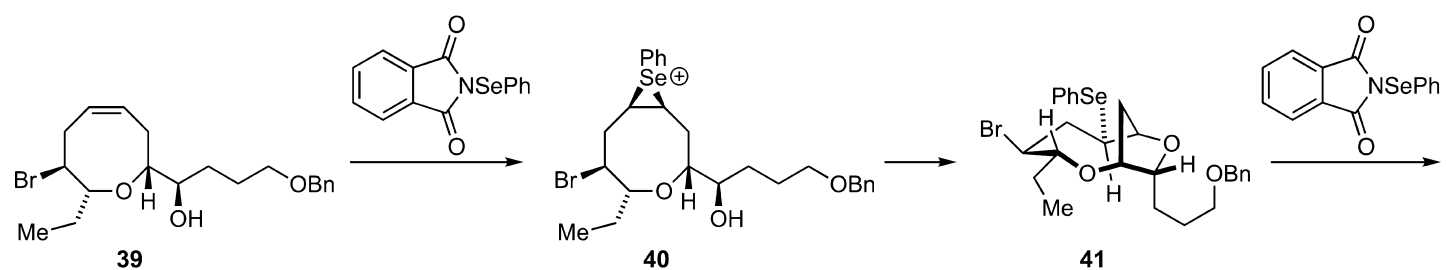

39<smiles>C#C/C=C\C[C@H]1O[C@@H](C2CC(Br)[C@@H](CO)O2)CC1Cl</smiles>

(Z)-3a; notoryne
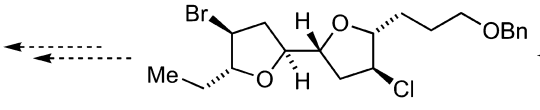

46
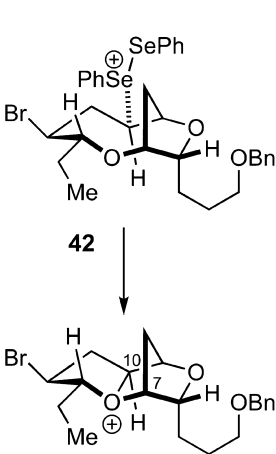

43 water

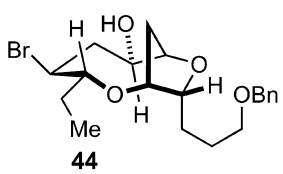

methods. We had acquired ${ }^{1} \mathrm{H}$ and ${ }^{13} \mathrm{C}$ NMR data for the chloroenynes 2a, 2c, and $\mathbf{2 d}$, which allowed us to determine whether it would be possible, computationally and correctly, to identify each of these diastereomers from the computed data of the 32 possible diastereomers of the chloroenyne from $L$. majuscula. We found the correct stereostructures identified among structures with the smallest rmsd values: the two structures with the lowest rmsd values contain the correct structure in all but one case $\left({ }^{1} \mathrm{H}\right.$ of $\left.\mathbf{2 d}\right)$, where it is in the lowest four (Figure 5). ${ }^{1} \mathrm{H}$ DP4 values were more diagnostic, with the correct structure predicted with $60-98 \%$ confidence (compared to $40-55 \%$ for ${ }^{13} \mathrm{C}$ ). The product of the DP4/ DP4* values for both nuclei provide an unequivocal and, importantly, correct stereostructure prediction for all four diastereomers studied, including the natural product.

Notoryne. Notoryne $(Z)-3 \mathrm{a}$ is the first halogenated $2,2^{\prime}$ bifuranly natural product isolated from Laurencia spp. ${ }^{56}$ The structure of $(Z)$-notoryne $(Z)$-3a was originally assigned by careful chemical degradation and comparison with chemical degradation products from laurefucin and laurencin, whose structures and absolute configurations were securely established through single-crystal X-ray analysis. ${ }^{21}$ As noted above, a biosynthesis of notoryne 3 was first proposed by Suzuki and by Fukuzawa and Murai (Figure 1a). ${ }^{21,24}$ Previously, the Oxford group had prepared the 2,2'-bifuranyl 28 (Scheme 1), with the necessary stereochemical arrangement for ready conversion into notoryne ( $Z$ )-3a. Additionally, the Seoul group had demonstrated a number of biomimetic syntheses of halogenated natural products from Laurencia spp., including the synthesis of the 2,2'-bifuranyl natural products $(Z)$ - and $(E)$ elatenyne $^{15 \mathrm{~d}}$ and laurendecumenyne $B{ }^{15 \mathrm{~d}}$ In their synthesis of laurefucin, ${ }^{50 a}$ the Seoul group had prepared the bromooxocene 39, which on treatment with $N$-phenylselenophthalimide $(N$ PSP) under aqueous acidic conditions gave rise to the [5.2.1] dioxabicyclic bromide 44 in quantitative yield (Scheme 4); the bromide was readily converted into the natural product laurefucin 45. The formation of the [5.2.1]dioxabicyclic bromide 44 most likely follows the mechanism indicated. Here, seleniranium ion formation occurs from the oxocene 39, giving 40, which undergoes ether formation to yield the 
Scheme 5. Oxford and Seoul Syntheses of Notoryne ${ }^{a}$

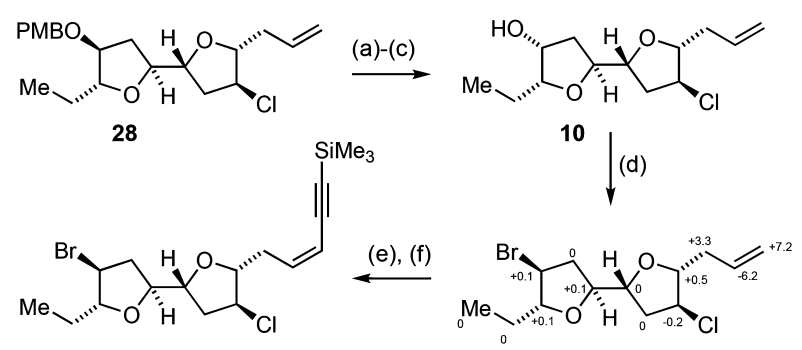

48<smiles>C/C=C\C[C@H]1O[C@]2(C[C@H](Br)[C@@H](CO)O2)C[C@H]1Cl</smiles>

(Z)-3a

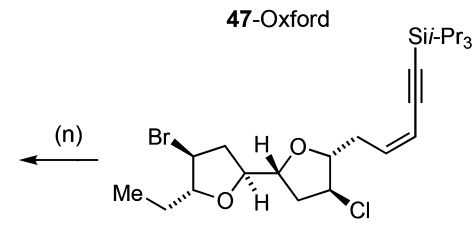

52

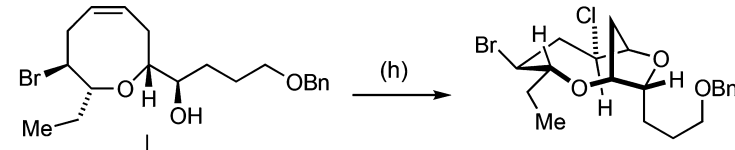

39 (i), (j)

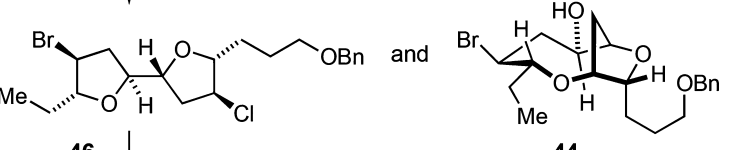

46 (k), (l)

(m)

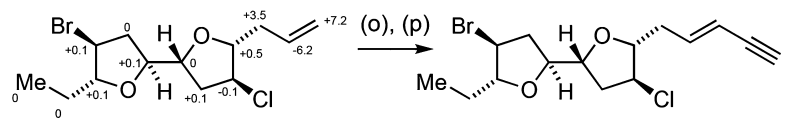

47-Seoul

$(E)-3 a$

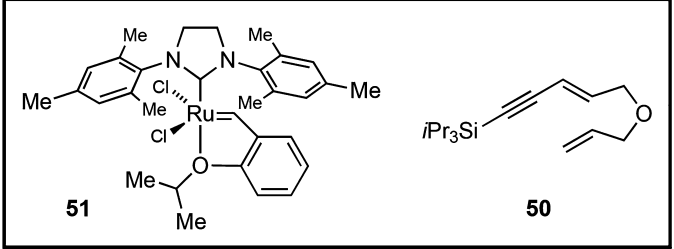

${ }^{a}$ Reagents and conditions: (a) $\mathrm{BCl}_{3} \cdot \mathrm{SMe}_{2}, \mathrm{CH}_{2} \mathrm{Cl}_{2}, \mathrm{rt}, 5 \mathrm{~min}, 95 \%$; (b) $\mathrm{DIAD}, \mathrm{PPh}_{3}, \mathrm{THF}, 0{ }^{\circ} \mathrm{C}$, then 4-nitrobenzoic acid, $\mathrm{rt}, 74 \%$; (c) $\mathrm{K}_{2} \mathrm{CO}_{3}$, $\mathrm{MeOH}, \mathrm{rt}, 20 \mathrm{~min}, 91 \%$; (d) $\mathrm{CBr}_{4}, \mathrm{PPh}_{3}$, toluene, $80{ }^{\circ} \mathrm{C}$, $75 \mathrm{~min}, 75 \%$; (e) $\mathrm{O}_{3}, \mathrm{CH}_{2} \mathrm{Cl}_{2},-78{ }^{\circ} \mathrm{C}$ then $\mathrm{PPh}_{3},-78{ }^{\circ} \mathrm{C}$ to rt, $15 \mathrm{~h}$; (f) $\mathrm{TMSC} \equiv$ CCH2TBS, tBuLi, THF, $-78{ }^{\circ} \mathrm{C}, 1 \mathrm{~h}$, then $\mathrm{Ti}(\mathrm{OiPr}), 10 \mathrm{~min}$, then add $47,-78{ }^{\circ} \mathrm{C}, 30 \mathrm{~min}, \mathrm{rt}, 30 \mathrm{~min}, 32 \%$ (two steps); (g) TBAF, THF, -20 ${ }^{\circ} \mathrm{C}$, 5 min, quant.; (h) PhSeCl, $n$-hexane; (i) PhSeCl (3 equiv), activated silica gel, $n$-hexane, rt, $72 \mathrm{~h}$; (j) $\mathrm{CH}_{3} \mathrm{CN} / \mathrm{H}_{2} \mathrm{O}(9: 1)$, rt, 24 h, 80\% of 46, $20 \%$ of 44; (k) $\mathrm{H}_{2}, \mathrm{Pd}(\mathrm{OH})_{2} / \mathrm{C}$, EtOH, $1 \mathrm{~h}, 95 \%$; (l) o-nitrophenylselenocyanide, (Oct) ${ }_{3} \mathrm{P}$, THF, rt, 10 min, then $\mathrm{H}_{2} \mathrm{O}_{2}, 0{ }^{\circ} \mathrm{C}$ to rt, $24 \mathrm{~h}, 85 \%$; $(\mathrm{m})$ 50, catalyst 51, benzene, $70{ }^{\circ} \mathrm{C}, 1.5 \mathrm{~h}$, then additional 50 and 51, 82\% 3:1 Z/E; (n) TBAF, THF, $0{ }^{\circ} \mathrm{C}, 1 \mathrm{~h}, 95 \%$; (o) crotonaldehyde, catalyst 29 , $\mathrm{CH}_{2} \mathrm{Cl}_{2}, 40^{\circ} \mathrm{C}, 1.5 \mathrm{~h}$ then $\mathrm{Me}_{2} \mathrm{SO}, \mathrm{rt}, 12 \mathrm{~h}$; (p) (trimethylsilyl)diazomethane, LDA, THF, -78 to $0{ }^{\circ} \mathrm{C}, 2 \mathrm{~h}, 88 \%$ (two steps). Note: The difference in the ${ }^{13} \mathrm{C} N M R$ chemical shifts between the synthetic compounds 47 prepared by the Oxford and Seoul groups and natural notoryne ( $Z$ )-3a are shown adjacent to the relevant carbon atoms in structures 47 .

selenide 41. Activation of the selenide group in 41 by reaction with further N-PSP gives prelaurefucin surrogate 42. Transannular $\mathrm{C}-\mathrm{O}$ bond formation then occurs, giving the key oxonium ion 43 . Attack at $\mathrm{C}-10$ by water with loss of a proton leads to the laurefucin precursor 44 that was readily transformed into the natural product 45. Opening of the oxonium ion 43 at $\mathrm{C}-7$ by chloride with inversion of configuration would yield the notoryne precursor 46 with the correct absolute configuration for synthesis of the natural product $(Z)-3 a$. Given the previous preparation of the 2,2'bifuranyl 28 and the oxocene 39 , we reasoned that synthesis of the natural product notoryne could be readily achieved by two independent routes (Scheme 5). The Oxford group began their synthesis from the previously prepared chloroalcohol 28, which was readily converted into chloroalcohol 10 through deprotection, Mitsunobu inversion, and saponification. Bromination of alcohol 10 using the Hooz procedure ${ }^{57}$ gave bromide 47. The Seoul team prepared the same bromide beginning with their previously prepared oxocene 39. After extensive experimentation, the Seoul team found that exposure of the oxocene alcohol 39 to phenylselenyl chloride in the presence of activated silica gel followed by treatment of the crude mixture with water in acetonitrile gave the 2,2'-bifuranyl chloride 46 along with alcohol $44 .^{58}$ It is of note that in the absence of silica gel, the [5.2.1]-bicyclic chloride 49 was formed. $^{50 a}$ In both the Oxford and Seoul syntheses, the chlorine-bearing carbon atoms could be identified using ${ }^{13} \mathrm{C}$ NMR chlorine-induced isotopic shift. ${ }^{59}$ The Seoul group converted the benzyl-protected alcohol 46 into the corresponding alkene 47 using standard procedures. Comparison of the ${ }^{13} \mathrm{C}$ NMR chemical shifts of the 2,2'-bifuranyls 47 synthesized in Oxford and Seoul, with the corresponding chemical shifts for notoryne, indicated that the synthesized material had the same stereostructure as that of the natural product (Scheme 5). Completion of the synthesis of the natural products was accomplished by two independent routes. In Oxford, the terminal alkene in $\mathbf{4 7}$ was subject to ozonolysis followed by reductive workup to give the corresponding aldehyde (uncharacterized) that was subject to a YamamotoPetersen reaction ${ }^{60}$ to give the $(Z)$-enyne 48 with high diastereoselectivity. Removal of the terminal silyl group was readily achieved on brief exposure of $\mathbf{4 8}$ to fluoride to give notoryne $(Z)$-3a. In Seoul, the $(Z)$-enyne was introduced directly from the terminal alkene 47 via a relay crossmetathesis using enyne $\mathbf{5 0}$ and catalyst $\mathbf{5 1},{ }^{15 \mathrm{~d}, 50 \mathrm{~b}, 61}$ which gave the desired enyne $\mathbf{5 2}$ as a 3:1 mixture of $Z / E$-enynes in $82 \%$ combined yield.

Fluoride treatment of $\mathbf{5 2}$ gave notoryne (Z)-3a. The Oxford and Seoul ${ }^{1} \mathrm{H}$ and ${ }^{13} \mathrm{C}$ NMR data were in excellent agreement with each other and with the data reported by Suzuki. ${ }^{21 a, 62}$ Additionally, the optical rotations of the synthetic materials confirm that the absolute configuration of the natural product is as represented by $(Z)-3 a$ as originally assigned by Suzuki. Additionally, the Seoul team synthesized $(E)$-notoryne $(E)$ $3 \mathrm{a}^{21 \mathrm{~b}}$ from alkene 47 . Cross-metathesis of alkene in 47 using crotonaldehyde and the Grubbs-Hoveyda catalyst 29 gave the 
corresponding $\alpha, \beta$-unsaturated aldehyde as a single $E$ isomer, ${ }^{15 \mathrm{~d}, 50}$ which on Colvin-Ohira homologation gave $(E)$-notoryne $(E)-3 \mathrm{a}$ in $88 \%$ overall yield from alkene $47^{21 \mathrm{~b}}$

Having synthesized notoryne by two independent routes, we elected to further test the computational methods for prediction/confirmation of structure of these halogenated $2,2^{\prime}$-natural products. As with the previous 2,2'-bifuranyls from Laurencia spp. we have studied, notoryne (Z)-3a contains six stereocenters, resulting in 32 diastereomeric notorynes. We decided to challenge the computational method to see if it could predict the correct structure of notoryne from the pool of 32 diastereomeric notorynes.

Upon computing the Boltzmann-weighted chemical shifts for all 32 diastereomers, we found maximum variance to stereochemical changes occurs for carbon atoms directly attached to halogen atoms and the attached protons (Figure 6). As with earlier studies, the exocyclic positions offer little for
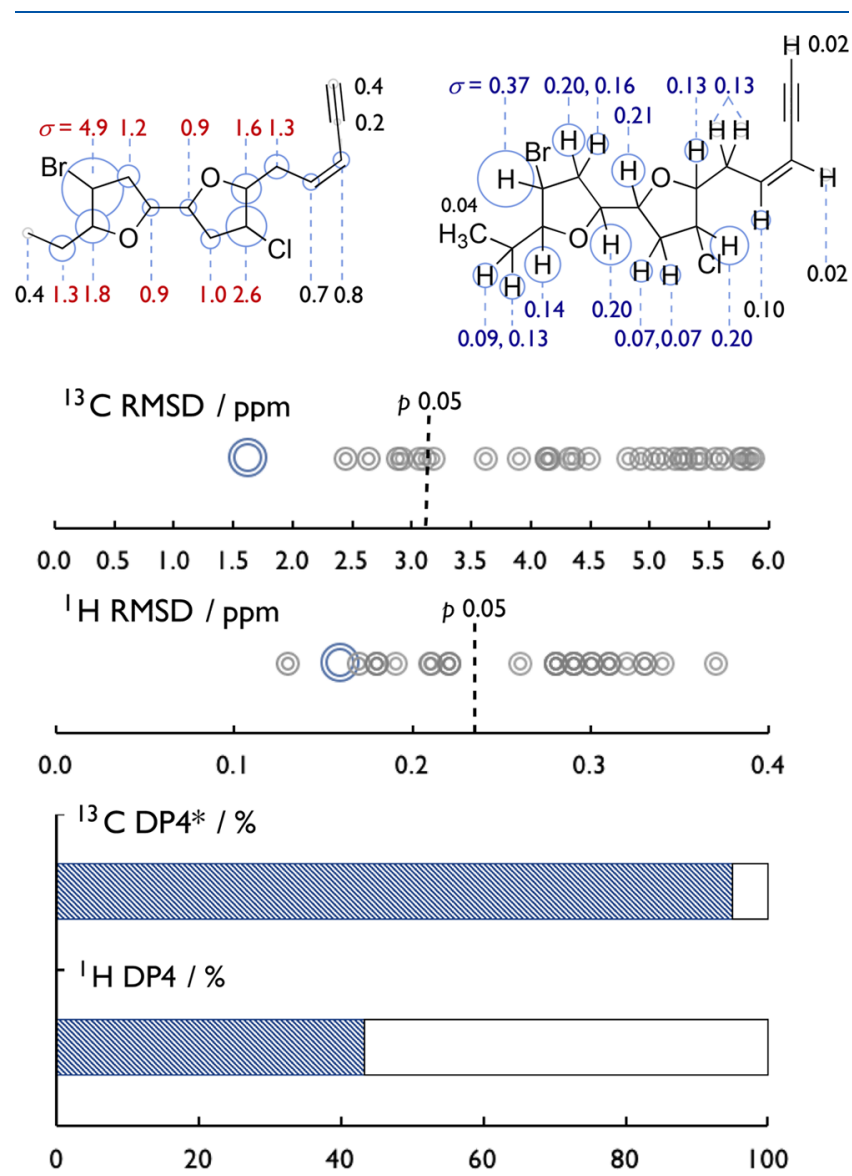

Figure 6. Comparison of computed ${ }^{13} \mathrm{C}$ and ${ }^{1} \mathrm{H}$ chemical shifts for 32 diastereomers against experimental spectra of notoryne. Data for $(Z)$ 3a are shown as blue circles in rmsd and blue area in DP4*.

predictive power and were excluded from further analysis. The stereostructure for notoryne, $(Z)-3 \mathbf{a}$, is clearly favored in the analysis of ${ }^{13} \mathrm{C}$ predictions, whereas from ${ }^{1} \mathrm{H}$ chemical shifts, it ranks in the top two structures. Again, the cumulative ${ }^{1} \mathrm{H} /{ }^{13} \mathrm{C}$ DP4 metric gives ( $Z$ )-3a as the single most likely stereostructure. The total synthesis and structure confirmation of notoryne ( $Z$ )-3a further demonstrates the utility of computational methods to not only predict but also confirm the structures of stereochemically rich, functionalized, and flexible organic molecules and natural products.

\section{CONCLUSIONS}

We have demonstrated that computational methods are able to predict the structure of a highly flexible chloroenyne natural product from $L$. majuscula containing six stereocenters from the 32 possible diastereomeric structures of the natural product. Moreover, we have synthesized three further "biomimetic" diastereomers of the natural product. Using the NMR data of these diastereomers, we have shown that the same computational methods can identify each diastereomer out of the set of 32 possible diastereomers. Key to these computational methods was to use the computed NMR chemical shift data only for those atoms that are good reporters of stereochemical information across all 32 diastereomers, that is, those atoms that show a large standard deviation in computed NMR chemical shift among the diastereomers. Furthermore, we applied both computational methods and synthesis to confirm the structure of notoryne, a further halogenated 2,2'-bifuranyl natural product isolated from Laurencia spp.

\section{EXPERIMENTAL SECTION}

General Procedures. Proton $\left({ }^{1} \mathrm{H}\right)$, carbon $\left({ }^{13} \mathrm{C}\right)$, and fluorine $\left({ }^{19} \mathrm{~F}\right)$ NMR spectra were recorded on a Bruker AV $500(500 / 125$ $\mathrm{MHz})$, Bruker AV $400(400 / 100 \mathrm{MHz})$, or Bruker DPX $300(300 / 75$ $\mathrm{MHz})$ spectrometer. Proton and carbon chemical shifts $(\delta)$ are quoted in parts per million and referenced to tetramethylsilane with residual protonated solvent as internal standard. Resonances are described as s (singlet), d (doublet), $\mathrm{t}$ (triplet), $\mathrm{q}$ (quartet), $\mathrm{m}$ (multiplet), br (broad), dd (double doublet), and so on. Coupling constants $(J)$ are given in hertz and are rounded to the nearest $0.1 \mathrm{~Hz}$. $\mathrm{H}$ and $\mathrm{H}^{\prime}$ refer to diastereotopic protons attached to the same carbon and imply no particular stereochemistry. All assignments are confimed by ${ }^{1} \mathrm{H}-{ }^{1} \mathrm{H}$ COSY and ${ }^{1} \mathrm{H}-{ }^{13} \mathrm{C}$ HSQC experiments. Low-resolution mass spectra were recorded on a Fisons Platform spectrometer (ES). High-resolution mass spectra were recorded by the mass spectrometry staff at the Chemistry Research Laboratory, University of Oxford, using a Bruker Daltronics microTOF spectrometer (ES) or a Micromass GCT (FI). The $m / z$ values are reported in Daltons with their percentage abundances and, where known, the relevant fragment ions in parentheses. High-resolution values are calculated to four decimal places from the molecular formula, with all found values being within a tolerance of $5 \mathrm{ppm}$. Infrared spectra were recorded on a Bruker Tensor 27 Fourier transform spectrometer, as a thin film on diamond ATR. Absorption maxima $\left(\nu_{\max }\right)$ are quoted in wavenumbers $\left(\mathrm{cm}^{-1}\right)$. Optical rotations were measured using a PerkinElmer 241 polarimeter in a cell of $1 \mathrm{dm}$ path length (l). TLC was performed on Merck DC-Alufolien 60F254 $0.2 \mathrm{~mm}$ precoated plates and visualized using an acidic vanillin or basic potassium permanganate dip. Retention factors $\left(R_{f}\right)$ are reported with the solvent system used in parentheses. Flash column chromatography was performed on Merck 60 silica (particle size $40-63 \mu \mathrm{m}$, pore diameter $60 \AA$ ), and the solvent system used is recorded in parentheses.

All nonaqueous reactions were carried out in oven-dried glassware under an inert atmosphere of nitrogen and employing standard techniques for handling air-sensitive materials. Solvents and commercially available reagents were dried and purified before use, as appropriate. In particular, DCM and THF were distilled from $\mathrm{CaH}_{2}$ and stored over $3 \AA$ molecular sieves. "Petrol" refers to the fraction of light petroleum ether boiling in the range of $40-60{ }^{\circ} \mathrm{C}$ unless otherwise stated. All water used experimentally was distilled, and the term "brine" refers to a saturated solution of sodium chloride in water.

(R)-3-(Benzyloxy)-3-((S)-oxiran-2-yl)propan-1-ol (18). Alkene 17 $(3 \mathrm{~g}, 14.7 \mathrm{mmol})$ was dissolved in $\mathrm{DCM} / \mathrm{MeOH}(1: 1,200 \mathrm{~mL})$ and the stirred solution cooled to $-78{ }^{\circ} \mathrm{C}$. $\mathrm{O}_{2}$ was sparged through the solution for $5 \mathrm{~min}$ followed by $\mathrm{O}_{3} / \mathrm{O}_{2}$ until a faint blue hue appeared. Then the reaction was sparged with $\mathrm{O}_{2}$ for $5 \mathrm{~min}$ and $\mathrm{PPh}_{3}$ was added $(11.6 \mathrm{~g}, 44 \mathrm{mmol})$ and the reaction stirred at $-78^{\circ} \mathrm{C}$ for $30 \mathrm{~min}$. To 
the reaction mix was added $\mathrm{NaBH}_{4}(1.6 \mathrm{~g}, 44 \mathrm{mmol})$, and the reaction was allowed to warm to rt over $2 \mathrm{~h}$. The reaction was quenched with $\mathrm{H}_{2} \mathrm{O}(100 \mathrm{~mL})$ and then diluted with DCM $(100 \mathrm{~mL})$. The aqueous phase was separated and extracted with DCM $(3 \times 50 \mathrm{~mL})$. The combined organic phases were dried $\left(\mathrm{MgSO}_{4}\right)$, filtered, and concentrated in vacuo. The crude mixture was dry-loaded onto silica and purified by rapid flash column chromatography $(2: 1 \rightarrow 1: 1$ petrol bp $30-40{ }^{\circ} \mathrm{C} /$ diethyl ether $1 \% \mathrm{NEt}_{3}$ ) to give the title compound as a colorless oil (2.81 g, $13.5 \mathrm{mmol}, 92 \%): R_{f} 0.40$ (1:1 petrol/diethyl ether); $\nu_{\max } / \mathrm{cm}^{-1}$ (thin film) $3434 \mathrm{~s} \mathrm{br}, 2875 \mathrm{~m} ;{ }^{1} \mathrm{H}$ NMR $(400 \mathrm{MHz}$ $\left.\mathrm{C}_{6} \mathrm{D}_{6}\right) \delta 7.06-7.23(\mathrm{~m}, 5 \mathrm{H}, \operatorname{Ar} H), 4.46(\mathrm{~d}, J=11.6 \mathrm{~Hz}, 1 \mathrm{H}$, $\mathrm{CHH}^{\prime} \mathrm{Ar}$ ), 4.23 (d, $\left.J=11.6 \mathrm{~Hz}, 1 \mathrm{H}, \mathrm{CHH}^{\prime} \mathrm{Ar}\right), 3.48-3.73(\mathrm{~m}, 2 \mathrm{H}$, $\mathrm{CH}_{2} \mathrm{OH}$ ), $3.24(\mathrm{dt}, J=6.8,5.6 \mathrm{~Hz}, 1 \mathrm{H}, \mathrm{CHOBn}$ ), 2.61 (ddd, $J=5.6$, $\left.3.8,2.8 \mathrm{~Hz}, 1 \mathrm{H}, \mathrm{CHOCH}_{2}\right), 2.35(\mathrm{dd}, J=5.3,2.6 \mathrm{~Hz}, 1 \mathrm{H}$, $\left.\mathrm{CHOCHH}^{\prime}\right), 2.29\left(\mathrm{dd}, J=5.4,3.8 \mathrm{~Hz}, 1 \mathrm{H}, \mathrm{CHOCHH}^{\prime}\right), 1.75-1.60$ $\left(\mathrm{m}, 3 \mathrm{H}, \mathrm{COH}, \mathrm{CH}_{2} \mathrm{CH}_{2} \mathrm{OH}\right) ;{ }^{13} \mathrm{C}\left\{{ }^{1} \mathrm{H}\right\}$ NMR $\left(100 \mathrm{MHz}, \mathrm{C}_{6} \mathrm{D}_{6}\right) \delta$ 139.0 (Ar), 128.2 (Ar), 128.0 (Ar), 127.8 (Ar), 76.9 (CHOBn), 72.4 $\left(\mathrm{CH}_{2} \mathrm{Ar}\right), 59.5\left(\mathrm{CH}_{2} \mathrm{OH}\right), 53.1\left(\mathrm{CHOCH}_{2}\right) 45.3\left(\mathrm{CHOCH}_{2}\right), 35.6$ $\left(\mathrm{CH}_{2} \mathrm{CH}_{2} \mathrm{OH}\right)$; HRMS (ESI-TOF) $m / z[\mathrm{M}+\mathrm{Na}]^{+}$calcd for $\mathrm{C}_{12} \mathrm{H}_{16} \mathrm{O}_{3} \mathrm{Na}$ 231.0992; found 231.0992; $[\alpha]_{\mathrm{D}}^{25}+32.0(c=1.0$ in $\left.\mathrm{CHCl}_{3}\right)$.

5-(((R)-3-(Benzyloxy)-3-((S)-oxiran-2-yl)propyl)thio)-1-phenyl1 H-tetrazole. $\mathrm{PPh}_{3}(4.23 \mathrm{~g}, 16.1 \mathrm{mmol})$ was dissolved in dry THF $(50 \mathrm{~mL})$ and cooled to $0{ }^{\circ} \mathrm{C}$, and DIAD $(3.2 \mathrm{~mL}, 16.1 \mathrm{mmol})$ was added dropwise to the solution and stirred at $0{ }^{\circ} \mathrm{C}$ for $15 \mathrm{~min}$. Alcohol $18(2.8 \mathrm{~g}, 13.4 \mathrm{mmol})$ was dissolved in dry THF $(25 \mathrm{~mL})$ and added dropwise to the reaction mixture followed by a wash with dry THF ( 5 $\mathrm{mL})$, and the reaction was stirred at $0{ }^{\circ} \mathrm{C}$ for $15 \mathrm{~min}$. 1-Phenyl- $1 \mathrm{H}$ tetrazole-5-thiol $(3.12 \mathrm{~g}, 17.5 \mathrm{mmol})$ was added in one portion, and the reaction mixture was warmed to rt and stirred for $16 \mathrm{~h}$. The reaction mixture was concentrated in vacuo and dry-loaded on silica. Purification by flash column chromatography (5:1 petrol/acetone) yielded the title compound as a colorless oil $(4.37 \mathrm{~g}, 11.8 \mathrm{mmol}$, $88 \%): R_{f} 0.5$ (5:1 petrol/acetone); $\nu_{\max } / \mathrm{cm}^{-1}$ (thin film) $2870 ;{ }^{1} \mathrm{H}$ NMR (400 MHz $\left.\mathrm{CDCl}_{3}\right) \delta 7.54-7.58(\mathrm{~m}, 5 \mathrm{H}, \mathrm{ArH}), 7.27-7.35(\mathrm{~m}$, $5 \mathrm{H}, \mathrm{ArH}), 4.70$ (d, $\left.J=11.4 \mathrm{~Hz}, 1 \mathrm{H}, \mathrm{CHH}^{\prime} \mathrm{Ar}\right), 4.50$ (d, $J=11.4 \mathrm{~Hz}$, $1 \mathrm{H}, \mathrm{CHH}^{\prime} \mathrm{Ar}$ ), 3.56 (ddd, $J=13.3,7.9,5.4 \mathrm{~Hz}, 1 \mathrm{H}, \mathrm{CHOBn}$ ), 3.43$3.51\left(\mathrm{~m}, 2 \mathrm{H}, \mathrm{CH}_{2} \mathrm{SAr}\right.$ ), 2.98 (ddd, $J=5.2, \mathrm{CH}_{2} \mathrm{CH}_{2} \mathrm{OH} 4.0,2.8 \mathrm{~Hz}$, $\left.1 \mathrm{H}, \mathrm{CHOCH}_{2}\right), 2.81\left(\mathrm{dd}, J=5.0,4.0 \mathrm{~Hz}, 1 \mathrm{H}, \mathrm{CHOCHH}^{\prime}\right), 2.75(\mathrm{dd}$, $\left.J=5.0,2.8 \mathrm{~Hz}, 1 \mathrm{H}, \mathrm{CHOCHH}^{\prime}\right), 2.25(\mathrm{dtd}, J=14.4,7.6,3.8 \mathrm{~Hz}, 1 \mathrm{H}$, $\mathrm{CHH}^{\prime} \mathrm{CH}_{2} \mathrm{SAr}$ ), 2.13 (dddd, $J=14.4,8.8,7.6,3.3 \mathrm{~Hz}, 1 \mathrm{H}$, $\left.\mathrm{CHH}^{\prime} \mathrm{CH}_{2} \mathrm{SAr}\right) ;{ }^{13} \mathrm{C}\left\{{ }^{1} \mathrm{H}\right\}$ NMR $\left(100 \mathrm{MHz}, \mathrm{CDCl}_{3}\right) \delta 154.2(\mathrm{Ar})$, $138.0(\mathrm{Ar}) 133.7(\mathrm{Ar}), 130.1(\mathrm{Ar}), 128.5(\mathrm{Ar}), 127.9(\mathrm{Ar}), 127.6(\mathrm{Ar})$ $123.8(\mathrm{Ar}), 76.1(\mathrm{CHOBn}), 72.5\left(\mathrm{CH}_{2} \mathrm{Ar}\right), 53.0\left(\mathrm{CHOCH}_{2}\right), 45.6$ $\left(\mathrm{CHOCH}_{2}\right), 32.12\left(\mathrm{CH}_{2} \mathrm{CH}_{2} \mathrm{SAr}\right), 29.3\left(\mathrm{CH}_{2} \mathrm{SAr}\right)$; MS (ESI-TOF) $\mathrm{m} / z 391[\mathrm{M}+\mathrm{Na}]^{+}$; HRMS (ESI-TOF) $\mathrm{m} / z[\mathrm{M}+\mathrm{Na}]^{+}$calcd for $\mathrm{C}_{19} \mathrm{H}_{20} \mathrm{~N}_{4} \mathrm{O}_{2} \mathrm{SNa}$ 391.1199; found 391.1201; $[\alpha]_{\mathrm{D}}^{25}+26.0(c=1.0$ in $\left.\mathrm{CHCl}_{3}\right)$.

5-(((R)-3-(Benzyloxy)-3-((S)-oxiran-2-yl)propyl)sulfonyl)-1-phenyl-1H-tetrazole (14). 5- $(((R)-3$-(Benzyloxy)-3- $((S)$-oxiran-2-yl)propyl)thio)-1-phenyl-1H-tetrazole $(4.3 \mathrm{~g}, 11.8 \mathrm{mmol})$ was dissolved in DCM $(200 \mathrm{~mL})$, and to the stirring solution was added $m$ CPBA $(7.2 \mathrm{~g}, 41.6 \mathrm{mmol})$, and the mixture was stirred at $\mathrm{rt}$ for 3 days. The reaction mixture was quenched with saturated aqueous $\mathrm{Na}_{2} \mathrm{~S}_{2} \mathrm{O}_{3}$ (1 $\mathrm{mL})$ and then with saturated aqueous $\mathrm{NaHCO}_{3}(200 \mathrm{~mL})$. The aqueous layer was separated and extracted with DCM $(3 \times 100 \mathrm{~mL})$. The combined organic layers were dried $\left(\mathrm{Na}_{2} \mathrm{SO}_{4}\right)$, filtered, and concentrated in vacuo. Purification via flash column chromatography (DCM) gave the title compound as white needles $(2.85 \mathrm{~g}, 7.1 \mathrm{mmol}$, 60\%): $R_{f} 0.52$ (5:1 petrol/acetone); $\mathrm{mp} 90-92{ }^{\circ} \mathrm{C} ; \nu_{\max } / \mathrm{cm}^{-1}$ (thin film) 2918s, 1342s, 1150s; ${ }^{1} \mathrm{H}$ NMR (400 $\left.\mathrm{MHz} \mathrm{CDCl}_{3}\right) \delta 7.56-7.70$ (m, 5H, ArH), 7.29-7.38 (m, 5H, ArH), 4.69 (d, $J=11.6 \mathrm{~Hz}, 1 \mathrm{H}$, $\mathrm{CHH}^{\prime} \mathrm{Ar}$ ), 4.52 (d, $J=11.6 \mathrm{~Hz}, 1 \mathrm{H}, \mathrm{CHH}^{\prime} \mathrm{Ar}$ ), 3.93 (ddd, $J=14.9$, $10.2,5.3 \mathrm{~Hz}, 1 \mathrm{H}, \mathrm{CH} H^{\prime} \mathrm{SAr}$ ), 3.81 (ddd, $J=14.9,10.3,5.6 \mathrm{~Hz}, 1 \mathrm{H}$, $\mathrm{CHH}^{\prime} \mathrm{SAr}$ ), 3.47 (ddd, $J=8.3,5.6,4.3 \mathrm{~Hz}, 1 \mathrm{H}, \mathrm{CHOBn}$ ), 2.96 (ddd, $J$ $\left.=5.6,3.8,2.5 \mathrm{~Hz}, 1 \mathrm{H}, \mathrm{CHOCH}_{2}\right), 2.82(\mathrm{dd}, J=5.0,3.8 \mathrm{~Hz}, 1 \mathrm{H}$, $\left.\mathrm{CHOCHH}^{\prime}\right), 2.72\left(\mathrm{dd}, J=5.0,2.5 \mathrm{~Hz}, 1 \mathrm{H}, \mathrm{CHOCH} H^{\prime}\right), 2.37$ (dddd, $J=14.4,9.9,5.6,4.3 \mathrm{~Hz}, 1 \mathrm{H}, \mathrm{CHH}^{\prime} \mathrm{CH}_{2} \mathrm{SAr}$ ) 2.24 (dddd, $J=14.4$, 10.3, 8.3, $\left.5.3 \mathrm{~Hz}, 1 \mathrm{H}, \mathrm{CHH} \mathrm{H}^{\prime} \mathrm{CH}_{2} \mathrm{SAr}\right) ;{ }^{13} \mathrm{C}\left\{{ }^{1} \mathrm{H}\right\}$ NMR (100 MHz, $\left.\mathrm{CDCl}_{3}\right) \delta 153.3(\mathrm{Ar}) 137.5(\mathrm{Ar}), 133.0(\mathrm{Ar}), 131.5(\mathrm{Ar}), 129.7$ (Ar),
128.6 (Ar), 128.1 (Ar), 127.9 (Ar), 125.1 (Ar), $75.6\left(\mathrm{CH}_{2} \mathrm{Ar}\right), 72.4$ (CHOBn), $52.6\left(\mathrm{CH}_{2} \mathrm{SAr}\right), 52.3\left(\mathrm{CHOCH}_{2}\right), 45.7\left(\mathrm{CHOCH}_{2}\right), 25.5$ $\left(\mathrm{CH}_{2} \mathrm{CH}_{2} \mathrm{SAr}\right.$ ); MS (ESI-TOF) $m / z 423[\mathrm{M}+\mathrm{Na}]^{+}$; HRMS (ESITOF) $m / z[\mathrm{M}+\mathrm{Na}]^{+}$calcd for $\mathrm{C}_{19} \mathrm{H}_{20} \mathrm{~N}_{4} \mathrm{O}_{4} \mathrm{SNa}$ 423.1097; found 423.1089; $[\alpha]_{\mathrm{D}}^{25}+14.5\left(c=1.0\right.$ in $\left.\mathrm{CHCl}_{3}\right)$.

tert-Butyl-(((3R,4S)-4-((4-methoxybenzyl)oxy)hept-6-en-3-yl)oxy)dimethylsilane (20). The known alcohol $(3 R, 4 S)-4-(4-$ methoxybenzyloxy)hept-6-en-3-ol was readily prepared from the epoxy alcohol (+)-16 via the p-methoxybenzyl ether 19 according to our previously reported route. ${ }^{15 \mathrm{~d}}(3 R, 4 S)-4$-(4-Methoxybenzyloxy)hept-6-en-3-ol thus prepared $(3.3 \mathrm{~g}, 13.2 \mathrm{mmol})$ was dissolved in dry DMF $(100 \mathrm{~mL})$, then imidazole $(1.97 \mathrm{~g}, 29.6 \mathrm{mmol})$ and TBSCl $(2.98 \mathrm{~g}, 19.8 \mathrm{mmol})$ were added. The reaction mixture was heated to $60{ }^{\circ} \mathrm{C}$ for $16 \mathrm{~h}$. The reaction was cooled to $\mathrm{rt}$ and then quenched with water $(50 \mathrm{~mL})$. The aqueous layer was separated and extracted with diethyl ether $(3 \times 75 \mathrm{~mL})$. The combined organic layers were washed with water $(3 \times 50 \mathrm{~mL})$ and brine $(50 \mathrm{~mL})$, dried $\left(\mathrm{MgSO}_{4}\right)$, filtered, and concentrated in vacuo. Purification by flash column chromatography (20:1 petrol bp $30-40{ }^{\circ} \mathrm{C} /$ diethyl ether) gave the title compound as a colorless oil $(4.81 \mathrm{~g}, 13.0 \mathrm{mmol}, 99 \%): R_{f} 0.62(20: 1$ petrol/diethyl ether); $\nu_{\max } / \mathrm{cm}^{-1}$ (thin film) $3076 \mathrm{~m}, 2957 \mathrm{~s}, 2931 \mathrm{~s}$, $2857 \mathrm{~s}, 1641 \mathrm{~m} ;{ }^{1} \mathrm{H}$ NMR $\left(400 \mathrm{MHz} \mathrm{CDCl}_{3}\right) \delta 7.28(\mathrm{~d}, J=8.6 \mathrm{~Hz}$, $2 \mathrm{H}, \operatorname{ArH}), 6.87(\mathrm{~d}, J=8.6 \mathrm{~Hz}, 2 \mathrm{H}, \operatorname{Ar} H), 5.90(\mathrm{ddt}, J=17.0,10.0,7.0$ $\left.\mathrm{Hz}, 1 \mathrm{H}, \mathrm{CH}=\mathrm{CH}_{2}\right), 5.10(\mathrm{dq}, J=17.0,1.7 \mathrm{~Hz}, 1 \mathrm{H}, \mathrm{CH}=\mathrm{CHH})$, $5.05(\mathrm{ddt}, J=10.0,2.3,1.1 \mathrm{~Hz}, 1 \mathrm{H}, \mathrm{CH}=\mathrm{CHH}) 4.59(\mathrm{~d}, J=11.3 \mathrm{~Hz}$, $\left.1 \mathrm{H}, \mathrm{CHH}^{\prime} \mathrm{Ar}\right), 4.49\left(\mathrm{~d}, J=11.3 \mathrm{~Hz}, 1 \mathrm{H}, \mathrm{CHH}^{\prime} \mathrm{Ar}\right), 3.81(\mathrm{~s}, 3 \mathrm{H}$, $\mathrm{OMe}$ ), 3.69 (dt, $J=6.6,4.3 \mathrm{~Hz}, 1 \mathrm{H}, \mathrm{CHOTBS}), 3.41(\mathrm{td}, J=6.0,4.3$ $\mathrm{Hz}, 1 \mathrm{H}, \mathrm{CHOPMB}), 2.33\left(\mathrm{dd} J=7.0,6.0 \mathrm{~Hz}, 2 \mathrm{H}, \mathrm{CH}_{2} \mathrm{CH}=\mathrm{CH}_{2}\right)$, $1.43-1.71\left(\mathrm{~m}, 2 \mathrm{H}, \mathrm{CH}_{3} \mathrm{CH}_{2}\right), 0.92\left(\mathrm{~s}, 9 \mathrm{H}, \mathrm{CMe} e_{3}\right), 0.90(\mathrm{t}, J=7.5 \mathrm{~Hz}$, $\left.3 \mathrm{H}, \mathrm{CH}_{3} \mathrm{CH}_{2}\right), 0.07\left(\mathrm{~s}, 3 \mathrm{H}, \mathrm{SiCH}_{3}\right), 0.06\left(\mathrm{~s}, 3 \mathrm{H}, \mathrm{SiCH}_{3}\right) ;{ }^{13} \mathrm{C}\left\{{ }^{1} \mathrm{H}\right\}$ NMR $\left(100 \mathrm{MHz} \mathrm{CDCl}_{3}\right) \delta 159.0(\mathrm{Ar}), 136.0\left(\mathrm{CH}=\mathrm{CH}_{2}\right), 131.1$ (Ar), $129.3(\mathrm{Ar}), 116.4\left(\mathrm{CH}=\mathrm{CH}_{2}\right), 113.6(\mathrm{Ar}), 81.4(\mathrm{CHOPMB})$, 74.9 (CHOTBS), $72.0\left(\mathrm{CH}_{2} \mathrm{Ar}\right), 55.3(\mathrm{OMe}), 35.6\left(\mathrm{CH}_{2} \mathrm{CH}=\mathrm{CH}_{2}\right)$, 26.0, $\left(\mathrm{CMe}_{3}\right), 25.5\left(\mathrm{CH}_{3} \mathrm{CH}_{2}\right), 18.2(\mathrm{SiC}), 9.7\left(\left(\mathrm{CH}_{3}\right)_{3} \mathrm{C}\right),-4.3$ $\left(\mathrm{SiCH}_{3}\right),-4.5\left(\mathrm{SiCH}_{3}\right)$; MS (ESI-TOF) $\mathrm{m} / z 387[\mathrm{M}+\mathrm{Na}]^{+}$; HRMS (ESI-TOF) $m / z[\mathrm{M}+\mathrm{Na}]^{+}$calcd for $\mathrm{C}_{21} \mathrm{H}_{36} \mathrm{O}_{3} \mathrm{SiNa}$ 387.2326; found 387.2326; $[\alpha]_{\mathrm{D}}^{25}-11.9\left(c=1.0\right.$ in $\left.\mathrm{CHCl}_{3}\right)$.

(3S,4R)-4-((tert-Butyldimethylsilyl)oxy)-3-((4-methoxybenzyl)oxy)hexanal (15). Alkene $20(3.68 \mathrm{~g}, 10 \mathrm{mmol})$ was dissolved in DCM $(300 \mathrm{~mL})$, cooled to $-78{ }^{\circ} \mathrm{C}$, and sparged with $\mathrm{O}_{2}$ for $2 \mathrm{~min}$. $\mathrm{O}_{3} / \mathrm{O}_{2}$ was then bubbled through the stirred solution until a faint blue color appeared, and excess ozone was then sparged out with $\mathrm{O}_{2}$ for 5 min before adding $\mathrm{PPh}_{3}(7.9 \mathrm{~g}, 30 \mathrm{mmol})$. The reaction mixture was allowed to warm to rt over $16 \mathrm{~h}$ before being concentrated in vacuo and dry-loaded onto silica. Purification via rapid column chromatography $(16: 1 \rightarrow 8: 1 \mathrm{petrol} /$ diethyl ether) yielded the title compound as a colorless oil $(3.60 \mathrm{~g}, 9.8 \mathrm{mmol}, 98 \%): R_{f} 0.63$ (10:1 petrol/ethyl acetate); $\nu_{\mathrm{max}} / \mathrm{cm}^{-1}$ (thin film) 2931s, 2858s, $1725 \mathrm{~s}$; ${ }^{1} \mathrm{H}$ NMR (400 $\left.\mathrm{MHz} \mathrm{CDCl}_{3}\right) \delta 9.80(\mathrm{t}, J=2.0 \mathrm{~Hz}, 1 \mathrm{H}, \mathrm{CHO}), 7.24-7.30(\mathrm{~m}$, $2 \mathrm{H}, \mathrm{ArH}), 6.86-6.92(\mathrm{~m}, 2 \mathrm{H}, \mathrm{ArH}), 4.57(\mathrm{~d}, J=11.2 \mathrm{~Hz}, 1 \mathrm{H}$, $\left.\mathrm{CHH}^{\prime} \mathrm{Ar}\right), 4.45\left(\mathrm{~d}, J=11.2 \mathrm{~Hz}, 1 \mathrm{H}, \mathrm{CH} H^{\prime} \mathrm{Ar}\right), 3.87(\mathrm{dt}, J=7.4,3.6$ $\mathrm{Hz}, 1 \mathrm{H}, \mathrm{CHOPMB}), 3.80(\mathrm{~s}, 3 \mathrm{H}, \mathrm{OMe}), 3.78(\mathrm{dt}, J=7.0,3.6 \mathrm{~Hz}, 1 \mathrm{H}$, CHOTBS), 2.69 (ddd, $J=16.7,7.0,2.0 \mathrm{~Hz}, 1 \mathrm{H}, \mathrm{CHH}^{\prime} \mathrm{CHO}$ ), 2.56 (ddd, $\left.J=16.7,7.0,2.0 \mathrm{~Hz}, 1 \mathrm{H}, \mathrm{CH} H^{\prime} \mathrm{CHO}\right), 1.37-1.62(\mathrm{~m}, 2 \mathrm{H}$, $\left.\mathrm{CH}_{3} \mathrm{CH}_{2}\right), 0.91\left(\mathrm{t}, J=7.3 \mathrm{~Hz}, 3 \mathrm{H}, \mathrm{CH}_{3} \mathrm{CH}_{2}\right), 0.90\left(\mathrm{~s}, 9 \mathrm{H}, \mathrm{CMe}_{3}\right)$, $0.08\left(\mathrm{~s}, 3 \mathrm{H}, \mathrm{SiCH}_{3}\right), 0.07\left(\mathrm{~s}, 3 \mathrm{H}, \mathrm{SiCH}_{3}\right) ;{ }^{13} \mathrm{C}\left\{{ }^{1} \mathrm{H}\right\} \mathrm{NMR}(100 \mathrm{MHz}$, $\left.\mathrm{CDCl}_{3}\right) \delta 202.0(\mathrm{C}=\mathrm{O}), 159.2(\mathrm{Ar}), 130.2(\mathrm{Ar}), 129.4(\mathrm{Ar}), 113.8$ (Ar), 76.8 (CHOPMB), 74.5 (CHOTBS), $71.6\left(\mathrm{CH}_{2} \mathrm{Ar}\right) 55.3(\mathrm{OMe})$,

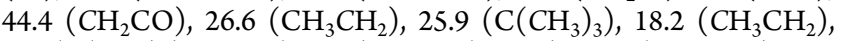

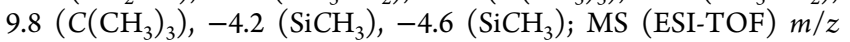
$389[\mathrm{M}+\mathrm{Na}]^{+}$; HRMS (ESI-TOF) $\mathrm{m} / z[\mathrm{M}+\mathrm{N}]^{+}$calcd for $\mathrm{C}_{20} \mathrm{H}_{34} \mathrm{O}_{4} \mathrm{SiNa} 389.2119$; found 389.2117; $[\alpha]_{\mathrm{D}}^{25}-15.6(c=1.0$ in $\left.\mathrm{CHCl}_{3}\right)$.

(((3R,4S,9R,E)-9-(Benzyloxy)-4-((4-methoxybenzyl)oxy)-9-((S)-oxiran-2-yl)non-6-en-3-yl)oxy)(tert-butyl)dimethylsilane (13). To a stirred solution of sulfone $14(1.95 \mathrm{~g}, 4.88 \mathrm{mmol})$, in dry DME (60 $\mathrm{mL})$ cooled to $-78^{\circ} \mathrm{C}$, was added NaHDMS $(3.42 \mathrm{~mL}, 2 \mathrm{M}$ in THF, $6.83 \mathrm{mmol}$ ) dropwise. The reaction was stirred for $15 \mathrm{~min}$, and a solution of aldehyde $15(3.57 \mathrm{~g}, 9.75 \mathrm{mmol})$ in DME $(20 \mathrm{~mL})$ was added dropwise over $15 \mathrm{~min}$ followed by a wash of DME $(10 \mathrm{~mL})$. 
The reaction mixture was stirred at $-78^{\circ} \mathrm{C}$ for $1 \mathrm{~h}$. The reaction was then warmed to $\mathrm{rt}$ and stirred for $2 \mathrm{~h}$. The reaction mixture was quenched with saturated aqueous $\mathrm{NH}_{4} \mathrm{Cl}(30 \mathrm{~mL})$ and then diluted with $\mathrm{H}_{2} \mathrm{O}(20 \mathrm{~mL})$ and EtOAc $(50 \mathrm{~mL})$. The aqueous layer was separated and extracted with EtOAc $(3 \times 50 \mathrm{~mL})$. The combined organic layers were dried $\left(\mathrm{MgSO}_{4}\right)$, filtered, and concentrated in vacuo. Purification by flash column chromatography $(20: 1 \rightarrow 15: 1 \rightarrow$ 10:1 petrol/ethyl acetate) gave the title compound as a colorless oil $(2.1 \mathrm{~g}, 3.9 \mathrm{mmol}, 80 \%)$ and recovered aldehyde $(1.2 \mathrm{~g}, 3.2 \mathrm{mmol}): R_{f}$ 0.49 (10:1 petrol/ethyl acetate); $\nu_{\max } / \mathrm{cm}^{-1}$ (thin film) 2930s, 2856 m, 1613w; ${ }^{1} \mathrm{H}$ NMR (400 MHz CDCl $\left.{ }_{3}\right) \delta 7.24-7.36(\mathrm{~m}, 7 \mathrm{H}, \mathrm{ArH})$, 6.84-6.88 (m, 2H, $\mathrm{ArH}), 5.54-5.67(\mathrm{~m}, 2 \mathrm{H}, \mathrm{CH}=\mathrm{CH}), 4.63(\mathrm{~d}, J=$ $\left.11.8 \mathrm{~Hz}, 1 \mathrm{H}, \mathrm{CHH}^{\prime} \mathrm{Ar}\right), 4.58,\left(\mathrm{~d}, J=11.2 \mathrm{~Hz}, 1 \mathrm{H}, \mathrm{CHH} \mathrm{H}^{\prime} \mathrm{Ar}\right), 4.53$ (d, $\left.J=11.8 \mathrm{~Hz}, 1 \mathrm{H}, \mathrm{CHH}^{\prime} \mathrm{Ar}\right), 4.46\left(\mathrm{~d}, J=11.2 \mathrm{~Hz}, 1 \mathrm{H}, \mathrm{CH} H^{\prime} \mathrm{Ar}\right), 3.80$ (s, 3H, OMe), $3.68(\mathrm{dt}, J=6.5,4.3 \mathrm{~Hz}, 1 \mathrm{H}, \mathrm{CHOTBS}), 3.37$ (td, $J=$ $5.9,4.3 \mathrm{~Hz}, 1 \mathrm{H}, \mathrm{CHOPMB}), 3.31(\mathrm{dt}, J=6.7,5.2 \mathrm{~Hz}, 1 \mathrm{H}, \mathrm{CHOBn})$, 2.97 (ddd, $J=5.2,4.0,2.8 \mathrm{~Hz}, 1 \mathrm{H}, \mathrm{CHOCH}_{2}$ ), 2.77 (dd, $J=5.3,4.0$ $\left.\mathrm{Hz}, 1 \mathrm{H}, \mathrm{CHOCHH}^{\prime}\right), 2.73\left(\mathrm{dd}, J=5.3,2.8 \mathrm{~Hz}, 1 \mathrm{H}, \mathrm{CHOCHH}^{\prime}\right)$, 2.33-2.47 (m, 2H, CHOBnCH $\left.{ }_{2}\right), 2.29\left(\mathrm{~m}, 2 \mathrm{H}, \mathrm{CHOPMBCH}_{2}\right)$, 1.58-1.69 (m, $\left.1 \mathrm{H}, \mathrm{CH}_{3} \mathrm{CHH}\right), 1.45-1.55\left(\mathrm{~m}, 1 \mathrm{H}, \mathrm{CH}_{3} \mathrm{CHH}\right), 0.92$ $\left(\mathrm{s}, 9 \mathrm{H}, \mathrm{CM} e_{3}\right) 0.91\left(\mathrm{t}, J=8.4 \mathrm{~Hz}, 3 \mathrm{H}, \mathrm{CH}_{3} \mathrm{CH}_{2}\right), 0.06(\mathrm{~s}, 3 \mathrm{H}$, $\left.\mathrm{SiCH}_{3}\right), 0.06\left(\mathrm{~s}, 3 \mathrm{H}, \mathrm{SiCH}_{3}\right) ;{ }^{13} \mathrm{C}\left\{{ }^{1} \mathrm{H}\right\} \operatorname{NMR}\left(100 \mathrm{MHz}, \mathrm{CDCl}_{3}\right) \delta$ $159.0(\mathrm{Ar}), 138.5(\mathrm{Ar}), 131.1(\mathrm{Ar}), 130.4(\mathrm{CH}=\mathrm{CH}), 129.3(\mathrm{Ar})$, $128.3(\mathrm{Ar}), 127.6(\mathrm{Ar}) 127.2(\mathrm{Ar}) 127.2(\mathrm{CH}=\mathrm{CH}) 113.6(\mathrm{Ar}), 81.7$ (CHOPMB) 78.0 (CHOBn), 75.0 (CHOTBS), $72.2\left(\mathrm{CH}_{2} \mathrm{Ar}\right), 72.0$ $\left(\mathrm{CH}_{2} \mathrm{Ar}\right), 55.3(\mathrm{OMe}), 53.2\left(\mathrm{CHOCH}_{2}\right), 45.6\left(\mathrm{CHOCH}_{2}\right), 36.1$ $\left.\left(\mathrm{CHOBnCH}_{2}\right), 34.1(\mathrm{CHOPMBCH})_{2}\right), 26.0\left(\mathrm{CMe}_{3}\right) 25.5\left(\mathrm{CH}_{3} \mathrm{CH}_{2}\right)$, $18.2\left(\mathrm{CMe}_{3}\right), 9.8\left(\mathrm{CH}_{3} \mathrm{CH}_{2}\right),-4.2\left(\mathrm{SiCH}_{3}\right),-4.5\left(\mathrm{SiCH}_{3}\right) ; \mathrm{MS}$ (ESITOF) $m / z 563[\mathrm{M}+\mathrm{Na}]^{+}$; HRMS (ESI-TOF) $\mathrm{m} / z[\mathrm{M}+\mathrm{Na}]^{+}$calcd for $\mathrm{C}_{32} \mathrm{H}_{48} \mathrm{O}_{5} \mathrm{SiNa} 563.3163$; found 563.3174; $[\alpha]_{\mathrm{D}}^{20}-17.2(c=1.0$ in $\left.\mathrm{CHCl}_{3}\right)$.

(1R,3R,4R,6S,7R)-1-(Benzyloxy)-7-((tert-butyldimethylsilyl)oxy)-6((4-methoxybenzyl)oxy)-1-((S)-oxiran-2-yl)nonane-3,4-diol (12). Alkene 13 (400 mg, $0.74 \mathrm{mmol})$, methanesulfonamide $(211 \mathrm{mg}, 2.22$ $\mathrm{mmol}),\left(\mathrm{DHQD}_{2}\right)$ PHAL $(55.6 \mathrm{mg}, 0.074 \mathrm{mmol}), \mathrm{K}_{3} \mathrm{Fe}(\mathrm{CN})_{6}(730$ $\mathrm{mg}, 0.28 \mathrm{mmol})$, and $\mathrm{K}_{2} \mathrm{CO}_{3}(307 \mathrm{mg}, 2.22 \mathrm{mmol})$ were dissolved in ${ }^{t} \mathrm{BuOH} / \mathrm{H}_{2} \mathrm{O}(8 \mathrm{~mL}: 8 \mathrm{~mL}) . \mathrm{K}_{2} \mathrm{OsO}_{4}(\mathrm{OH})_{2}(2.73 \mathrm{mg}, 7.4 \mu \mathrm{mol})$ was added and the reaction stirred for $24 \mathrm{~h}$. Sodium sulfite $(279 \mathrm{mg}, 2.22$ $\mathrm{mmol}$ ) was added, and the reaction was stirred at $\mathrm{rt}$ for 30 min. $\mathrm{H}_{2} \mathrm{O}$ $(10 \mathrm{~mL})$ was added, and the aqueous layer was extracted with EtOAc $(3 \times 10 \mathrm{~mL})$. The combined organic phases were then washed with aqueous $\mathrm{NaOH}(10 \mathrm{~mL}, 0.1 \mathrm{M})$, and the aqueous layer was back extracted with EtOAc $(15 \mathrm{~mL})$. The combined organic layers were dried $\left(\mathrm{Na}_{2} \mathrm{SO}_{4}\right)$, filtered, and concentrated in vacuo. Purification by flash column chromatography (3:1 petrol/ethyl acetate) gave the title compound as a partially separable mixture of colorless oils $(377 \mathrm{mg}$ total, 0.66 mmol 91\% 6:1 3R,4R:3S,4S diastereomers from ${ }^{1} \mathrm{H}$ NMR analysis of the crude, from which $268 \mathrm{mg}, 0.47 \mathrm{mmol}, 64 \%$ could be obtained in pure form): $R_{f} 0.38$ (3:1 petrol/ethyl acetate); $\nu_{\max } / \mathrm{cm}^{-1}$ (thin film) 3471m, 2930s, 2859s; ${ }^{1} \mathrm{H}$ NMR (400 MHz C $\left.\mathrm{D}_{6}\right) \delta 7.15-$ $7.47(\mathrm{~m}, 7 \mathrm{H}, \mathrm{ArH}), 6.85-6.91(\mathrm{~m}, 2 \mathrm{H}, \operatorname{Ar} H), 4.72(\mathrm{~d}, J=11.5 \mathrm{~Hz}$, $1 \mathrm{H}, \mathrm{CH} \mathrm{H}^{\prime} \mathrm{Ar}$ ), $4.67\left(\mathrm{~d}, J=10.9 \mathrm{~Hz}, 1 \mathrm{H}, \mathrm{CHH}^{\prime} \mathrm{Ar}\right), 4.54$ (d, $J=11.5$ $\left.\mathrm{Hz}, 1 \mathrm{H}, \mathrm{CHH}^{\prime} \mathrm{Ar}\right), 4.39$ (d, $\left.J=10.9 \mathrm{~Hz}, 1 \mathrm{H}, \mathrm{CHH}^{\prime} \mathrm{Ar}\right), 4.26(1 \mathrm{H}, \mathrm{br}$, $\mathrm{OH}), 4.06(1 \mathrm{H}, \mathrm{br}, \mathrm{CHOH}), 3.82-3.87(\mathrm{~m}, 2 \mathrm{H}, \mathrm{CHOH}, \mathrm{CHOBn})$, 3.76 (ddd, $J=1.9,4.9,7.7 \mathrm{~Hz}, 1 \mathrm{H}, \mathrm{CHOTBS}), 3.62$ (ddd, $J=1.9,3.0$, $8.5 \mathrm{~Hz}, 1 \mathrm{H}, \mathrm{CHOPMB}$ ), 3.38 (s, 3H, OMe), 2.86 (ddd, $J=2.7,3.8$, $\left.6.3 \mathrm{~Hz}, 1 \mathrm{H}, \mathrm{CHOCH}_{2}\right), 2.81($ br s, $1 \mathrm{H}, \mathrm{OH}), 2.56(\mathrm{dd}, J=2.7,5.5$ $\left.\mathrm{Hz}, 1 \mathrm{H}, \mathrm{CHOCHH^{ \prime }}\right), 2.45,\left(\mathrm{dd} J=3.8,5.5 \mathrm{~Hz}, 1 \mathrm{H}, \mathrm{CHOCHH}^{\prime}\right)$, 2.02-2.16 (m, $\left.2 \mathrm{H}, 2 \times \mathrm{CHH}^{\prime} \mathrm{CHOH}\right), 1.91$ (ddd, $J=14.2,9.5,3.0$ $\mathrm{Hz}, 1 \mathrm{H}$, CHOPMBCHH' $\left.\mathrm{H}^{\prime}\right), 1.78$ (ddd, $J=15.8,3.5,2.5 \mathrm{~Hz}, 1 \mathrm{H}$, CHOBnCHH $\left.H^{\prime}\right), 1.64\left(\mathrm{dq}, J=14.0,7.6 \mathrm{~Hz}, 1 \mathrm{H}, \mathrm{CH}_{3} \mathrm{CHH}^{\prime}\right), 1.42$ (dqd, $\left.J=14.0,7.5,4.9 \mathrm{~Hz}, 1 \mathrm{H}, \mathrm{CH}_{3} \mathrm{CHH}^{\prime}\right), 1.11\left(\mathrm{~s}, 9 \mathrm{H}, \mathrm{CMe}_{3}\right), 0.97$ (t, $\left.J=7.5 \mathrm{~Hz}, 3 \mathrm{H}, \mathrm{CH}_{3} \mathrm{CH}_{2}\right), 0.25\left(\mathrm{~s}, 3 \mathrm{H}, \mathrm{SiCH}_{3}\right), 0.19\left(\mathrm{~s}, 3 \mathrm{H}, \mathrm{CH}_{3}\right)$; ${ }^{13} \mathrm{C}\left\{{ }^{1} \mathrm{H}\right\}$ NMR (100 MHz, $\left.\mathrm{C}_{6} \mathrm{D}_{6}\right) \delta 159.9(\mathrm{Ar}), 139.4(\mathrm{Ar}), 130.4$ (Ar), 130.0 (Ar), 128.5 (Ar), 127.6 (Ar), 128.5 (Ar), 114.2 (Ar), 82.3 (CHOBn), 76.0 (CHOPMB), 75.9 (CHOTBS), 73.2 (CHOH), 73.0 $\left(\mathrm{CH}_{2} \mathrm{Ar}\right), 72.0(\mathrm{CHOH}), 71.0\left(\mathrm{CH}_{2} \mathrm{Ar}\right), 54.7(\mathrm{OMe}), 53.6$ $\left(\mathrm{CHOCH}_{2}\right), 45.2\left(\mathrm{CHOCH}_{2}\right), 37.3\left(\mathrm{CHOPMBCH}_{2}\right), 33.1$ $\left(\mathrm{CHOBnCH}_{2}\right), 26.5\left(\mathrm{CH}_{3} \mathrm{CH}_{2}\right), 26.2\left(\mathrm{CMe}_{3}\right), 18.5\left(\mathrm{CMe}_{3}\right), 10.9$ $\left(\mathrm{CH}_{3} \mathrm{CH}_{2}\right),-3.9(\mathrm{SiMe}),-4.6(\mathrm{SiMe})$; HRMS (ESI-TOF) $\mathrm{m} / z[\mathrm{M}+$
$\mathrm{Na}]^{+}$calcd for $\mathrm{C}_{32} \mathrm{H}_{50} \mathrm{O}_{7} \mathrm{SiNa} 597.3218$; found 597.3221; $[\alpha]_{\mathrm{D}}^{20}-4.0$ ( $c=1.0$ in $\mathrm{CHCl}_{3}$ ).

(1R,3S,4R)-1-((2R,4R,5R)-4-(Benzyloxy)-5-(hydroxymethyl)tetrahydrofuran-2-yl)-4-((tert-butyldimethylsilyl)oxy)-3-((4-methoxybenzyl)oxy)hexan-1-ol (21). To a solution of diol $12(1.3 \mathrm{~g}, 2.3$ $\mathrm{mmol})$ in DCM $(18 \mathrm{~mL})$ at $0{ }^{\circ} \mathrm{C}$ was added a solution of camphorsulfonic acid $(10.6 \mathrm{mg}, 0.05 \mathrm{mmol})$ in DCM $(1.1 \mathrm{~mL})$. The reaction was stirred for $2 \mathrm{~h}$ at $0{ }^{\circ} \mathrm{C}$, and the cold reaction mixture was immediately purified by flash column chromatography (1:1 petrol/ ethyl acetate) to give the title compound as a colorless oil (1.12 g, 2.0 mmol, 86\%): $R_{f}$ (5:1 petrol/ethyl acetate); $\nu_{\max } / \mathrm{cm}^{-1}$ (thin film) 3419br, 2930s, 2858s; ${ }^{1} \mathrm{H}$ NMR (400 MHz $\left.\mathrm{CDCl}_{3}\right) \delta 7.24-7.39(\mathrm{~m}$, $7 \mathrm{H}, \operatorname{ArH}), 6.85-6.89(\mathrm{~m}, 2 \mathrm{H}, \operatorname{ArH}), 4.70(\mathrm{~d}, J=11.0 \mathrm{~Hz}, 1 \mathrm{H}$, $\left.\mathrm{CHH}^{\prime} \mathrm{Ar}\right), 4.62\left(\mathrm{~d}, J=11.9 \mathrm{~Hz}, 1 \mathrm{H}, \mathrm{CHH}^{\prime} \mathrm{Ar}\right), 4.42(\mathrm{~d}, J=11.9 \mathrm{~Hz}$, $\left.1 \mathrm{H}, \mathrm{CHH}^{\prime} \mathrm{Ar}\right), 4.41$ (d, $\left.J=11.0 \mathrm{~Hz}, \mathrm{CHH}^{\prime} \mathrm{Ar}\right), 4.25(\mathrm{q}, J=6.2 \mathrm{~Hz}$, $1 \mathrm{H}, \mathrm{CHOBn}), 3.95$ (dt, $J=6.1,4.5 \mathrm{~Hz}, 1 \mathrm{H}, \mathrm{CHORCHOH}), 3.81-$ $3.88\left(\mathrm{~m}, 4 \mathrm{H}, \mathrm{CHOH}, \mathrm{CHORCH}_{2} \mathrm{OH}\right), 3.80(\mathrm{~s}, 3 \mathrm{H}, \mathrm{OMe}), 3.75$ (ddd, $J=7.1,5.0,2.0 \mathrm{~Hz}, 1 \mathrm{H}, \mathrm{CHOTBS}$ ), 3.64 (ddd, $J=8.1,3.8,2.0$ $\mathrm{Hz}, 1 \mathrm{H}, \mathrm{CHOPMB}), 2.80$ (br, $1 \mathrm{H}, \mathrm{OH}), 2.14$ (ddd, $J=13.4,7.0,6.2$ $\mathrm{Hz}, 1 \mathrm{H}, \mathrm{CHH}^{\prime} \mathrm{CHOBn}$ ), 2.00 (ddd, $J=13.4,8.0,6.2 \mathrm{~Hz}, 1 \mathrm{H}$, $\left.\mathrm{CHH}^{\prime} \mathrm{CHOBn}\right), 1.80\left(\mathrm{dt}, \mathrm{J}=14.8,8.1 \mathrm{~Hz}, 1 \mathrm{H}, \mathrm{CHH}^{\prime} \mathrm{CHOPMB}\right)$, $1.70\left(\mathrm{dt}, J=14.8,3.8 \mathrm{~Hz}, 1 \mathrm{H}, \mathrm{CHH}^{\prime} \mathrm{OPMB}\right), 1.51-1.63(\mathrm{~m}, 1 \mathrm{H}$, $\left.\mathrm{CH}_{3} \mathrm{CHH}^{\prime}\right), 1.40-1.50\left(\mathrm{~m}, \mathrm{CH}_{3} \mathrm{CHH}^{\prime}\right), 0.92(\mathrm{t}, J=7.5 \mathrm{~Hz}, 3 \mathrm{H}$, $\left.\mathrm{CH}_{3} \mathrm{CH}_{2}\right), 0.91\left(\mathrm{~s}, 9 \mathrm{H}, \mathrm{CMe}_{3}\right), 0.09\left(\mathrm{~s}, 3 \mathrm{H}, \mathrm{SiCH}_{3}\right), 0.08(\mathrm{~s}, 3 \mathrm{H}$, $\left.\mathrm{SiCH}_{3}\right) ;{ }^{13} \mathrm{C}\left\{{ }^{1} \mathrm{H}\right\}$ NMR $\left(100 \mathrm{MHz}, \mathrm{CDCl}_{3}\right) \delta 159.2(\mathrm{Ar}), 137.6(\mathrm{Ar})$, 130.4 (Ar), 129.6 (Ar), 128.5 (Ar), 127.9 (Ar), $127.6(\mathrm{Ar}), 113.8$ (Ar), 81.1(CHOPMB), 80.3 (CHORCHOH), 80.2 $\left(\mathrm{CHORCH}_{2} \mathrm{OH}\right), 79.6$ (CHOBn), 75.6 (CHOTBS), 71.6 $(\mathrm{CHOH}), 71.6\left(\mathrm{CH}_{2} \mathrm{Ar}\right), 71.4\left(\mathrm{CH}_{2} \mathrm{Ar}\right), 62.3\left(\mathrm{CH}_{2} \mathrm{OH}\right), 55.3$ (OMe), $33.4\left(\mathrm{CH}_{2} \mathrm{CHOBn}\right), 33.0\left(\mathrm{CH}_{2} \mathrm{CHOPMB}\right), 26.0\left(\mathrm{CMe}_{3}\right.$, $\left.\mathrm{CH}_{3} \mathrm{CH}_{2}\right), 18.2\left(\mathrm{CMe}_{3}\right), 10.6\left(\mathrm{CH}_{3} \mathrm{CH}_{2}\right),-4.1\left(\mathrm{SiCH}_{3}\right),-4.7$ $\left(\mathrm{SiCH}_{3}\right)$; HRMS (ESI-TOF) $m / z[\mathrm{M}+\mathrm{Na}]^{+}$calcd for $\mathrm{C}_{32} \mathrm{H}_{50} \mathrm{O}_{7} \mathrm{SiNa}$ 597.3218; found 597.3240; $[\alpha]_{\mathrm{D}}^{20}-13.0\left(c=1\right.$ in $\left.\mathrm{CHCl}_{3}\right)$.

$\left(\left(2 R, 2^{\prime} S, 4 R, 4^{\prime} S, 5 R, 5^{\prime} R\right)\right.$ - 4- (Benzyloxy) - $5^{\prime}$ - ethyl-4' - ((4methoxybenzyl)oxy)octahydro-[2,2'-bifuran $]-5-y l)$ methylmethanesulfonate (24). To a stirred solution of diol 21 (900 $\mathrm{mg}, 1.57 \mathrm{mmol})$ in $\mathrm{DCM}(30 \mathrm{~mL})$ at $0{ }^{\circ} \mathrm{C}$ were added ethyldiisopropylamine $(2.72 \mathrm{~mL}, 15.6 \mathrm{mmol})$ and $\mathrm{MsCl}(1 \mathrm{~mL}$, $12.5 \mathrm{mmol}$ ) and stirred for $1 \mathrm{~h}$ before being quenched with saturated aqueous $\mathrm{NH}_{4} \mathrm{Cl}(30 \mathrm{~mL})$ and diluted with $\mathrm{H}_{2} \mathrm{O}(30 \mathrm{~mL})$ and DCM $(30 \mathrm{~mL})$. The aqueous layer was separated and extracted with DCM $(3 \times 30 \mathrm{~mL})$. The combined organic layers were dried $\left(\mathrm{Na}_{2} \mathrm{SO}_{4}\right)$, filtered, and concentrated in vacuo to give a crude oil (23). The crude oil was dissolved in $\mathrm{DCM} / \mathrm{MeOH}(1: 1,30 \mathrm{~mL})$; then CSA $(363 \mathrm{mg}$, $1.57 \mathrm{mmol}$ ) was added, and the reaction was stirred for $24 \mathrm{~h}$ before being quenched by the addition of saturated aqueous $\mathrm{NaHCO}_{3}(40$ $\mathrm{mL})$. The aqueous layer was separated and extracted with DCM $(3 \times$ $30 \mathrm{~mL})$. The combined organic layers were dried $\left(\mathrm{Na}_{2} \mathrm{SO}_{4}\right)$, filtered, and concentrated in vacuo to give a crude oil. The crude oil was then dissolved in ${ }^{t} \mathrm{BuOH}(20 \mathrm{~mL})$ and warmed to $35^{\circ} \mathrm{C}$; ${ }^{t} \mathrm{BuOK}(527 \mathrm{mg}$, $4.71 \mathrm{mmol}$ ) was added and the reaction stirred for $1 \mathrm{~h}$. The reaction was quenched with saturated aqueous $\mathrm{NH}_{4} \mathrm{Cl}(30 \mathrm{~mL})$. The aqueous layer was separated and extracted with EtOAc $(3 \times 30 \mathrm{~mL})$. The combined organic layers were dried $\left(\mathrm{MgSO}_{4}\right)$, filtered, and concentrated in vacuo. Purification via flash column chromatography ( $2: 1 \mathrm{petrol} /$ ethyl acetate) gave the title compound as a colorless oil $(427 \mathrm{mg}, 0.82 \mathrm{mmol}, 52 \%): R_{f} 0.56\left(2: 1 \mathrm{petrol} /\right.$ ethyl acetate); $\nu_{\max } /$ $\mathrm{cm}^{-1}$ (thin film) 2935m, 1356s, $1175 \mathrm{~s} ;{ }^{1} \mathrm{H}$ NMR (400 $\mathrm{MHz} \mathrm{CDCl}_{3}$ ) $\delta 7.22-7.38(\mathrm{~m}, 7 \mathrm{H}, \mathrm{ArH}), 6.88(\mathrm{~d}, J=8.6 \mathrm{~Hz}, 2 \mathrm{H}, \mathrm{ArH}), 4.61(\mathrm{~d}, J=$ $\left.11.8 \mathrm{~Hz}, 1 \mathrm{H}, \mathrm{CHH}^{\prime} \mathrm{Ar}\right), 4.36\left(\mathrm{~d}, J=11.9 \mathrm{~Hz}, 1 \mathrm{H}, \mathrm{CHH}^{\prime} \mathrm{Ar}\right), 4.32-$ $4.52\left(\mathrm{~m}, 5 \mathrm{H}, 2 \times \mathrm{CHH}^{\prime} \mathrm{Ar}, \mathrm{CHORCH}_{2} \mathrm{OMs}\right), 4.12-422(\mathrm{~m}, 2 \mathrm{H}$, CHOBn, CHOPMB), 4.00-4.09 (m, 2H, CHORCHOR), 3.85-3.92 (m, 1H, EtCHOR), 3.81 (s, 3H, OMe), 3.00 (s, 3H, SMe), 2.21-2.30 $\left(\mathrm{m}, 2 \mathrm{H}, 2 \times \mathrm{CHH}^{\prime} \mathrm{CHOR}\right), 2.16(\mathrm{dt}, J=13.1,4.4 \mathrm{~Hz}, 1 \mathrm{H}$, $\left.\mathrm{CHH}^{\prime} \mathrm{CHOBn}\right), 2.00\left(\mathrm{dt}, J=12.6,4.4 \mathrm{~Hz}, 1 \mathrm{H}, \mathrm{CHOPMBCHH}^{\prime}\right)$, 1.48 (qn, $\left.J=7.6 \mathrm{~Hz}, 2 \mathrm{H}, \mathrm{CH}_{3} \mathrm{CH}_{2}\right), 0.93(\mathrm{t}, J=7.6 \mathrm{~Hz}, 3 \mathrm{H}$, $\left.\mathrm{CH}_{3} \mathrm{CH}_{2}\right) ;{ }^{13} \mathrm{C}\left\{{ }^{1} \mathrm{H}\right\}$ NMR $\left(100 \mathrm{MHz}, \mathrm{CDCl}_{3}\right) \delta 159.2(\mathrm{Ar}), 137.5$ (Ar), $130.2(\mathrm{Ar}), 129.3(\mathrm{Ar}), 128.5(\mathrm{Ar}), 127.9(\mathrm{Ar}), 127.8(\mathrm{Ar})$, 113.8 (Ar), 84.9 (EtCHOR), 82.4 (CHOPMB), 80.9 (CHOBn), 79.8 (CHORCHOR), 79.3 (CHORCHOR), 78.7 ( $\left.\mathrm{CH}_{2} \mathrm{OMs}\right), 77.3$ 
( $\left.\mathrm{CHORCH}_{2} \mathrm{OMs}\right), 71.1\left(\mathrm{CH}_{2} \mathrm{Ar}\right), 69.8\left(\mathrm{CH}_{2} \mathrm{Ar}\right), 55.3(\mathrm{OMe}), 37.8$ $\left(\mathrm{SCH}_{3}\right), 34.6\left(\mathrm{CH}_{2}\right), 34.1\left(\mathrm{CH}_{2}\right), 26.7\left(\mathrm{CH}_{3} \mathrm{CH}_{2}\right), 10.1\left(\mathrm{CH}_{3}\right) ; \mathrm{MS}$ (ESI-TOF) $m / z 543[\mathrm{M}+\mathrm{Na}]^{+}$; HRMS (ESI-TOF) $m / z[\mathrm{M}+\mathrm{Na}]^{+}$ calcd for $\mathrm{C}_{27} \mathrm{H}_{36} \mathrm{O}_{8} \mathrm{SNa} 543.2023$; found 543.2022; $[\alpha]_{\mathrm{D}}^{20}+4.0(c=$ 1.0 in $\left.\mathrm{CHCl}_{3}\right)$.

$((2 R, 3 R, 5 R)-3-($ Benzyloxy)-5-((1R,3S,4R)-4-((tert-butyldimethylsilyl)oxy)-1-hydroxy-3-((4-methoxybenzyl)oxy)hexyl)tetrahydrofuran-2-yl)methylmethanesulfonate (22). The title compound was isolated as a side product in the synthesis of $24: R_{f} 0.63$ (2:1 petrol/ ethyl acetate); $\nu_{\max } / \mathrm{cm}^{-1}$ (thin film) $3540 \mathrm{br}, 2931 \mathrm{~m}, 1356 \mathrm{~s}, 1174 \mathrm{~s}$; ${ }^{1} \mathrm{H}$ NMR (400 MHz CDCl $\left.{ }_{3}\right) \delta 7.25-7.37(\mathrm{~m}, 7 \mathrm{H}, \mathrm{ArH}), 6.88(\mathrm{~d}, J=$ $8.1 \mathrm{~Hz}, 2 \mathrm{H}, \mathrm{ArH}), 4.69$ (d, $\left.J=11.0 \mathrm{~Hz}, 1 \mathrm{H}, \mathrm{CHH}^{\prime} \mathrm{Ar}\right), 4.57(\mathrm{~d}, J=$ $\left.11.7 \mathrm{~Hz}, 1 \mathrm{H}, \mathrm{CHH}^{\prime} \mathrm{Ar}\right), 4.43-4.46\left(\mathrm{~m}, 2 \mathrm{H}, \mathrm{CH}_{2} \mathrm{OMs}\right), 4.41$ (d, $J=$ $\left.11.1 \mathrm{~Hz}, 1 \mathrm{H}, \mathrm{CHH}^{\prime} \mathrm{Ar}\right), 4.36$ (d, $\left.J=11.7 \mathrm{~Hz}, 1 \mathrm{H}, \mathrm{CHH}^{\prime} \mathrm{Ar}\right), 4.19$ (q, $\left.J=5.3 \mathrm{~Hz}, 1 \mathrm{H}, \mathrm{CHORCH}_{2} \mathrm{OMs}\right), 4.12(\mathrm{q}, J=5.3 \mathrm{~Hz}, 1 \mathrm{H}, \mathrm{CHOBn})$, $3.91(\mathrm{q}, J=5.0 \mathrm{~Hz}, 1 \mathrm{H}, \mathrm{CHOHCHOR}), 3.80(\mathrm{~s}, 4 \mathrm{H}, \mathrm{OMe}, \mathrm{OH})$, 3.75 (ddd, $J=7.3,4.9,2.0 \mathrm{~Hz}, 1 \mathrm{H}, \mathrm{CHOTBS}), 3.62(\mathrm{td}, J=6.0,2.0$ $\mathrm{Hz}, 1 \mathrm{H}, \mathrm{CHOPMB}), 2.99$ (s, 3H, SMe), 2.07-2.18 (m, 1H, CHHCHOBn), 1.98 (ddd, $J=13.1,7.6,4.4 \mathrm{~Hz}, 1 \mathrm{H}, \mathrm{CHHCHOBn}$ ), 1.71-1.75 (m, 2H, $\left.\mathrm{CH}_{2} \mathrm{CHOPMB}\right), 1.50-1.61\left(\mathrm{~m}, 1 \mathrm{H}, \mathrm{CH}_{3} \mathrm{CHH}\right)$, $1.49-1.39\left(\mathrm{~m}, 1 \mathrm{H}, \mathrm{CH}_{3} \mathrm{CHH}\right), 0.92\left(\mathrm{t}, J=7.6 \mathrm{~Hz}, 3 \mathrm{H}, \mathrm{CH}_{3} \mathrm{CH}_{2}\right)$, $0.91\left(\mathrm{~s}, 9 \mathrm{H}, \mathrm{CMe}_{3}\right), 0.09\left(\mathrm{~s}, 3 \mathrm{H}, \mathrm{SiCH}_{3}\right), 0.08\left(\mathrm{~s}, 3 \mathrm{H}, \mathrm{SiCH}_{3}\right)$; ${ }^{13} \mathrm{C}\left\{{ }^{1} \mathrm{H}\right\}$ NMR $\left(100 \mathrm{MHz}, \mathrm{CDCl}_{3}\right) \delta 159.2(\mathrm{Ar}), 137.4$ (Ar), 130.4 (Ar), 129.6 (Ar) 128.5 (Ar), 128.0 (Ar), 127.7 (Ar), $113.8(\mathrm{Ar}), 80.9$ (CHORCHOH), 80.8 (CHOPMB), 80.6 (CHOBn), 78.7 (CHORCH $\mathrm{CHMs}_{2} \mathrm{OM}, 78.4$ ( $\left.\mathrm{CH}_{2} \mathrm{OMs}\right), 75.5$ (CHOTBS), 71.4 $\left(\mathrm{CH}_{2} \mathrm{Ar}\right), 71.0(\mathrm{CHOH}), 69.4\left(\mathrm{CH}_{2} \mathrm{Ar}\right), 55.3(\mathrm{OMe}), 37.5$ $\left(\mathrm{CH}_{2} \mathrm{CHOBn}\right), 32.6\left(\mathrm{CH}_{2} \mathrm{CHOPMB}\right), 26.1\left(\mathrm{CH}_{3} \mathrm{CH}_{2}\right), 26.0$ $\left(\mathrm{CMe}_{3}\right), 18.2\left(\mathrm{CMe}_{3}\right), 10.9\left(\mathrm{CH}_{3} \mathrm{CH}_{2}\right),-4.1 \quad(\mathrm{SiMe}),-4.7$ $(\mathrm{SiMe}) ; \mathrm{MS}(\mathrm{ESI}-\mathrm{TOF}) \mathrm{m} / z 675[\mathrm{M}+\mathrm{Na}]^{+}$; HRMS (ESI-TOF) $m / z[\mathrm{M}+\mathrm{Na}]^{+}$calcd for $\mathrm{C}_{33} \mathrm{H}_{52} \mathrm{O}_{9} \mathrm{SiSNa}$ 675.2994; found 675.2990; $[\alpha]_{\mathrm{D}}^{20}-55.6\left(c=1.0\right.$ in $\left.\mathrm{CHCl}_{3}\right)$.

(2R,2'S,4R,4'S,5S,5'R)-4-(Benzyloxy)-5' -ethyl-5-(iodomethyl)-4' ((4-methoxybenzyl)oxy)octahydro-2,2'-bifuran (25). TBAI (3 g, 8.2 $\mathrm{mmol})$ and mesylate $24(420 \mathrm{mg}, 0.82 \mathrm{mmol})$ were dissolved in dry toluene $(6 \mathrm{~mL})$ and heated to $110^{\circ} \mathrm{C}$ for $16 \mathrm{~h}$ with vigorous stirring. The reaction mixture was cooled to rt, and then filtered through a sinter washing with diethyl ether $(100 \mathrm{~mL})$. The filtrate was concentrated in vacuo and purified by flash column chromatography ( $8: 1$ petrol/ethyl acetate) to give the title compound as a colorless oil (360 mg, $0.65 \mathrm{mmol}, 80 \%)$ and recovered SM $(40 \mathrm{mg}, 0.08 \mathrm{mmol}$, 9\%): $R_{f} 0.47$ (10:1 petrol/ethyl acetate); $\nu_{\max } / \mathrm{cm}^{-1}$ (thin film) 2961m; ${ }^{1} \mathrm{H}$ NMR (400 MHz CDCl $\left.{ }_{3}\right) \delta 7.23-7.39$ (m, 7H, ArH), 6.86-6.90 (m, 2H, ArH), 4.63 (d, $\left.J=11.4 \mathrm{~Hz}, 1 \mathrm{H}, \mathrm{CHH} \mathrm{H}^{\prime} \mathrm{Ar}\right), 4.49$ (d, $\left.J=11.4 \mathrm{~Hz}, 1 \mathrm{H}, \mathrm{CHH}^{\prime} \mathrm{Ar}\right), 4.43$ (d, $J=11.4 \mathrm{~Hz}, 1 \mathrm{H}, \mathrm{CHH}^{\prime} \mathrm{Ar}$ ), $4.38(\mathrm{~d}, J=11.4 \mathrm{~Hz}, 1 \mathrm{H}, \mathrm{CHH} \mathrm{Ar}), 4.17(\mathrm{td}, J=4.6,2.8 \mathrm{~Hz}, 2 \mathrm{H}$, CHORCHOR), $4.08-4.15$ (m, 2H, CHOBnCHOR), 3.99 (td, $J=$ 7.3, $5.0 \mathrm{~Hz}, 1 \mathrm{H}$, CHORCHOR), $3.88(\mathrm{td}, J=6.6,3.7 \mathrm{~Hz}, 1 \mathrm{H}$, CHOPMB), 3.81 (s, 3H, OMe), $3.79(\mathrm{dt}, J=6.4,3.5 \mathrm{~Hz}, 1 \mathrm{H}), 3.41$ $\left(\mathrm{dd}, J=9.4,8.3 \mathrm{~Hz}, 1 \mathrm{H}, \mathrm{CH} \mathrm{H}^{\prime} \mathrm{I}\right), 3.28(\mathrm{dt}, J=9.3,5.8 \mathrm{~Hz}, 1 \mathrm{H}$, $\left.\mathrm{CHH}^{\prime} \mathrm{I}\right), 2.18-2.33\left(\mathrm{~m}, 3 \mathrm{H}, \mathrm{CH}_{2} \mathrm{CHOBn}, \mathrm{CHH}^{\prime} \mathrm{CHOPMB}\right), 2.03$ (ddd, $\left.J=13.2,7.3,3.0 \mathrm{~Hz}, 1 \mathrm{H}, \mathrm{CH} \mathrm{H}^{\prime} \mathrm{CHOPMB}\right), 1.52-1.42(\mathrm{~m}, 2 \mathrm{H}$, $\left.\mathrm{CH}_{3} \mathrm{CH}_{2}\right), 0.93\left(\mathrm{t}, J=7.0 \mathrm{~Hz}, 3 \mathrm{H}, \mathrm{CH}_{3}\right) ;{ }^{13} \mathrm{C}\left\{{ }^{1} \mathrm{H}\right\} \mathrm{NMR}(100 \mathrm{MHz}$, $\mathrm{CDCl}_{3}$ ) $\delta 159.2(\mathrm{Ar}), 138.0(\mathrm{Ar}), 130.2(\mathrm{Ar}), 129.3(\mathrm{Ar}), 128.3(\mathrm{Ar})$, 127.9 (Ar), 127.7 (Ar), 113.8 (Ar), 84.8 (EtCHOR), 83.0 (CHOBn), 82.5 (CHOPMB), $81.3\left(\mathrm{CHORCH}_{2} \mathrm{I}\right), 80.1$ (CHORCHOR), 78.6 (CHORCHOR), $71.3\left(\mathrm{CH}_{2} \mathrm{Ar}\right), 70.8\left(\mathrm{CH}_{2} \mathrm{Ar}\right), 55.3(\mathrm{OMe}), 34.6$ $\left(\mathrm{CH}_{2} \mathrm{CHOBn}\right), 34.1\left(\mathrm{CH}_{2} \mathrm{CHOPMB}\right), 26.7\left(\mathrm{CH}_{3} \mathrm{CH}_{2}\right), 10.1\left(\mathrm{CH}_{3}\right)$, $2.2\left(\mathrm{CH}_{2} \mathrm{I}\right)$; MS (ESI-TOF) $m / z 575[\mathrm{M}+\mathrm{Na}]^{+}$; HRMS (ESI-TOF) $m / z[\mathrm{M}+\mathrm{Na}]^{+}$calcd for $\mathrm{C}_{26} \mathrm{H}_{33} \mathrm{O}_{5} \mathrm{INa} 575.1265$; found 575.1283; $[\alpha]_{\mathrm{D}}^{20}-16.5\left(c=1.0\right.$ in $\left.\mathrm{CHCl}_{3}\right)$.

$\left(2 R, 2^{\prime} S, 4 R, 4^{\prime} S, 5 R, 5^{\prime} R\right)$-5-Allyl-4-(benzyloxy)-5' -ethyl-4' -((4methoxybenzyl)oxy)octahydro-2,2' -bifuran (11). Iodide 25 (360 $\mathrm{mg}, 0.65 \mathrm{mmol}$ ) was dried by being azeotroped with dry benzene three times and then dissolved in dry benzene $(13 \mathrm{~mL})$ and warmed to $40{ }^{\circ} \mathrm{C}$. Vinylmagnesium bromide $(13 \mathrm{~mL}, 1 \mathrm{M}$ in THF, $13 \mathrm{mmol})$ was added and the reaction stirred for $3 \mathrm{~h}$. The reaction mixture was cooled to $0{ }^{\circ} \mathrm{C}$ and then quenched with dropwise addition of saturated aqueous $\mathrm{NH}_{4} \mathrm{Cl}(20 \mathrm{~mL})$ and diluted with $\mathrm{H}_{2} \mathrm{O}(10 \mathrm{~mL})$ and EtOAc $(10 \mathrm{~mL})$. The aqueous layer was separated and extracted
$(3 \times 30 \mathrm{~mL}$ EtOAc). The combined organic layers were dried $\left(\mathrm{MgSO}_{4}\right)$, filtered, and concentrated in vacuo. Purification by flash column chromatography $(50: 1 \rightarrow 25: 1 \mathrm{DCM} /$ ethyl acetate $)$ gave the title compound as a colorless oil $(166 \mathrm{mg}, 0.37 \mathrm{mmol}, 57 \%): R_{f} 0.45$ (10:1 petrol/ethyl acetate); $\nu_{\max } / \mathrm{cm}^{-1}$ (thin film) $2933 \mathrm{~m} ;{ }^{1} \mathrm{H}$ NMR $\left(400 \mathrm{MHz} \mathrm{CDCl}_{3}\right) \delta 7.23-7.39(\mathrm{~m}, 7 \mathrm{H}, \mathrm{ArH}), 6.86-6.90(\mathrm{~m}, 2 \mathrm{H}$, $\mathrm{ArH}), 5.86\left(\mathrm{ddt}, J=17.0,10.2,7.7 \mathrm{~Hz}, 1 \mathrm{H}, \mathrm{CH}=\mathrm{CH}_{2}\right), 5.12(\mathrm{dq}, J=$ $\left.17.0,2.0 \mathrm{~Hz}, 1 \mathrm{H}, \mathrm{CH}=\mathrm{CHH}^{\prime}\right), 5.04(\mathrm{ddt}, J=10.2,2.0,1.2 \mathrm{~Hz}, 1 \mathrm{H}$, $\left.\mathrm{CH}=\mathrm{CHH}^{\prime}\right), 4.62\left(\mathrm{~d}, J=11.9 \mathrm{~Hz}, 1 \mathrm{H}, \mathrm{CHH}^{\prime} \mathrm{Ar}\right), 4.49(\mathrm{~d}, J=11.9$ $\mathrm{Hz}, 1 \mathrm{H}, \mathrm{CHH}^{\prime} \mathrm{Ar}$ ), 4.38 (d, $J=11.9 \mathrm{~Hz}, 2 \mathrm{H}, \mathrm{CH} \mathrm{H}^{\prime} \mathrm{Ar}$ ), 3.93-4.03 (m, 2H, CHOBn, CHORCHOR), $3.88(\mathrm{td}, J=6.5,3.9 \mathrm{~Hz}, 1 \mathrm{H}$, EtCHOR), $3.81(\mathrm{~s}, 3 \mathrm{H}, \mathrm{OMe}), 3.76-3.80(\mathrm{~m}, 3 \mathrm{H}, \mathrm{CHOPMB}$, CHORCHOR, CHOBnCHOR), 2.42-2.57 (m, 2H, $\mathrm{CH}_{2} \mathrm{CH}=$ $\left.\mathrm{CH}_{2}\right), 2.18-2.34\left(\mathrm{~m}, 2 \mathrm{H}, 2 \times \mathrm{CHH}^{\prime} \mathrm{CHOR}\right), 2.11$ (ddd, $J=13.7$, 4.9, $\left.2.3 \mathrm{~Hz}, 1 \mathrm{H}, \mathrm{CH} \mathrm{H}^{\prime} \mathrm{CHOPMB}\right), 2.06(\mathrm{dt}, J=13.4,5.2 \mathrm{~Hz}, 1 \mathrm{H}$, $\left.\mathrm{CH} H^{\prime} \mathrm{CHOBn}\right), 1.45-1 / 52\left(\mathrm{~m}, 2 \mathrm{H}, \mathrm{CH}_{3} \mathrm{CH}_{2}\right), 0.93(\mathrm{t}, J=7.5 \mathrm{~Hz}$, $\left.3 \mathrm{H}, \mathrm{CH}_{3}\right) ;{ }^{13} \mathrm{C}\left\{{ }^{1} \mathrm{H}\right\}$ NMR $\left(100 \mathrm{MHz}, \mathrm{CDCl}_{3}\right) \delta 159.2(\mathrm{Ar}) 138.4$ (Ar), $135.4\left(\mathrm{CH}=\mathrm{CH}_{2}\right), 130.3(\mathrm{Ar}), 129.3(\mathrm{Ar}), 128.3(\mathrm{Ar}), 127.6$ (Ar), $127.5(\mathrm{Ar}), 116.6\left(\mathrm{CH}=\mathrm{CH}_{2}\right), 113.8(\mathrm{Ar}), 84.6(\mathrm{EtCHOR})$, 82.5, 82.3, 80.4 (CHOPMB, CHORCHOR, CHORCHOPMB), 80.0, 78.8 (CHORCHOR, CHOBn), $70.8\left(\mathrm{CH}_{2} \mathrm{Ar}\right), 70.7\left(\mathrm{CH}_{2} \mathrm{Ar}\right), 55.3$ (OMe), $34.8\left(\mathrm{CH}_{2} \mathrm{CHOPMB}\right), 34.6\left(\mathrm{CH}_{2} \mathrm{COBn}\right), 33.9\left(\mathrm{CH}_{2} \mathrm{CH}=\right.$ $\left.\mathrm{CH}_{2}\right), 26.7\left(\mathrm{CH}_{3} \mathrm{CH}_{2}\right), 10.1\left(\mathrm{CH}_{3}\right)$; MS (ESI-TOF) $m / z 475[\mathrm{M}+$ $\mathrm{Na}]^{+}$; HRMS (ESI-TOF) $m / z[\mathrm{M}+\mathrm{Na}]^{+}$calcd for $\mathrm{C}_{28} \mathrm{H}_{36} \mathrm{O}_{5} \mathrm{Na}$ 475.2455; found 475.2440; $[\alpha]_{\mathrm{D}}^{20}-6.4\left(c=1.0\right.$ in $\left.\mathrm{CHCl}_{3}\right)$.

(2R, 2'S, 4R, 4'S,5R,5'R)-5-Allyl-5'-ethyl-4'-((4-methoxybenzyl)oxy)octahydro-[2,2'-bifuran]-4-ol (26). To a stirring solution of ether 11 (150 mg, $0.33 \mathrm{mmol})$ in THF $(12 \mathrm{~mL})$ at $-78^{\circ} \mathrm{C}$ was added dropwise LiDBB (2 mL of a solution of LIDBB prepared by sonicating DBB ( $1.0 \mathrm{~g}, 3.7 \mathrm{mmol})$ and lithium $(26 \mathrm{mg}, 3.7 \mathrm{mmol})$ in THF $(4 \mathrm{~mL})$ for $2 \mathrm{~h}$ ). Reaction progress was monitored by TLC every $0.4 \mathrm{~mL}$ of LiDBB solution. When no starting material was detected, the reaction was quenched with saturated aqueous $\mathrm{NH}_{4} \mathrm{Cl}$ $(10 \mathrm{~mL})$, diluted with EtOAc $(10 \mathrm{~mL})$, and warmed to $\mathrm{rt}$. The aqueous layer was separated and extracted with EtOAc $(3 \times 15 \mathrm{~mL})$. The combined organic layers were dried $\left(\mathrm{MgSO}_{4}\right)$, filtered, and concentrated in vacuo. Purification by flash column chromatography (5:1 petrol/ethyl acetate) gave the title compound as a colorless oil (92 mg, $2.5 \mathrm{mmol}, 77 \%): R_{f} 0.34$ (5:1 petrol/ethyl acetate); $\nu_{\max } /$ $\mathrm{cm}^{-1}$ (thin film) 3413br, 2934; ${ }^{1} \mathrm{H}$ NMR $\left(400 \mathrm{MHz} \mathrm{CDCl}_{3}\right) \delta 7.23-$ $7.28(\mathrm{~m}, 2 \mathrm{H}, \mathrm{ArH}), 6.87-6.91(\mathrm{~m}, 2 \mathrm{H}, \mathrm{ArH}), 5.88$ (ddt, $J=17.4$, $\left.10.2,7.0 \mathrm{~Hz}, 1 \mathrm{H}, \mathrm{CH}=\mathrm{CH}_{2}\right), 5.16(\mathrm{dq}, J=17.4,1.5 \mathrm{~Hz}, 1 \mathrm{H}, \mathrm{CH}=$ $\left.\mathrm{CHH}^{\prime}\right), 5.07\left(\mathrm{ddt}, J=10.2,2.1,1.5 \mathrm{~Hz}, 1 \mathrm{H}, \mathrm{CH}=\mathrm{CHH}^{\prime}\right), 4.46(\mathrm{~d}, J$ $\left.=11.4 \mathrm{~Hz}, 1 \mathrm{H}, \mathrm{CHH}^{\prime} \mathrm{Ar}\right), 4.43\left(\mathrm{~d}, J=11.4 \mathrm{~Hz}, 1 \mathrm{H}, \mathrm{CHH} \mathrm{Hr}^{\prime}\right), 4.23$ (ddd, $J=9.4,7.1,1.8 \mathrm{~Hz}, 1 \mathrm{H}, \mathrm{CHORCHOR}), 4.16-4.08(\mathrm{~m}, 2 \mathrm{H}$, CHORCHOR, OH), $4.00($ br s, $1 \mathrm{H}, \mathrm{CHOH}), 3.91(\mathrm{dt}, J=7.7,5.2$ $\mathrm{Hz}, 1 \mathrm{H}, \mathrm{EtCHOR}), 3.79-3.83$ (m, 4H, OMe, CHOPMB), 3.66 (td, $J$ $=7.0,2.4 \mathrm{~Hz}, 1 \mathrm{H}, \mathrm{CHORCHOH}), 2.54-2.37\left(\mathrm{~m}, 2 \mathrm{H}, \mathrm{CH}_{2} \mathrm{CH}=\right.$ $\left.\mathrm{CH}_{2}\right), 2.30\left(\mathrm{dt}, \mathrm{J}=12.7,6.9 \mathrm{~Hz}, 1 \mathrm{H}, \mathrm{CHH}^{\prime} \mathrm{CHOPMB}\right) 2.21$ (ddd, $\left.1 \mathrm{H}, J=14.0,10.0,5.2 \mathrm{~Hz}, \mathrm{CHH}^{\prime} \mathrm{CHOH}\right), 2.14(\mathrm{td}, J=14.0,3.9 \mathrm{~Hz}$, $\left.\mathrm{CHH}{ }^{\prime} \mathrm{CHOH}\right), 1.59-1.51\left(3 \mathrm{H}, \mathrm{m}, \mathrm{CHH}^{\prime} \mathrm{CHOPMB}, \mathrm{CH}_{3} \mathrm{CH}_{2}\right), 0.96$ $\left(\mathrm{t}, J=7.5 \mathrm{~Hz}, 3 \mathrm{H}, \mathrm{CH}_{3}\right) ;{ }^{13} \mathrm{C}\left\{{ }^{1} \mathrm{H}\right\} \mathrm{NMR}\left(100 \mathrm{MHz}, \mathrm{CDCl}_{3}\right) \delta 159.3$ $(\mathrm{Ar}), 135.2\left(\mathrm{CH}=\mathrm{CH}_{2}\right), 129.9(\mathrm{Ar}), 129.2(\mathrm{Ar}), 116.7\left(\mathrm{CH}=\mathrm{CH}_{2}\right)$, 133.9 (Ar), 85.0 (EtCHOR), 83.8 (CHORCHOH), 81.8 (CHOPMB), 79.7 (CHORCHOR), 78.5 (CHORCHOR), 71.5 $\left(\mathrm{CH}_{2} \mathrm{Ar}\right), 70.9(\mathrm{CHOH}), 55.3(\mathrm{OMe}), 34.9\left(\mathrm{CH}_{2} \mathrm{CHOPMB}\right), 34.2$ $\left(\mathrm{CH}_{2} \mathrm{CHOH}\right), 33.6\left(\mathrm{CH}_{2} \mathrm{CH}=\mathrm{CH}_{2}\right), 26.4\left(\mathrm{CH}_{3} \mathrm{CH}_{2}\right), 10.3\left(\mathrm{CH}_{3}\right)$; MS (ESI-TOF) $m / z 385[\mathrm{M}+\mathrm{Na}]^{+}$; HRMS (ESI-TOF) $m / z[\mathrm{M}+$ $\mathrm{Na}]^{+}$calcd for $\mathrm{C}_{21} \mathrm{H}_{30} \mathrm{O}_{5} \mathrm{Na} 385.1985$; found 385.1987; $[\alpha]_{\mathrm{D}}^{20}+18.9(c$ $=1.0$ in $\mathrm{CHCl}_{3}$ ).

$\left(2 R, 2^{\prime} S, 4 R, 4^{\prime} S, 5 R, 5^{\prime} R\right)-5$-Allyl-5' -ethyloctahydro-[2,2' -bifuran]$4,4^{\prime}$-diol (27). Isolated as a side product during the synthesis of $26: R_{f}$ 0.40 (ethyl acetate); $\nu_{\max } / \mathrm{cm}^{-1}$ (thin film) 3394br, 2935m; ${ }^{1} \mathrm{H}$ NMR $\left(500 \mathrm{MHz} \mathrm{CDCl}_{3}\right) \delta 5.87(\mathrm{ddt} J=17.0,10.0,7.0 \mathrm{~Hz}, 1 \mathrm{H}, \mathrm{CH}=$ $\left.\mathrm{CH}_{2}\right), 5.18\left(\mathrm{dq}, J=17.0,1.6 \mathrm{~Hz}, 1 \mathrm{H}, \mathrm{CH}=\mathrm{CHH}^{\prime}\right), 5.09(\mathrm{ddt}, J=$ 10.0, $\left.2.11 .6 \mathrm{~Hz}, 1 \mathrm{H}, \mathrm{CH}=\mathrm{CHH}^{\prime}\right), 4.26(\mathrm{ddd}, J=8.6,7.3,1.8 \mathrm{~Hz}$, $1 \mathrm{H}$, CHORCHOR $), 4.12-4.17(\mathrm{~m}, 2 \mathrm{H}$, CHORCHOR, $\left.\mathrm{CH}_{2} \mathrm{CHOH}\right), 4.07\left(\mathrm{dt}, J=6.3,4.3 \mathrm{~Hz}, 1 \mathrm{H}, \mathrm{CHOHCH}_{2}\right), 3.82$ (ddd, $J=9.2,6.3,4.3 \mathrm{~Hz}, 1 \mathrm{H}, \mathrm{EtCHOR}), 3.73(\mathrm{td}, J=6.9,2.7 \mathrm{~Hz}$, $1 \mathrm{H}, \mathrm{CHOHCHOR}), 2.49\left(\mathrm{tq}, J=6.9,1.6 \mathrm{~Hz}, 2 \mathrm{H}, \mathrm{CH}_{2} \mathrm{CH}=\mathrm{CH}_{2}\right)$, 
$2.25-2.33\left(\mathrm{~m}, 2 \mathrm{H}, 2 \times \mathrm{CHOHCHH}^{\prime}\right), 1.88(\mathrm{dd}, J=14.0,4.5 \mathrm{~Hz}$, $1 \mathrm{H}, \mathrm{CHOHCHH}$ ), 1.80 (ddd, $J=13.3,7.3,4.5 \mathrm{~Hz}, 1 \mathrm{H}$, $\left.\mathrm{CHH} H^{\prime} \mathrm{CHOH}\right), 1.42-1.55\left(\mathrm{~m}, 2 \mathrm{H}, \mathrm{CH}_{3} \mathrm{CH}_{2}\right), 0.98(\mathrm{t}, J=7.4 \mathrm{~Hz}$, $\left.3 \mathrm{H}, \mathrm{CH}_{3}\right) ;{ }^{13} \mathrm{C}\left\{{ }^{1} \mathrm{H}\right\}$ NMR $\left(125 \mathrm{MHz}, \mathrm{CDCl}_{3}\right) \delta 134.6\left(\mathrm{CH}=\mathrm{CH}_{2}\right)$, $117.1\left(\mathrm{CH}=\mathrm{CH}_{2}\right), 88.3(\mathrm{EtCHOR}), 83.4\left(\mathrm{CHORCH}_{2} \mathrm{CH}=\mathrm{CH}_{2}\right)$, 79.4 (CHORCHOR), 78.6 (CHORCHOR), $75.0\left(\mathrm{CHOHCH}_{2}\right), 71.3$ $\left(\mathrm{CH}_{2} \mathrm{CHOH}\right) 35.6\left(\mathrm{CH}_{2}\right), 35.4\left(\mathrm{CH}_{2}\right), 33.3\left(\mathrm{CH}_{2} \mathrm{CH}=\mathrm{CH}_{2}\right), 26.1$ $\left(\mathrm{CH}_{3} \mathrm{CH}_{2}\right), 10.2\left(\mathrm{CH}_{3}\right)$; MS (ESI-TOF) $\mathrm{m} / z 265[\mathrm{M}+\mathrm{Na}]^{+}$; HRMS (ESI-TOF) $m / z[\mathrm{M}+\mathrm{Na}]^{+}$calcd for $\mathrm{C}_{13} \mathrm{H}_{22} \mathrm{O}_{4} \mathrm{Na} 265.1410$; found 265.1413; $[\alpha]_{\mathrm{D}}^{20}+5.9\left(c=0.2\right.$ in $\left.\mathrm{CHCl}_{3}\right)$.

$\left(2 R, 2^{\prime} S, 4 S, 4^{\prime} S, 5 R, 5^{\prime} R\right)-5$-Allyl-4-chloro-5' -ethyl-4' -((4-methoxybenzyl)oxy)octahydro-2,2'-bifuran (28). To a stirred solution of alcohol 26 (86 mg, $0.24 \mathrm{mmol})$ and $\mathrm{PPh}_{3}(186 \mathrm{mg}, 0.70 \mathrm{mmol})$ in DCM $(6 \mathrm{~mL})$ was added $\mathrm{CCl}_{4}(1.5 \mathrm{~mL})$. The reaction mixture was stirred for $3 \mathrm{~h}$, and the yellow reaction mixture was loaded straight onto the column. Purification by flash column chromatography (DCM $\rightarrow$ 5:1 petrol/ethyl acetate) gave the title compound as a colorless oil (70 mg, $0.18 \mathrm{mmol}, 78 \%) ; R_{f} 0.89$ (5:1 petrol/ethyl acetate); $\nu_{\max } / \mathrm{cm}^{-1}$ (thin film) $2933 \mathrm{~m} ;{ }^{1} \mathrm{H}$ NMR (400 $\mathrm{MHz} \mathrm{CDCl}_{3}$ ) $\delta 7.26(\mathrm{~d}, J=8.3 \mathrm{~Hz}, 2 \mathrm{H}, \operatorname{ArH}), 6.89(\mathrm{~d}, J=8.3 \mathrm{~Hz}, 2 \mathrm{H}, \operatorname{ArH}), 5.85$ (ddt, $\left.J=17.2,10.1,6.8 \mathrm{~Hz}, 1 \mathrm{H}, \mathrm{CH}=\mathrm{CH}_{2}\right), 5.25-4.77(\mathrm{~m}, 2 \mathrm{H}$, $\left.\mathrm{CH}=\mathrm{CH}_{2}\right), 4.49\left(\mathrm{~d}, J=11.4 \mathrm{~Hz}, 1 \mathrm{H}, \mathrm{CHH}^{\prime} \mathrm{Ar}\right), 4.40(\mathrm{~d}, J=11.4 \mathrm{~Hz}$, $\left.1 \mathrm{H}, \mathrm{CHH} H^{\prime} \mathrm{Ar}\right), 4.22(\mathrm{q}, J=6.6 \mathrm{~Hz}, 1 \mathrm{H}, \mathrm{CHORCHOR}), 4.00-4.06$ $(\mathrm{m}, 2 \mathrm{H}, \mathrm{CHORCHCl}), 3.94(\mathrm{q}, J=6.6 \mathrm{~Hz}, 1 \mathrm{H}, \mathrm{CHORCHOR})$, 3.85-3.90(m, $1 \mathrm{H}, \mathrm{EtCHOR}), 3.81(\mathrm{OMe}), 3.78-3.82(\mathrm{~m}, 1 \mathrm{H}$, CHOPMB), 2.19-2.43 (m, 5H, $\mathrm{CH}_{2} \mathrm{CHOBn}, \mathrm{CH} H^{\prime} \mathrm{CHOPMB}$, $\left.\mathrm{CH}_{2} \mathrm{CH}=\mathrm{CH}_{2}\right), 1.93\left(\mathrm{dt}, J=12.3,6.6 \mathrm{~Hz}, 1 \mathrm{H}, \mathrm{CHH} H^{\prime} \mathrm{CHOPMB}\right)$, $1.49\left(\mathrm{qn}, J=7.6 \mathrm{~Hz}, 2 \mathrm{H}, \mathrm{CH}_{3} \mathrm{CH}_{2}\right), 0.93\left(\mathrm{t}, J=7.6 \mathrm{~Hz}, 3 \mathrm{H}, \mathrm{CH}_{3}\right)$; ${ }^{13} \mathrm{C}\left\{{ }^{1} \mathrm{H}\right\} \operatorname{NMR}\left(100 \mathrm{MHz}, \mathrm{CDCl}_{3}\right) \delta 159.2(\mathrm{Ar}), 133.6\left(\mathrm{CH}=\mathrm{CH}_{2}\right)$, $130.2(\mathrm{Ar}), 129.2(\mathrm{Ar}), 117.8(\mathrm{Ar}), 113.8\left(\mathrm{CH}=\mathrm{CH}_{2}\right), 113.7(\mathrm{Ar})$, 86.2 (CHORCHCl), 84.8 (EtCHOR), 82.2 (CHOPMB), 80.1 (CHORCHOR), 79.3 (CHORCHOR), $71.0\left(\mathrm{CH}_{2} \mathrm{Ar}\right), 59.2$ $(\mathrm{CHCl}), 55.3(\mathrm{OMe}), 38.1\left(\mathrm{CH}_{2} \mathrm{CH}=\mathrm{CH}_{2}\right), 37.9\left(\mathrm{CH}_{2} \mathrm{CHCl}\right)$, $34.6\left(\mathrm{CH}_{2} \mathrm{CHOPMB}\right), 26.6\left(\mathrm{CH}_{3} \mathrm{CH}_{2}\right), 10.1\left(\mathrm{CH}_{3}\right)$; MS (ESI-TOF) $\mathrm{m} / z 403\left[{ }^{35} \mathrm{M}+\mathrm{Na}\right]^{+}, 405\left[{ }^{37} \mathrm{M}+\mathrm{Na}\right]^{+}$; HRMS (ESI-TOF) $\mathrm{m} / z[\mathrm{M}$ $+\mathrm{Na}]^{+}$calcd for $\mathrm{C}_{21} \mathrm{H}_{29} \mathrm{O}_{4}{ }^{35} \mathrm{ClNa}$ 403.1647; found 403.1635; $[\alpha]_{\mathrm{D}}^{20}$ $+55.9\left(c=1.0\right.$ in $\left.\mathrm{CHCl}_{3}\right)$.

(E)-4- $\left(\left(2 R, 2^{\prime} S, 4 S, 4^{\prime} S, 5 R, 5^{\prime} R\right)\right.$-4-Chloro-5' - ethyl-4' - ((4methoxybenzyl)oxy)octahydro-[2,2'-bifuran]-5-yl)but-2-enal (30). To a stirred solution of alkene $28(62 \mathrm{mg}, 0.16 \mathrm{mmol})$ in dry degassed DCM $(4.5 \mathrm{~mL})$ were added crotonaldehyde $(134 \mu \mathrm{L}, 114$ $\mathrm{mg}, 1.6 \mathrm{mmol}$ ) and Grubbs' second generation catalyst $(14 \mathrm{mg}, 16$ $\mu \mathrm{mol})$. The reaction mixture was stirred for $1.5 \mathrm{~h}$ at $40{ }^{\circ} \mathrm{C}$ and then cooled to rt and quenched with the addition of DMSO $(0.1 \mathrm{~mL})$ and stirred for $16 \mathrm{~h}$. The mixture was concentrated in vacuo and purification by flash column chromatography (5:1 petrol ethyl acetate) gave the title compound as a colorless oil (58 mg, 14 mmol, 88\%): $R_{f} 0.25$ (5:1 petrol/ethyl acetate); $\nu_{\max } / \mathrm{cm}^{-1}$ (thin film) 2934m, 1691s; ${ }^{1} \mathrm{H}$ NMR (400 MHz $\left.\mathrm{CDCl}_{3}\right) \delta 9.52(\mathrm{~d}, J=7.8 \mathrm{~Hz}$, $1 \mathrm{H}, \mathrm{CHO}), 7.25(\mathrm{~d}, J=12.4 \mathrm{~Hz}, 2 \mathrm{H}, \mathrm{ArH}), 6.86-6.93(\mathrm{~m}, 3 \mathrm{H}, \mathrm{ArH}$, $\left.\mathrm{CH}_{2} \mathrm{CH}=\mathrm{CH}\right), 6.21(\mathrm{ddt} J=15.7,7.8,1.3 \mathrm{~Hz}, 1 \mathrm{H}, \mathrm{CHCHO}), 4.47$ $\left(\mathrm{d}, J=11.4 \mathrm{~Hz}, 1 \mathrm{H}, \mathrm{CH} \mathrm{H}^{\prime} \mathrm{Ar}\right), 4.41\left(\mathrm{~d}, J=11.4 \mathrm{~Hz}, 1 \mathrm{H}, \mathrm{CH} H^{\prime} \mathrm{Ar}\right)$, $4.24(\mathrm{td}, J=6.9,5.4 \mathrm{~Hz}, 1 \mathrm{H}, \mathrm{CHORCHOR}), 4.05$ (ddd, $J=7.2,5.8$, $4.5 \mathrm{~Hz}, 1 \mathrm{H}, \mathrm{CHORCHCl}), 4.01-3.96(\mathrm{~m}, 2 \mathrm{H}, \mathrm{CHCl}, \mathrm{CHORCH}-$ OR), 3.88 (td, $J=6.7,4.0 \mathrm{~Hz}, 1 \mathrm{H}, \mathrm{EtCHOR}), 3.79-3.83(\mathrm{~m}, 1 \mathrm{H}$, CHOPMB), 3.81 (s, 3H, OMe), 2.72 (dddd, $J=14.3,8.1,5.8,1.3 \mathrm{~Hz}$, $\left.1 \mathrm{H}, \mathrm{CHH}^{\prime} \mathrm{CH}=\mathrm{CH}\right), 2.58\left(\mathrm{dt}, J=14.3,5.8 \mathrm{~Hz}, 1 \mathrm{H}, \mathrm{CH} H^{\prime} \mathrm{CH}=\right.$ $\mathrm{CH}), 2.46\left(\mathrm{dt}, J=13.9,6.0 \mathrm{~Hz}, 1 \mathrm{H}, \mathrm{CHH}^{\prime} \mathrm{CHCl}\right), 2.30-2.19(\mathrm{~m}, 2 \mathrm{H}$, $\mathrm{CHH}{ }^{\prime} \mathrm{CHCl}, \mathrm{CH} H^{\prime} \mathrm{CHOH}$ ), 1.83 (ddd, $J=13.1,6.0,4.6 \mathrm{~Hz}, 1 \mathrm{H}$, $\mathrm{CH} \mathrm{H}^{\prime} \mathrm{CHOPMB}$ ), 1.48 (qn, $\left.J=7.6 \mathrm{~Hz}, 2 \mathrm{H}, \mathrm{CH}_{3} \mathrm{CH}_{2}\right), 0.93(\mathrm{t}, J=$ $\left.7.6 \mathrm{~Hz}, 3 \mathrm{H}, \mathrm{CH}_{3}\right) ;{ }^{13} \mathrm{C}\left\{{ }^{1} \mathrm{H}\right\}$ NMR $\left(100 \mathrm{MHz}, \mathrm{CDCl}_{3}\right) \delta 193.8$ $(\mathrm{CHO}), 159.3(\mathrm{Ar}), 153.1 \quad(\mathrm{CH}=\mathrm{CHCHO}), 135.1 \quad(\mathrm{CH}=$ CHCHO), $130.0(\mathrm{Ar}), 129.2(\mathrm{Ar}), 113.8(\mathrm{Ar}), 85.0(\mathrm{CHORCHCl})$, 84.7 (EtCHOR), 82.1 (CHOPMB), 80.4 (CHORCHOR), 78.9 (CHORCHOR), $71.1\left(\mathrm{CH}_{2} \mathrm{Ar}\right), 58.8(\mathrm{CHCl}), 55.3(\mathrm{OMe}), 37.3$ $\left(\mathrm{CH}_{2} \mathrm{CHCl}\right), 36.3\left(\mathrm{CH}_{2} \mathrm{CH}=\mathrm{CH}\right), 34.4\left(\mathrm{CH}_{2} \mathrm{CHOPMB}\right), \quad 26.5$ $\left(\mathrm{CH}_{3} \mathrm{CH}_{2}\right), 10.2\left(\mathrm{CH}_{3}\right) ; \mathrm{MS}$ (ESI-TOF) $\mathrm{m} / z 431\left[{ }^{35} \mathrm{M}+\mathrm{Na}\right]^{+}$, $433\left[{ }^{35} \mathrm{M}+\mathrm{Na}\right]^{+}$; HRMS (ESI-TOF) $m / z[\mathrm{M}+\mathrm{Na}]^{+}$calcd for $\mathrm{C}_{22} \mathrm{H}_{29} \mathrm{O}_{5}{ }^{35} \mathrm{ClNa} 431.1596$; found 431.1585; $[\alpha]_{\mathrm{D}}^{20}+22.8(c=1.0$ in $\mathrm{CHCl}_{3}$ ). $\left(2 R, 2^{\prime} S, 4 S, 4^{\prime} S, 5 R, 5^{\prime} R\right)-4-C h l o r o-5^{\prime}$-ethyl-4' -((4-methoxybenzyl)oxy)-5-((E)-pent-2-en-4-yn-1-yl)octahydro-2,2'-bifuran (31). To a solution of (diazomethyl)trimethylsilane $(50 \mu \mathrm{L}, 2 \mathrm{M}$ in ether, 0.1 $\mathrm{mmol})$ in THF at $-78{ }^{\circ} \mathrm{C}$ was added $\mathrm{BuLi}(62 \mu \mathrm{L}, 1.6 \mathrm{M}$ in hexanes, $0.1 \mathrm{mmol})$ dropwise. The reaction mixture was stirred for $30 \mathrm{~min}$ before being transferred rapidly to a solution of enal $30(4.2 \mathrm{mg}, 10$ $\mu \mathrm{mol})$ in THF $(0.5 \mathrm{~mL})$ at $-78{ }^{\circ} \mathrm{C}$. The reaction was stirred at -78 ${ }^{\circ} \mathrm{C}$ for $1 \mathrm{~h}$ before warming to $0{ }^{\circ} \mathrm{C}$ for a further $1 \mathrm{~h}$. The reaction was quenched with acetic acid $(0.1 \mathrm{~mL})$ and then saturated aqueous $\mathrm{NaHCO}_{3}(10 \mathrm{~mL})$. The aqueous layer was separated and extracted with EtOAc $(3 \times 10 \mathrm{~mL})$. The combined organic layers were dried $\left(\mathrm{MgSO}_{4}\right)$, filtered, and concentrated in vacuo. Purification by flash column chromatography (16:1 petrol/ethyl acetate) gave the title compound as a colorless oil $(2.9 \mathrm{mg}, 7 \mu \mathrm{mol}, 70 \%): R_{f} 0.78(8: 1$ petrol/ethyl acetate); $\nu_{\max } / \mathrm{cm}^{-1}$ (thin film) 2930; ${ }^{1} \mathrm{H}$ NMR (400 $\left.\mathrm{MHz} \mathrm{CDCl}_{3}\right) \delta 7.26(\mathrm{~d}, J=8.8 \mathrm{~Hz}, 2 \mathrm{H}, \mathrm{ArH}), 6.89(\mathrm{~d}, J=8.8 \mathrm{~Hz}$, $2 \mathrm{H}, \mathrm{ArH}), 6.27\left(\mathrm{dt}, J=15.7,7.0 \mathrm{~Hz}, 1 \mathrm{H}, \mathrm{CH}_{2} \mathrm{CH}=\mathrm{CH}\right), 5.58(\mathrm{dq}, J$ $\left.=15.7,2.0 \mathrm{~Hz}, 1 \mathrm{H}, \mathrm{CH}_{2} \mathrm{CH}=\mathrm{CH}\right), 4.49(\mathrm{~d}, J=11.4 \mathrm{~Hz}, 1 \mathrm{H}$, $\left.\mathrm{CHH}^{\prime} \mathrm{Ar}\right), 4.41\left(\mathrm{~d}, J=11.4 \mathrm{~Hz}, 1 \mathrm{H}, \mathrm{CHH}^{\prime} \mathrm{Ar}\right), 4.21(\mathrm{q}, J=6.8 \mathrm{~Hz}$, $1 \mathrm{H}, \mathrm{CHORCHOR}), 3.92-4.01(\mathrm{~m}, 3 \mathrm{H}, \mathrm{CHORCHCl}, \mathrm{CHORCH}-$ OR), $3.88(\mathrm{td}, J=7.3,4.0 \mathrm{~Hz}, 1 \mathrm{H}, \mathrm{EtCHOR}), 3.81(\mathrm{~s}, 3 \mathrm{H}, \mathrm{OMe})$, $3.78-3.82(\mathrm{~m}, 1 \mathrm{H}, \mathrm{CHOPMB}), 2.83(\mathrm{~d}, J=2.0 \mathrm{~Hz}, 1 \mathrm{H}, \mathrm{CCH}), 2.47$ (dddd, $\left.J=13.4,6.8,4.8 \mathrm{~Hz}, 1.5,1 \mathrm{H}, \mathrm{CHH} \mathrm{H}^{\prime} \mathrm{CHCl}\right), 2.33-2.40(\mathrm{~m}$, $\left.2 \mathrm{H}, \mathrm{CH}_{2} \mathrm{CH}=\mathrm{CH}\right), 2.19-2.30\left(\mathrm{~m}, 2 \mathrm{H}, 2 \times \mathrm{CHH}^{\prime} \mathrm{CHX}\right), 1.90$ (ddd, $\left.J=13.2,6.6,5.0 \mathrm{~Hz}, 1 \mathrm{H}, \mathrm{CHH} \mathrm{H}^{\prime} \mathrm{CHOPMB}\right), 1.48(\mathrm{qn}, J=7.3 \mathrm{~Hz}, 2 \mathrm{H}$, $\left.\mathrm{CH}_{3} \mathrm{CH}_{2}\right), 0.93\left(\mathrm{t}, J=7.3 \mathrm{~Hz}, 3 \mathrm{H}, \mathrm{CH}_{3}\right) ;{ }^{13} \mathrm{C}\left\{{ }^{1} \mathrm{H}\right\} \mathrm{NMR}(100 \mathrm{MHz}$, $\left.\mathrm{CDCl}_{3}\right) \delta 159.2(\mathrm{Ar}), 141.0\left(\mathrm{CH}_{2} \mathrm{CH}=\mathrm{CH}\right), 130.1(\mathrm{Ar}), 129.3(\mathrm{Ar})$, $113.8(\mathrm{Ar}), 111.6\left(\mathrm{CH}_{2} \mathrm{CH}=\mathrm{CH}\right), 85.5(\mathrm{CHORCHCl}), 84.9$ (EtCHOR), 82.2 (CHOPMB), 82.0 (CCH), 80.2 (CHORCHOR), 79.1 (CHORCHOR), 77.2 (CCH), 71.1 ( $\left.\mathrm{CH}_{2} \mathrm{Ar}\right), 58.9(\mathrm{CHCl}), 55.3$ $(\mathrm{OMe}), \quad 37.9\left(\mathrm{CH}_{2} \mathrm{CHCl}\right), 36.7\left(\mathrm{CH}_{2} \mathrm{CH}=\mathrm{CH}\right), 34.5$ ( $\left.\mathrm{CH}_{2} \mathrm{CHOPMB}\right), 26.6\left(\mathrm{CH}_{3} \mathrm{CH}_{2}\right), 10.2\left(\mathrm{CH}_{3}\right)$; MS (ESI-TOF) $\mathrm{m} /$ $z 427\left[{ }^{35} \mathrm{M}+\mathrm{Na}\right]^{+}, 429\left[{ }^{35} \mathrm{M}+\mathrm{Na}\right]{ }^{+}$; HRMS (ESI-TOF) $\mathrm{m} / z[\mathrm{M}+$ $\mathrm{Na}]^{+}$calcd for $\mathrm{C}_{23} \mathrm{H}_{29} \mathrm{O}_{4}{ }^{35} \mathrm{ClNa} 427.1647$; found 427.1634; $[\alpha]_{\mathrm{D}}^{20}$ $+34.2\left(c=1.0\right.$ in $\left.\mathrm{CHCl}_{3}\right)$.

$\left(2 S, 2^{\prime} R, 4 S, 4^{\prime} S, 5 R, 5^{\prime} R\right)-4^{\prime}$-Chloro-5-ethyl-5'-((E)-pent-2-en-4-yn1-yl)octahydro-[2,2'-bifuran]-4-ol (2c). To a stirred solution of ether $31(21 \mathrm{mg}, 50 \mu \mathrm{mol})$ in DCM $(5 \mathrm{~mL})$ was added $\mathrm{BCl}_{3}$.DMS $(120 \mu \mathrm{L}$, $2 \mathrm{M}$ in DCM, $0.24 \mathrm{mmol}$ ). The reaction was stirred for $5 \mathrm{~min}$ and then quenched with saturated aqueous $\mathrm{NaHCO}_{3}(5 \mathrm{~mL})$. The mixture was then extracted with DCM $(3 \times 10 \mathrm{~mL})$. The combined organic layers were dried $\left(\mathrm{Na}_{2} \mathrm{SO}_{4}\right)$, filtered, and concentrated in vacuo. Purification by flash column chromatography (3:1) petrol/ ethyl acetate) gave the title compound as a colorless oil (12 mg, 42 $\mu \mathrm{mol}, 84 \%) ; R_{f} 0.60$ (3:1 petrol/ethyl acetate); $\nu_{\max } / \mathrm{cm}^{-1}$ (thin film) 3420br, 3294m (CH), 2964m; ${ }^{1} \mathrm{H}$ NMR (400 MHz $\left.\mathrm{CDCl}_{3}\right) \delta 6.22$ $\left(\mathrm{dt}, J=15.4,7.4 \mathrm{~Hz}, 1 \mathrm{H}, \mathrm{CH}_{2} \mathrm{CH}=\mathrm{CH}\right), 5.59(\mathrm{dq}, J=15.4,2.0 \mathrm{~Hz}$, $\left.1 \mathrm{H}, \mathrm{CH}_{2} \mathrm{CH}=\mathrm{CH}\right), 4.39$ (ddd, $J=9.1,6.8,4.0 \mathrm{~Hz}, 1 \mathrm{H}$, CHORCHOR), 4.16 (dt, J = 9.1, $3.5 \mathrm{~Hz}, 1 \mathrm{H}, \mathrm{CHORCHOR}), 4.07$ $(\mathrm{dt}, J=6.8,5.4, \mathrm{~Hz}, 1 \mathrm{H}, \mathrm{CHClCHOR}), 4.03(\mathrm{dt}, J=6.1,2.0 \mathrm{~Hz}, 1 \mathrm{H}$, $\mathrm{CHCl}$ ), 3.97 (ddd, $J=7.8,5.4,4.3 \mathrm{~Hz}, 1 \mathrm{H}, \mathrm{CHOH}), 3.86(\mathrm{td}, J=6.9$, $1.8 \mathrm{~Hz}, 1 \mathrm{H}, \mathrm{EtCHOR}), 3.13(\mathrm{br}, 1 \mathrm{H}, \mathrm{OH}), 2.84(\mathrm{~d}, J=2.0 \mathrm{~Hz}, 1 \mathrm{H}$, $\mathrm{CCH}$ ), 2.51 (dddd, $J=14.4,7.4,5.3,2.0 \mathrm{~Hz}, 1 \mathrm{H}, \mathrm{CH} \mathrm{H}^{\prime} \mathrm{CH}=\mathrm{CH}$ ), 2.41 (dddd $J=14.4,7.4,5.3,2.0 \mathrm{~Hz}, 1 \mathrm{H}, \mathrm{CH} H^{\prime} \mathrm{CH}=\mathrm{CH}$ ), 2.30 (ddd, $\left.J=13.9,9.1,6.1 \mathrm{~Hz}, 1 \mathrm{H}, \mathrm{CH} \mathrm{H}^{\prime} \mathrm{CHOH}\right), 2.19$ (ddd, $J=13.9$, 6.8, $4.0 \mathrm{~Hz}, 1 \mathrm{H}, \mathrm{CHH}^{\prime} \mathrm{CHCl}$ ), 2.08 (ddd, $J=13.9,8.9,7.8 \mathrm{~Hz}, 1 \mathrm{H}$, $\mathrm{CHH}{ }^{\prime} \mathrm{CHCl}$ ), 1.76 (ddd, $\left.J=13.9,4.2,2.0 \mathrm{~Hz}, \mathrm{CH} H^{\prime} \mathrm{CHOH}\right), 1.39$ $\left(2 \mathrm{H}, \mathrm{qn}, J=7.6 \mathrm{~Hz}, \mathrm{CH}_{3} \mathrm{CH}_{2}\right), 0.95\left(3 \mathrm{H}, \mathrm{t}, J=7.6 \mathrm{~Hz}, 1 \mathrm{H}, \mathrm{CH}_{3}\right)$; ${ }^{13} \mathrm{C}\left\{{ }^{1} \mathrm{H}\right\}$ NMR (100 MHz, $\left.\mathrm{CDCl}_{3}\right) \delta 140.1\left(\mathrm{CH}_{2} \mathrm{CH}=\mathrm{CH}\right), 112.3$ $\left(\mathrm{CH}_{2} \mathrm{CH}=\mathrm{CH}\right), 88.9(\mathrm{EtCHOR}), 86.0(\mathrm{CHORCHCl}), 81.7(\mathrm{CCH})$, 79.8 (CHORCHOR), 78.2 (CHORCHOR), 77.2 (CCH), 74.8 $(\mathrm{CHOH}), 58.3(\mathrm{CHCl}), 38.2\left(\mathrm{CH}_{2} \mathrm{CHCl}\right), 36.6\left(\mathrm{CH}_{2} \mathrm{CH}=\mathrm{CH}\right)$, $34.3\left(\mathrm{CH}_{2} \mathrm{CHOH}\right), 26.0\left(\mathrm{CH}_{3} \mathrm{CH}_{2}\right), 10.2\left(\mathrm{CH}_{3}\right)$; MS (ESI-TOF) $\mathrm{m} /$ $z 307\left[{ }^{35} \mathrm{M}+\mathrm{Na}\right]^{+}, 309\left[{ }^{35} \mathrm{M}+\mathrm{Na}\right]^{+}$; HRMS (ESI-TOF) $\mathrm{m} / z[\mathrm{M}+$ $\mathrm{Na}]^{+}$calcd for $\mathrm{C}_{15} \mathrm{H}_{21} \mathrm{O}_{3}{ }^{35} \mathrm{ClNa} 307.1071$; found 307.1070; $[\alpha]_{\mathrm{D}}^{20}$ $+53.4\left(c=1.0\right.$ in $\left.\mathrm{CHCl}_{3}\right)$.

$\left(2 S, 2^{\prime} R, 4 R, 4^{\prime} S, 5 R, 5^{\prime} R\right)-4^{\prime}$-Chloro-5-ethyl-5' -((E)-pent-2-en-4-yn1-yl)octahydro-[2,2'-bifuran]-4-ol (2a). Alcohol 2c (2 mg, $7 \mu \mathrm{mol})$ and $\mathrm{PPh}_{3}(20.2 \mathrm{mg}, 77 \mu \mathrm{mol})$ were dissolved in THF $(0.3 \mathrm{~mL})$ and cooled to $0{ }^{\circ} \mathrm{C}$. DIAD $(15.6 \mu \mathrm{L}, 77 \mu \mathrm{mol})$, was added dropwise and 
the reaction stirred for $30 \mathrm{~min}$ before the addition of 4-nitrobenzoic acid (13 mg, $77 \mu \mathrm{mol}$ ). The reaction was then warmed to $\mathrm{rt}$ and stirred for $2 \mathrm{~h}$ before being concentrated in vacuo. Purification by short silica plug (DCM) gave the intermediate ester mixed with $\mathrm{DIADH}_{2}$. The mixture was then dissolved in $\mathrm{MeOH}(0.2 \mathrm{~mL})$, and cooled to $0{ }^{\circ} \mathrm{C}$ and $\mathrm{K}_{2} \mathrm{CO}_{3}(5.5 \mathrm{mg}, 40 \mu \mathrm{mol})$ was added. The reaction was stirred for $30 \mathrm{~min}$, then diluted with $\mathrm{H}_{2} \mathrm{O}(5 \mathrm{~mL})$, and extracted with EtOAc $(3 \times 10 \mathrm{~mL})$. The combined organic layers were then dried $\left(\mathrm{MgSO}_{4}\right)$, filtered, and concentrated in vacuo. Purification by flash column chromatography gave the title compound as a colorless oil $(0.5 \mathrm{mg}, 2 \mu \mathrm{mol}, 25 \%): R_{f} 0.62$ (3:1 petrol/ethyl acetate); $\nu_{\max } / \mathrm{cm}^{-1}$ (thin film) $3455 \mathrm{~m}, 3291 \mathrm{~m}, 2969 \mathrm{~m} ;{ }^{1} \mathrm{H}$ NMR $\left(500 \mathrm{MHz} \mathrm{CDCl}_{3}\right) \delta 6.25\left(\mathrm{dt}, J=15.8,7.3 \mathrm{~Hz}, 1 \mathrm{H}, \mathrm{CH}_{2} \mathrm{CH}=\mathrm{CH}\right)$, $5.58\left(\mathrm{dq}, J=15.8,2.2 \mathrm{~Hz}, 1 \mathrm{H}, \mathrm{CH}_{2} \mathrm{CH}=\mathrm{CH}\right), 4.30(\mathrm{br} \mathrm{m}, 1 \mathrm{H}$, $\mathrm{CHOH}), 4.15-4.23(\mathrm{~m}, 2 \mathrm{H}, \mathrm{CHORCHOR}), 3.98-4.03(\mathrm{~m}, 2 \mathrm{H}$, CHORCHCl), 3.73 (td, $J=7.0,2.8 \mathrm{~Hz}, 1 \mathrm{H}, \mathrm{EtCHOR}), 2.83$ (d, $J=$ $2.2 \mathrm{~Hz}, 1 \mathrm{H}, \mathrm{CCH}$ ), 2.47 (dddd, $J=12.3,7.3,5.0,2.2 \mathrm{~Hz}, 1 \mathrm{H}$, $\mathrm{CHH}^{\prime} \mathrm{CH}=\mathrm{CH}$ ), 2.37 (dddd, $J=12.3,7.3,6.3,2.2 \mathrm{~Hz}, 1 \mathrm{H}$, $\left.\mathrm{CH} H^{\prime} \mathrm{CH}=\mathrm{CH}\right), 2.28-2.17\left(\mathrm{~m}, 2 \mathrm{H}, \mathrm{CH}_{2} \mathrm{CHCl}\right), 2.10(\mathrm{dd}, J=13.6$, $6.3 \mathrm{~Hz}, 1 \mathrm{H}, \mathrm{CH} \mathrm{H}^{\prime} \mathrm{CHOH}$ ), 1.94 (ddd, $J=13.6,9.1,4.6 \mathrm{~Hz}, 1 \mathrm{H}$, $\left.\mathrm{CHH} H^{\prime} \mathrm{CHOH}\right), 1.70\left(\mathrm{dq}, J=13.6,7.6 \mathrm{~Hz}, 1 \mathrm{H}, \mathrm{CH}_{3} \mathrm{CHH}^{\prime}\right), 1.61(\mathrm{dq}$, $\left.J=13.6,7.6 \mathrm{~Hz}, 1 \mathrm{H}, \mathrm{CH}_{3} \mathrm{CHH}^{\prime}\right), 0.99\left(\mathrm{t}, J=7.6 \mathrm{~Hz}, 3 \mathrm{H}, \mathrm{CH}_{3}\right)$; ${ }^{13} \mathrm{C}\left\{{ }^{1} \mathrm{H}\right\}$ NMR $\left(125 \mathrm{MHz}, \mathrm{CDCl}_{3}\right) \delta 140.8\left(\mathrm{CH}_{2} \mathrm{CH}=\mathrm{CH}\right), 117.8$ $\left(\mathrm{CH}_{2} \mathrm{CH}=\mathrm{CH}\right), 85.7(\mathrm{CHORCHCl}), 84.7$ (EtCHOR), $81.9(\mathrm{CCH})$, 80.3 (CHORCHOR), 78.3 (CHORCHOR), 77.2 (CCH), 72.6 $(\mathrm{CHOH}), 58.8(\mathrm{CHCl}), 37.9\left(\mathrm{CH}_{2} \mathrm{CHOH}\right), 37.9\left(\mathrm{CH}_{2} \mathrm{CHCl}\right), 36.7$ $\left(\mathrm{CH}_{2} \mathrm{CH}=\mathrm{CH}\right), 22.0\left(\mathrm{CH}_{3} \mathrm{CH}_{2}\right), 10.5\left(\mathrm{CH}_{3}\right) ; \mathrm{MS}$ (ESI-TOF) $\mathrm{m} / z$ $307\left[{ }^{35} \mathrm{M}+\mathrm{Na}\right]^{+}, 309\left[{ }^{35} \mathrm{M}+\mathrm{Na}\right]^{+}$; HRMS (ESI-TOF) $\mathrm{m} / z[\mathrm{M}+$ $\mathrm{Na}]^{+}$calcd for $\mathrm{C}_{15} \mathrm{H}_{21} \mathrm{O}_{3}{ }^{35} \mathrm{ClNa} 307.1071$; found 307.1070; $[\alpha]_{\mathrm{D}}^{20}$ $+66.0\left(c=0.05\right.$ in $\left.\mathrm{CHCl}_{3}\right)$.

$\left(2 S, 2^{\prime} R, 4 S, 4^{\prime} S, 5 S, 5^{\prime} R\right)-5-A l l y l-5^{\prime}$-ethyl-4'-((4-methoxybenzyl)oxy)octahydro-[2,2'-bifuran]-4-ol (34). To a solution of $\left(2 S, 2^{\prime} R, 4 S, 4^{\prime} S, 5 S, 5^{\prime} R\right)$-5-allyl-4-((4-bromobenzyl)oxy)-5' -ethyl-4' ((4-methoxybenzyl)oxy)octahydro-2,2'-bifuran 32 (50 mg, $94 \mu \mathrm{L})$ and bis(methoxyethyl)amine $(44 \mu \mathrm{L}, 39 \mathrm{mg}, 0.3 \mathrm{mmol})$ in THF (4 $\mathrm{mL}$ ) at $-78{ }^{\circ} \mathrm{C}$ was titrated a solution of $\operatorname{LiDBB}$ (ca. $1 \mathrm{~mL}$ of a solution prepared by sonicating DBB $(0.75 \mathrm{~g}, 2.8 \mathrm{mmol})$, lithium (20 $\mathrm{mg}, 2.8 \mathrm{mmol})$ in THF $(3 \mathrm{~mL})$ for $2 \mathrm{~h}$ ). The reaction progress was monitored by TLC. When no more starting material was detected, the reaction was quenched by the addition of saturated aqueous $\mathrm{NH}_{4} \mathrm{Cl}$ and diluted with EtOAc and then allowed to warm to rt. The aqueous layer was separated and extracted with EtOAc. The combined organic layers were dried $\left(\mathrm{MgSO}_{4}\right)$, filtered, and concentrated in vacuo. Purification by flash column chromatography (5:1 petrol/ethyl acetate) gave the title compound as a colorless oil $(23 \mathrm{mg}, 62$ $\mu \mathrm{mol}, 66 \%)$ and benzyl-protected intermediate $33(8 \mathrm{mg}, 17 \mu \mathrm{mol}$, $18 \%$ ). Data for 34: $\nu_{\max } / \mathrm{cm}^{-1}$ (thin film) $3437 \mathrm{br}, 2960 \mathrm{~m}, 2934 \mathrm{~m} ; R_{f}$ 0.38 (5:1 petrol/ethyl acetate); ${ }^{1} \mathrm{H}$ NMR $\left(500 \mathrm{MHz} \mathrm{C}_{6} \mathrm{D}_{6}\right) \delta 7.15$ (d, $J=8.6 \mathrm{~Hz}, 2 \mathrm{H}, \operatorname{ArH}), 6.81(\mathrm{~d}, J=8.6 \mathrm{~Hz}, 2 \mathrm{H}, \operatorname{Ar} H), 6.07(\mathrm{ddt}, J=$ 17.0, 10.0, 7.0 Hz, $\left.1 \mathrm{H}, \mathrm{CH}=\mathrm{CH}_{2}\right), 5.20(\mathrm{dq}, J=17.0,2.0 \mathrm{~Hz}, 1 \mathrm{H}$, $\left.\mathrm{CH}=\mathrm{CHH}^{\prime}\right), 5.08\left(\mathrm{ddt}, J=10.0,2.0 \mathrm{~Hz}, 1 \mathrm{H}, \mathrm{CH}=\mathrm{CHH}^{\prime}\right), 4.46$ (ddd, $J=10.8,5.7,2.0 \mathrm{~Hz}, 1 \mathrm{H}, \mathrm{CHORCHOR}), 4.19$ (d, $J=11.6 \mathrm{~Hz}$, $\left.1 \mathrm{H}, \mathrm{CHH}^{\prime} \mathrm{Ar}\right), 4.14\left(\mathrm{~d}, J=11.6 \mathrm{~Hz}, 1 \mathrm{H}, \mathrm{CHH}^{\prime} \mathrm{Ar}\right), 3.89-3.93(\mathrm{~m}$, $3 \mathrm{H}$, EtCHOR, CHORCHOR, CHOH), $3.76(\mathrm{~d}, J=10.7 \mathrm{~Hz}, 1 \mathrm{H}$, $\mathrm{OH}), 3.55$ (td, $J=7.0,2.8 \mathrm{~Hz}, 1 \mathrm{H}, \mathrm{CHORCHOH}), 3.40-3.44(\mathrm{~m}$, $1 \mathrm{H}, \mathrm{CHOPMB}), 3.31(\mathrm{~s}, 3 \mathrm{H}, \mathrm{OMe}), 2.67-2.78\left(\mathrm{~m}, 2 \mathrm{H}, \mathrm{CH}_{2} \mathrm{CH}=\right.$ $\mathrm{CH}_{2}$ ), 1.78 (ddd, $J=13.6,10.2,5.3 \mathrm{~Hz}, 1 \mathrm{H}, \mathrm{CHH}^{\prime} \mathrm{CHOH}$ ), 1.70 (dd, $\left.J=13.6,2.8 \mathrm{~Hz}, 1 \mathrm{H}, \mathrm{CH} H^{\prime} \mathrm{CHOH}\right), 1.65(\mathrm{ddd}, J=13.2,5.5,2.0 \mathrm{~Hz}$, $\left.1 \mathrm{H}, \mathrm{CHH}^{\prime} \mathrm{CHOPMB}\right), 1.44\left(\mathrm{dq}, J=14.6,7.3 \mathrm{~Hz}, 1 \mathrm{H}, \mathrm{CH}_{3} \mathrm{CHH}^{\prime}\right)$, $1.26-1.33\left(\mathrm{~m}, 1 \mathrm{H}, \mathrm{CH}_{3} \mathrm{CHH}^{\prime}\right), 1.12(\mathrm{ddd} J=13.2,10.3,6.5 \mathrm{~Hz}, 1 \mathrm{H}$, CHH $H^{\prime}$ CHOMPB $), 0.83\left(\mathrm{t}, J=7.4 \mathrm{~Hz}, 3 \mathrm{H}, \mathrm{CH}_{3}\right) ;{ }^{13} \mathrm{C}\{1 \mathrm{H}\} \mathrm{NMR}$ $\left(125 \mathrm{MHz} \mathrm{C}_{6} \mathrm{D}_{6}\right) \delta 159.8(\mathrm{Ar}), 136.0\left(\mathrm{CH}=\mathrm{CH}_{2}\right), 130.7(\mathrm{Ar}), 129.3$ (Ar), $127.5(\mathrm{Ar}), 116.6\left(\mathrm{CH}=\mathrm{CH}_{2}\right) 114.1(\mathrm{Ar}), 86.3(\mathrm{EtCHOR})$, 84.0 (CHORCHCl), 82.2 (CHOPMB), 79.9 (CHORCHOR), 78.9 (CHORCHOR), $71.4(\mathrm{CHOH}), 70.8(\mathrm{CHOH}), 54.8(\mathrm{OMe}), 34.9$ $\left(\mathrm{CH}_{2} \mathrm{CHOH}\right), 34.8\left(\mathrm{CH}_{2} \mathrm{CHOPMB}\right), 34.5\left(\mathrm{CH}_{2} \mathrm{CH}=\mathrm{CH}_{2}\right), 27.4$ $\left(\mathrm{CH}_{3} \mathrm{CH}_{2}\right), 10.4\left(\mathrm{CH}_{3}\right)$; MS (ESI-TOF) $\mathrm{m} / z 385[\mathrm{M}+\mathrm{Na}]^{+}$; HRMS (ESI-TOF) $\mathrm{m} / z[\mathrm{M}+\mathrm{Na}]^{+}$calcd for $\mathrm{C}_{21} \mathrm{H}_{30} \mathrm{O}_{5} \mathrm{Na} 385.1985$; found 385.1985; $[\alpha]_{\mathrm{D}}^{20}+38.1\left(c=1.0\right.$ in $\left.\mathrm{CHCl}_{3}\right)$. $\left(2 S, 2^{\prime} R, 4 R, 4^{\prime} S, 5 S, 5^{\prime} R\right)-5$-Allyl-4-chloro-5' - ethyl-4' - ((4methoxybenzyl)oxy)octahydro-2,2'-bifuran (35). Alcohol 34 (24 $\mathrm{mg}, 66 \mu \mathrm{mol})$ was dissolved in $\mathrm{DCM} / \mathrm{CCl}_{4}(2.4 \mathrm{~mL} / 0.6 \mathrm{~mL})$ and $\mathrm{PPh}_{3}(72.5 \mathrm{mg}, 396 \mu \mathrm{mol})$ was added and the reaction stirred for 2.5 h. Direct purification of the reaction mixture via flash column chromatography (DCM $\rightarrow$ 5:1 petrol/ethyl) gave the title compound as a colorless oil $(24 \mathrm{mg}, 64 \mu \mathrm{mol}, 96 \%): R_{f} 0.89$ (6:1 petrol/ethyl acetate); $\nu_{\max } / \mathrm{cm}^{-1}$ (thin film) $2916 \mathrm{~m}, 2930 \mathrm{~m}, 2876 \mathrm{~m} ;{ }^{1} \mathrm{H}$ NMR $\left(500 \mathrm{MHz} \mathrm{C}_{6} \mathrm{D}_{6}\right) 7.19$ (d, $\left.J=8.5 \mathrm{~Hz}, 2 \mathrm{H}, \operatorname{ArH}\right), 6.81(\mathrm{~d}, J=8.5 \mathrm{~Hz}$, $2 \mathrm{H}, \mathrm{ArH}), 5.78\left(\mathrm{ddt}, J=16.5,10.0,7.0 \mathrm{~Hz}, 1 \mathrm{H}, \mathrm{CH}=\mathrm{CH}_{2}\right), 4.96-$ $5.01\left(\mathrm{~m}, 1 \mathrm{H}, \mathrm{CH}=\mathrm{CH}_{2}\right), 4.27\left(\mathrm{~d}, J=11.5 \mathrm{~Hz}, 1 \mathrm{H}, \mathrm{CHH}^{\prime} \mathrm{Ar}\right), 4.21$ (d, $\left.J=11.5 \mathrm{~Hz}, 1 \mathrm{H}, \mathrm{CH} H^{\prime} \mathrm{Ar}\right), 4.00-4.13(\mathrm{~m}, 3 \mathrm{H}, \mathrm{CHORCHOR}$, CHClCHOR), 3.96 (ddd, $J=7.0,6.0,3.0 \mathrm{~Hz}, 1 \mathrm{H}, \mathrm{EtCHOR}$ ), 3.77 $(\mathrm{dt}, J=7.0,5.0 \mathrm{~Hz}, 1 \mathrm{H}, \mathrm{CHCl}), 3.55(\mathrm{ddd}, J=6.5,3.0,2.5 \mathrm{~Hz}, 1 \mathrm{H}$, CHOPMB), 3.30 (s, 3H, OMe), 2.06-2.17 (m, 4H, $\mathrm{CH}_{2} \mathrm{CH}=\mathrm{CH}_{2}$, $\mathrm{CH}_{2} \mathrm{CHCl}$ ), 2.04 (ddd, $J=13.4,6.0,2.5 \mathrm{~Hz}, 1 \mathrm{H}, \mathrm{CHH}^{\prime} \mathrm{CHOPMB}$ ), 1.57 (ddd, $J=13.4,9.0,6.5 \mathrm{~Hz}, 1 \mathrm{H}, \mathrm{CH} H^{\prime} \mathrm{CHOPMB}$ ), $1.32-1.48$ $\left(\mathrm{m}, 2 \mathrm{H}, \mathrm{CH}_{3} \mathrm{CH}_{2}\right), 0.90\left(\mathrm{t}, J=7.5 \mathrm{~Hz}, 3 \mathrm{H}, \mathrm{CH}_{3} \mathrm{CH}_{2}\right) ;{ }^{13} \mathrm{C}\left\{{ }^{1} \mathrm{H}\right\} \mathrm{NMR}$ $\left(125 \mathrm{MHz} \mathrm{C}_{6} \mathrm{D}_{6}\right) \delta 159.8(\mathrm{Ar}), 134.1\left(\mathrm{CH}=\mathrm{CH}_{2}\right), 130.9(\mathrm{Ar}), 129.4$ (Ar), 127.5 (Ar), $117.4\left(\mathrm{CH}=\mathrm{CH}_{2}\right), 114.1$ (Ar), 86.7 (CHORCHCl), 85.9 (EtCHOR), 82.8 (CHOPMB), 80.8 (CHORCHOR), 80.4 (CHORCHOR), $70.9\left(\mathrm{CH}_{2} \mathrm{Ar}\right), 59.8$ $(\mathrm{CHCl}), 54.8(\mathrm{OMe}), 38.9\left(\mathrm{CH}_{2} \mathrm{CHCl}\right), 38.3\left(\mathrm{CH}_{2} \mathrm{CH}=\mathrm{CH}_{2}\right)$, $35.7\left(\mathrm{CH}_{2} \mathrm{CHOPMB}\right), 27.8\left(\mathrm{CH}_{3} \mathrm{CH}_{2}\right), 10.5\left(\mathrm{CH}_{3}\right)$; MS (ESI-TOF) $\mathrm{m} / z 403\left[{ }^{35} \mathrm{M}+\mathrm{Na}\right]^{+}, 405\left[{ }^{37} \mathrm{M}+\mathrm{Na}\right]^{+}$; HRMS (ESI-TOF) $\mathrm{m} / z[\mathrm{M}$ $+\mathrm{Na}]^{+}$calcd for $\mathrm{C}_{21} \mathrm{H}_{29} \mathrm{O}_{4}{ }^{35} \mathrm{ClNa}$ 403.1647; found 403.1638; $[\alpha]_{\mathrm{D}}^{20}$ $+8.1\left(c=1.0\right.$ in $\left.\mathrm{CHCl}_{3}\right)$.

$\left(2 R, 2^{\prime} S, 4 S, 4^{\prime} R, 5 R, 5^{\prime} S\right)-5^{\prime}$-Allyl-4' -chloro-5-ethyloctahydro-[2,2'bifuran]-4-ol (36). Chloride $35(24 \mathrm{mg}, 66 \mu \mathrm{mol})$ was dissolved in $\operatorname{DCM}(6 \mathrm{~mL})$ and $\mathrm{BCl}_{3} \cdot \mathrm{DMS}(165 \mu \mathrm{L}, 2 \mathrm{M}$ in DCM, $0.33 \mathrm{mmol})$ was added dropwise. The reaction was stirred for $5 \mathrm{~min}$ and then quenched by the addition of saturated aqueous $\mathrm{NaHCO}_{3}(4 \mathrm{~mL})$. The mixture was then extracted with DCM $(3 \times 10 \mathrm{~mL})$. The combined organic layers were then dried $\left(\mathrm{Na}_{2} \mathrm{SO}_{4}\right)$, filtered, and concentrated in vacuo. Purification via flash column chromatography (4:1 petrol/ethyl acetate) gave the title compound as a colorless oil (16 mg, $62 \mu \mathrm{mol}, 94 \%): R_{f} 0.34$ (5:1 petrol/ethyl acetate); $\nu_{\max } / \mathrm{cm}^{-1}$ (thin film) 3423br (OH), 2965m, 1437w, 1067s; ${ }^{1} \mathrm{H}$ NMR (400 MHz $\left.\mathrm{CDCl}_{3}\right) \delta 5.83\left(\mathrm{ddt}, J=17.0,9.9,7.0 \mathrm{~Hz}, 1 \mathrm{H}, \mathrm{CH}=\mathrm{CH}_{2}\right), 5.13(\mathrm{~d}, J$ $\left.=17.0 \mathrm{~Hz}, 1 \mathrm{H}, \mathrm{CH}=\mathrm{CHH}^{\prime}\right), 5.11\left(\mathrm{~d}, J=9.9 \mathrm{~Hz}, 1 \mathrm{H}, \mathrm{CH}=\mathrm{CHH}^{\prime}\right)$, 4.06-4.15 (m, 3H, CHOH, CHORCHOR), 4.00-4.06 (m, $2 \mathrm{H}$, CHClCHOR), $3.68(\mathrm{td}, J=6.6,2.8 \mathrm{~Hz}, 1 \mathrm{H}, \mathrm{EtCHOR}), 2.30-2.40$ $\left(\mathrm{m}, 2 \mathrm{H}, \mathrm{CH}_{2} \mathrm{CH}=\mathrm{CH}_{2}\right), 2.17-2.29\left(\mathrm{~m}, 2 \mathrm{H}, \mathrm{CH}_{2} \mathrm{CHCl}\right), 1.85-1.98$ $\left(\mathrm{m}, 2 \mathrm{H}, \mathrm{CH}_{2} \mathrm{CHOH}\right), 1.74($ br s, $1 \mathrm{H}, \mathrm{OH}), 1.44-1.57(\mathrm{~m}, 2 \mathrm{H}$, $\left.\mathrm{CH}_{3} \mathrm{CH}_{2}\right), 0.96\left(\mathrm{t}, J=7.4 \mathrm{~Hz}, 3 \mathrm{H}, \mathrm{CH}_{3}\right) ;{ }^{13} \mathrm{C}\left\{{ }^{1} \mathrm{H}\right\} \mathrm{NMR}(100 \mathrm{MHz}$, $\left.\mathrm{CDCl}_{3}\right) \delta 133.5\left(\mathrm{CH}=\mathrm{CH}_{2}\right), 117.9\left(\mathrm{CH}=\mathrm{CH}_{2}\right), 88.2(\mathrm{EtCHOR})$, 86.4 (CHORCHCl), 80.0 (CHORCHOR), 79.2 (CHORCHOR), $75.7(\mathrm{CHOH}), \quad 59.2(\mathrm{CHCl}), \quad 38.0\left(\mathrm{CH}_{2} \mathrm{CH}=\mathrm{CH}_{2}\right), \quad 38.0$ $\left(\mathrm{CH}_{2} \mathrm{CHCl}\right), 37.6\left(\mathrm{CH}_{2} \mathrm{CHOH}\right), 27.1\left(\mathrm{CH}_{3} \mathrm{CH}_{2}\right), 10.2\left(\mathrm{CH}_{3}\right) ; \mathrm{MS}$ (ESI-TOF) $m / z 283\left[{ }^{35} \mathrm{M}+\mathrm{Na}\right]^{+}, 285\left[{ }^{37} \mathrm{M}+\mathrm{Na}\right]^{+}$; HRMS (ESITOF) $m / z[\mathrm{M}+\mathrm{Na}]^{+}$calcd for $\mathrm{C}_{13} \mathrm{H}_{21} \mathrm{O}_{3}{ }^{35} \mathrm{ClNa} 283.1071$; found 283.1073; $[\alpha]_{\mathrm{D}}^{20}-38.6\left(c=1.0\right.$ in $\left.\mathrm{CHCl}_{3}\right)$.

$\left(2 R, 2^{\prime} S, 4 R, 4^{\prime} R, 5 R, 5^{\prime} S\right)-5^{\prime}-$ Allyl-4' -chloro-5-ethyloctahydro-[2, $2^{\prime}$ bifuran]-4-yl 4-nitrobenzoate. Alcohol $36(15.5 \mathrm{mg}, 60 \mu \mathrm{mol})$ and $\mathrm{PPh}_{3}(156 \mathrm{mg}, 600 \mu \mathrm{mol})$ were dissolved in THF $(0.8 \mathrm{~mL})$ and cooled to $0{ }^{\circ} \mathrm{C}$. DIAD $(120 \mu \mathrm{L}, 600 \mu \mathrm{mol})$ was added dropwise, and the reaction was stirred for $30 \mathrm{~min}$ before the addition of 4nitrobenzoic acid $(99.6 \mathrm{mg}, 600 \mu \mathrm{mol})$. The reaction was then warmed to $\mathrm{rt}$ and stirred for $2 \mathrm{~h}$ before being concentrated in vacuo. Purification via flash column chromatography (DCM) gave the title compound as a colorless oil $(20 \mathrm{mg}, 49 \mu \mathrm{mol}, 81 \%): R_{f} 0.43(8: 1$ petrol/acetone); $\nu_{\max } / \mathrm{cm}^{-1}$ (thin film) $2972 \mathrm{w}, 1724 \mathrm{~s}(\mathrm{CO}), 1528 \mathrm{~s}$, $1350 \mathrm{~m} ;{ }^{1} \mathrm{H}$ NMR $\left(400 \mathrm{MHz} \mathrm{CDCl}_{3}\right) \delta 8.31(\mathrm{~d}, J=9.1 \mathrm{~Hz}, 2 \mathrm{H}, \mathrm{ArH})$, $8.20(\mathrm{~d}, J=9.1 \mathrm{~Hz}, 2 \mathrm{H}, \operatorname{ArH}), 5.77(\mathrm{ddt}, J=17.0,10.0,7.1 \mathrm{~Hz}, 1 \mathrm{H}$, $\mathrm{CH}=\mathrm{CH}_{2}$ ), 5.54 (ddd, $\left.J=6.5,3.6,1.6 \mathrm{~Hz}, 1 \mathrm{H}, \mathrm{CHOPNB}\right), 5.07$ (dd, $\left.J=17.0,1.2 \mathrm{~Hz}, 1 \mathrm{H}, \mathrm{CH}=\mathrm{CHH}^{\prime}\right), 5.05(\mathrm{dd}, J=10.0,1.2 \mathrm{~Hz}, 1 \mathrm{H}$, $\left.\mathrm{CH}=\mathrm{CHH}^{\prime}\right), 4.24(\mathrm{dt}, J=7.5,6.1 \mathrm{~Hz}, 1 \mathrm{H}, \mathrm{CHORCHOR}), 4.01-$ $4.11(\mathrm{~m}, 2 \mathrm{H}$, CHClCHOR $), 3.97(\mathrm{dt}, J=8.3,6.0 \mathrm{~Hz}, 1 \mathrm{H}$, CHORCHOR), $3.81 \mathrm{dd} J=7.4,6.1,3.5 \mathrm{~Hz}, 1 \mathrm{H}$, EtCHOR), 2.57 (ddd, $\left.J=14.9,8.5,6.5 \mathrm{~Hz}, 1 \mathrm{H}, \mathrm{CH} \mathrm{H}^{\prime} \mathrm{CHOPNB}\right), 2.22-2.38(\mathrm{~m}, 4 \mathrm{H}$, 
$\mathrm{CH}_{2} \mathrm{CH}=\mathrm{CH}_{2}, \mathrm{CH}_{2} \mathrm{CHCl}$ ), $2.02(\mathrm{ddd}, J=14.8,6.0,1.7 \mathrm{~Hz}, 1 \mathrm{H}$, $\left.\mathrm{CH} H^{\prime} \mathrm{CHOPNB}\right), 1.63-1.85\left(\mathrm{~m}, 2 \mathrm{H}, \mathrm{CH}_{3} \mathrm{CH}_{2}\right), 0.99(\mathrm{t}, J=7.4 \mathrm{~Hz}$, $\left.3 \mathrm{H}, \mathrm{CH}_{3}\right) ;{ }^{13} \mathrm{C}\left\{{ }^{1} \mathrm{H}\right\}$ NMR (100 $\left.\mathrm{MHz}, \mathrm{CDCl}_{3}\right) \delta 164.0(\mathrm{C}=\mathrm{O})$, $150.7(\mathrm{Ar}), 135.4(\mathrm{Ar}), 133.3\left(\mathrm{CH}=\mathrm{CH}_{2}\right), 130.7(\mathrm{Ar}), 123.7(\mathrm{Ar})$, $118.0\left(\mathrm{CH}=\mathrm{CH}_{2}\right), 86.4(\mathrm{CHORCHCl}), 83.7$ (EtCHOR), 79.6 (CHORCHOR), 79.0 (CHORCHOR), 75.9 (CHOPNB), 59.1 $(\mathrm{CHCl}), 38.2\left(\mathrm{CH}_{2} \mathrm{CH}=\mathrm{CH}_{2}\right), 37.9\left(\mathrm{CH}_{2} \mathrm{CHCl}\right), 35.9$ ( $\left.\mathrm{CH}_{2} \mathrm{CHOPNB}\right), 22.2\left(\mathrm{CH}_{3} \mathrm{CH}_{2}\right), 10.6\left(\mathrm{CH}_{3}\right)$; MS (ESI-TOF) $\mathrm{m} /$ $z 432\left[{ }^{35} \mathrm{M}+\mathrm{Na}^{+}\right], 434\left[{ }^{37} \mathrm{M}+\mathrm{Na}^{+}\right]$; HRMS (ESI-TOF) $m / z[\mathrm{M}+$ $\mathrm{Na}]^{+}$calcd for $\mathrm{C}_{20} \mathrm{H}_{24} \mathrm{NO}_{6}{ }^{35} \mathrm{ClNa}$ 432.1184; found 432.1184; $[\alpha]_{\mathrm{D}}^{20}$ $-12.4\left(c=1.0\right.$ in $\left.\mathrm{CHCl}_{3}\right)$.

$\left(2 R, 2^{\prime} S, 4 R, 4^{\prime} R, 5 R, 5^{\prime} S\right)-5^{\prime}$-Allyl-4' -chloro-5-ethyloctahydro-[2,2'bifuran]-4-ol (37). ( $\left.2 R, 2^{\prime} S, 4 R, 4^{\prime} R, 5 R, 5^{\prime} S\right)-5^{\prime}$-allyl-4' -chloro-5-ethyloctahydro-[2,2'-bifuran]-4-yl 4-nitrobenzoate $(20 \mathrm{mg}, 49 \mu \mathrm{mol})$ was dissolved in $\mathrm{MeOH}(2 \mathrm{~mL})$ and cooled to $0{ }^{\circ} \mathrm{C}$, and $\mathrm{K}_{2} \mathrm{CO}_{3}(40 \mathrm{mg}$, $290 \mu \mathrm{mol})$ was added. The reaction was stirred for $30 \mathrm{~min}$ and then diluted with $\mathrm{H}_{2} \mathrm{O}(5 \mathrm{~mL})$ and extracted with EtOAc $(3 \times 10 \mathrm{~mL})$. The combined organic layers were then dried $\left(\mathrm{MgSO}_{4}\right)$, filtered, and concentrated in vacuo. Purification by flash column chromatography (4:1 petrol/ethyl acetate) gave the title compound as a colorless oil (13 mg $48 \mu \mathrm{mol}, 98 \%): R_{f} 0.32$ (5:1 petrol/ethyl acetate); $\nu_{\max } / \mathrm{cm}^{-1}$ (thin film) $3461 \mathrm{br}(\mathrm{OH}), 2968 \mathrm{~m}, 1430 \mathrm{~m}, 1050 \mathrm{~s} ;{ }^{1} \mathrm{H}$ NMR (400 $\left.\mathrm{MHz} \mathrm{CDCl}_{3}\right) \delta 5.82$ (ddt, $J=17.0,10.0,6.8 \mathrm{~Hz}, 1 \mathrm{H}, \mathrm{CH}=\mathrm{CH}_{2}$ ), $5.17\left(\mathrm{dd}, J=17.0,1.5 \mathrm{~Hz}, 1 \mathrm{H}, \mathrm{CH}=\mathrm{CHH}^{\prime}\right), 5.15(\mathrm{dd}, J=10.0,1.5$ $\left.\mathrm{Hz}, 1 \mathrm{H}, \mathrm{CH}=\mathrm{CHH}^{\prime}\right), 4.44(\mathrm{ddd}, J=9.1,6.8,2.6 \mathrm{~Hz}, 1 \mathrm{H}$, CHORCHOR), 4.16 (dt, $J=9.8,2.6 \mathrm{~Hz}, 1 \mathrm{H}, \mathrm{CHORCHOR}), 4.11-$ $4.14(\mathrm{~m}, 1 \mathrm{H}, \mathrm{CHORCHCl}), 3.99-4.04(\mathrm{~m}, 2 \mathrm{H}, \mathrm{CHOH}, \mathrm{CHCl}), 3.54$ (tdfz, $J=6.9,2.5 \mathrm{~Hz}, 1 \mathrm{H}$, EtCHOR), 3.24 (br s, $1 \mathrm{H}, \mathrm{OH}), 2.33-2.47$ $\left(\mathrm{m}, 2 \mathrm{H}, \mathrm{CH}_{2} \mathrm{CH}=\mathrm{CH}_{2}\right), 2.25(\mathrm{ddd}, J=14.0,9.8,5.5 \mathrm{~Hz}, 1 \mathrm{H}$, $\left.\mathrm{CH} \mathrm{H}^{\prime} \mathrm{CHOH}\right), 2.15$ (ddd, $J=13.8,6.8,3.7 \mathrm{~Hz}, 1 \mathrm{H}, \mathrm{CHH}^{\prime} \mathrm{CHCl}$ ), 2.00 (ddd, $J=13.8,9.6,7.8 \mathrm{~Hz}, 1 \mathrm{H}, \mathrm{CH} H^{\prime} \mathrm{CHCl}$ ), 1.81 (dd, $J=14.0$, $\left.3.3 \mathrm{~Hz}, 1 \mathrm{H}, \mathrm{CHH}^{\prime} \mathrm{CHOH}\right), 1.65-1.74\left(\mathrm{~m}, 2 \mathrm{H}, \mathrm{CH}_{3} \mathrm{CH}_{2}\right), 0.97(\mathrm{t}, J=$ $\left.7.6 \mathrm{~Hz}, 3 \mathrm{H}, \mathrm{CH}_{3}\right) ;{ }^{13} \mathrm{C}\left\{{ }^{1} \mathrm{H}\right\}$ NMR $\left(100 \mathrm{MHz}, \mathrm{CDCl}_{3}\right) \delta 132.7$ $\left(\mathrm{CH}=\mathrm{CH}_{2}\right), 118.6\left(\mathrm{CH}=\mathrm{CH}_{2}\right), 86.6(\mathrm{CHORCHCl}), 85.7(\mathrm{EtCH}-$ OR), 79.0 (CHORCHOR), 77.9 (CHORCHOR), 70.9 ( $\mathrm{CHOH})$, $58.4(\mathrm{CHCl}), 38.2\left(\mathrm{CH}_{2} \mathrm{CHCl}\right), 37.6\left(\mathrm{CH}_{2} \mathrm{CH}=\mathrm{CH}_{2}\right), 34.7$ $\left(\mathrm{CH}_{2} \mathrm{CHOH}\right), 21.8\left(\mathrm{CH}_{3} \mathrm{CH}_{2}\right), 10.5\left(\mathrm{CH}_{3}\right) ; \mathrm{MS}$ (ESI-TOF) $\mathrm{m} / \mathrm{z}$ $283\left[{ }^{35} \mathrm{M}+\mathrm{Na}^{+}\right], 285\left[{ }^{37} \mathrm{M}+\mathrm{Na}^{+}\right]$; HRMS (ESI-TOF) $m / z[\mathrm{M}+$ $\mathrm{Na}]^{+}$calcd for $\mathrm{C}_{13} \mathrm{H}_{21} \mathrm{O}_{3}{ }^{35} \mathrm{ClNa} 283.1071$; found 283.1072; $[\alpha]_{\mathrm{D}}^{20}$ $-40.2\left(c=1.0\right.$ in $\left.\mathrm{CHCl}_{3}\right)$.

$\left(2 R, 2^{\prime} S, 4 R, 4^{\prime} R, 5 R, 5^{\prime} S\right)-4^{\prime}$-Chloro-5-ethyl-5' -((E)-pent-2-en-4-yn1-yl)octahydro-[2,2'-bifuran]-4-ol, L. majuscula Enyne (2b). To a stirred solution of alkene $37(12 \mathrm{mg}, 46 \mu \mathrm{mol})(62 \mathrm{mg}, 0.16 \mathrm{mmol})$ in dry degassed DCM $(1.4 \mathrm{~mL})$ were added crotonaldehyde $(38 \mu \mathrm{L}$, $0.46 \mathrm{mmol}$ ) and Grubbs' second generation catalyst $(3.9 \mathrm{mg}, 4.6$ $\mu \mathrm{mol})$. The reaction mixture was stirred for $1.5 \mathrm{~h}$ at $40{ }^{\circ} \mathrm{C}$ and then cooled to rt and quenched with the addition of DMSO $(30 \mu \mathrm{L})$ and stirred for $16 \mathrm{~h}$. The mixture was concentrated in vacuo and purified by flash column chromatography (5:1 petrol ethyl acetate) to give the intermediate enal (38) (12.6 mg), which was used immediately in the next reaction: MS (ESI-TOF) $m / z 311\left[{ }^{35} \mathrm{M}+\mathrm{Na}^{+}\right], 313\left[{ }^{37} \mathrm{M}+\right.$ $\left.\mathrm{Na}^{+}\right]$; HRMS (ESI-TOF) $\mathrm{m} / z[\mathrm{M}+\mathrm{Na}]^{+}$calcd for $\mathrm{C}_{14} \mathrm{H}_{21} \mathrm{O}_{4}{ }^{35} \mathrm{ClNa}$ 311.1021; found 311.1026.

To a solution of (diazomethyl)trimethylsilane $(230 \mu \mathrm{L}, 2 \mathrm{M}$ in ether, $0.46 \mathrm{mmol})$ in THF $(1 \mathrm{~mL})$ at $-78{ }^{\circ} \mathrm{C}$ was added BuLi $(288$ $\mu \mathrm{L}, 1.6 \mathrm{M}$ in hexanes, $0.46 \mathrm{mmol}$ ) dropwise. The reaction mixture was stirred for $30 \mathrm{~min}$ before a solution of the intermediate enal $\mathbf{3 8}$ was added as a solution in THF $(0.5+0.5 \mathrm{~mL})$ at $-78{ }^{\circ} \mathrm{C}$. The reaction was stirred at $-78{ }^{\circ} \mathrm{C}$ for $1 \mathrm{~h}$ before being warmed to $0{ }^{\circ} \mathrm{C}$ for a further $1 \mathrm{~h}$. The reaction was quenched with acetic acid $(0.1 \mathrm{~mL})$ and then saturated aqueous $\mathrm{NaHCO}_{3}(10 \mathrm{~mL})$. The aqueous layer was separated and extracted with EtOAc $(3 \times 10 \mathrm{~mL})$. The combined organic layers were dried $\left(\mathrm{MgSO}_{4}\right)$, filtered, and concentrated in vacuo. Purification by flash column chromatography (20:1 petrol/ ethyl acetate) gave the OTMS-protected version title compound $(\mathrm{m} / \mathrm{z}$ $\left.379 \mathrm{M}+\mathrm{Na}^{+}, 100\right)$, which was then dissolved in THF $(1 \mathrm{~mL})$ and cooled to $0{ }^{\circ} \mathrm{C}$, and TBAF $(0.13 \mathrm{~mL}, 1 \mathrm{M}$ in THF, $0.13 \mathrm{mmol})$ was added and the reaction stirred for $5 \mathrm{~min}$. The reaction was quenched with saturated aqueous $\mathrm{NH}_{4} \mathrm{Cl}(1 \mathrm{~mL})$. The aqueous layer was separated and extracted with EtOAc $(3 \times 8 \mathrm{~mL})$. Purification by flash column chromatography (5:1 petrol/ethyl acetate) gave the title compound as a colorless oil $(5 \mathrm{mg}, 18 \mu \mathrm{mol}, 39 \%): R_{f} 0.40$ (5:1: petrol/ethyl acetate); $\nu_{\max } / \mathrm{cm}^{-1}$ (thin film) $3461 \mathrm{br}(\mathrm{OH}), 3296 \mathrm{~m}$ (CH), 2968m, 1442m, 1066s; ${ }^{1} \mathrm{H}$ NMR $\left(500 \mathrm{MHz} \mathrm{CDCl}_{3}\right) \delta 6.22$ (dt, $J=15.9,7.4 \mathrm{~Hz}, 1 \mathrm{H}, \mathrm{CH}_{2} \mathrm{CH}=\mathrm{CH}$ ), 5.59 (dddd, $J=15.9,2.2$, $\left.1.6,1.5 \mathrm{~Hz}, 1 \mathrm{H}, \mathrm{CH}_{2} \mathrm{CH}=\mathrm{CH}\right), 4.41(\mathrm{ddd}, J=9.1,6.8,2.6 \mathrm{~Hz}, 1 \mathrm{H}$, CHORCHOR), 4.11 (ddd, $J=9.8,3.3,2.6 \mathrm{~Hz}, 1 \mathrm{H}, \mathrm{CHORCHOR}$ ), 4.08 (ddd, $J=6.5,5.6,5.4 \mathrm{~Hz}, 1 \mathrm{H}, \mathrm{CHORCHCl}$ ), 4.05 (br m, $1 \mathrm{H}$, $\mathrm{CHOH}) 3.96$ (ddd, $J=7.8,5.4,4.2 \mathrm{~Hz}, 1 \mathrm{H}, \mathrm{CHCl}$ ), 3.54 (dt, $J=6.9$, $2.5 \mathrm{~Hz}, 1 \mathrm{H}, \mathrm{EtCHOR}), 3.00$ (br s, $1 \mathrm{H}, \mathrm{OH}), 2.83(\mathrm{~d}, J=2.2 \mathrm{~Hz}, 1 \mathrm{H}$ ), $2.40-2.53\left(\mathrm{~m}, 2 \mathrm{H}, \mathrm{CH}_{2} \mathrm{CH}=\mathrm{CH}\right), 2.25(\mathrm{ddd}, 1 \mathrm{H}, J=14.0,9.8,5.5$ $\left.\mathrm{Hz}, \mathrm{CHH}^{\prime} \mathrm{CHOH}\right), 2.18(\mathrm{ddd}, J=13.8,6.8,4.2 \mathrm{~Hz}, 1 \mathrm{H}$, $\mathrm{CHH}^{\prime} \mathrm{CHCl}$ ), 2.05 (ddd, $J=13.8,9.1,7.8 \mathrm{~Hz}, 1 \mathrm{H}, \mathrm{CH} H^{\prime} \mathrm{CHCl}$ ), $1.78\left(\mathrm{dd}, J=14.0,3.3 \mathrm{~Hz}, 1 \mathrm{H}, \mathrm{CH} H^{\prime} \mathrm{CHOH}\right.$ ), 1.70 (qn, $J=7.5 \mathrm{~Hz}$, $\left.2 \mathrm{H}, \mathrm{CH}_{3} \mathrm{CH}_{2}\right), 0.98\left(\mathrm{t}, J=7.5 \mathrm{~Hz}, 1 \mathrm{H}, \mathrm{CH}_{3}\right) ;{ }^{13} \mathrm{C}\left\{{ }^{1} \mathrm{H}\right\} \mathrm{NMR}(75$ $\left.\mathrm{MHz}, \mathrm{CDCl}_{3}\right) \delta 139.9\left(\mathrm{CH}_{2} \mathrm{CH}=\mathrm{CH}\right), 112.4\left(\mathrm{CH}=\mathrm{CH}_{2}\right), 86.0$ ( $\mathrm{CHORCHCl}), 85.7$ (EtCHOR), $81.7(\mathrm{CCH}), 79.2$ (CHORCHOR), 77.9 (CHORCHOR), $77.2(\mathrm{CCH}), 71.0(\mathrm{CHOH}), 58.2(\mathrm{CHCl})$, $38.1\left(\mathrm{CH}_{2} \mathrm{CHCl}\right), 36.5\left(\mathrm{CH}_{2} \mathrm{CH}=\mathrm{CH}_{2}\right), 35.1\left(\mathrm{CH}_{2} \mathrm{CHOH}\right), 21.7$ $\left(\mathrm{CH}_{3} \mathrm{CH}_{2}\right), 10.5\left(\mathrm{CH}_{3}\right)$; MS (ESI-TOF) $m / z 307\left[{ }^{35} \mathrm{M}+\mathrm{Na}^{+}\right], 309$ $\left[{ }^{37} \mathrm{M}+\mathrm{Na}^{+}\right]$; HRMS (ESI-TOF) $m / z[\mathrm{M}+\mathrm{Na}]^{+}$calcd for $\mathrm{C}_{15} \mathrm{H}_{21}{ }^{35} \mathrm{ClO}_{3} \mathrm{Na}$ 307.1071; found 307.1066; $[\alpha]_{\mathrm{D}}^{20}-51.9(c=0.45$ in $\left.\mathrm{CHCl}_{3}\right)$, lit. $[\alpha]_{\mathrm{D}}^{22}-67.8\left(c=0.09\right.$ in $\left.\mathrm{CHCl}_{3}\right)$.

$\left(2 R, 2^{\prime} S, 4 S, 4^{\prime} R, 5 R, 5^{\prime} S\right)-4^{\prime}$-chloro-5-ethyl-5'-((E)-pent-2-en-4-yn-1yl)octahydro-[2,2'-bifuran]-4-ol (2d). Alcohol $2 \mathrm{~b}(2 \mathrm{mg}, 7 \mu \mathrm{mol})$ and $\mathrm{PPh}_{3}(20.2 \mathrm{mg}, 77 \mu \mathrm{mol})$ were dissolved in THF $(0.3 \mathrm{~mL})$ and cooled to $0{ }^{\circ} \mathrm{C}$. DIAD $(15.6 \mu \mathrm{L}, 77 \mu \mathrm{mol})$ was added dropwise, and the reaction was stirred for $30 \mathrm{~min}$ before the addition of 4nitrobenzoic acid ( $13 \mathrm{mg}, 77 \mu \mathrm{mol})$. The reaction was then warmed to rt and stirred for $2 \mathrm{~h}$ before being concentrated in vacuo. Purification by short silica plug (DCM) gave the intermediate ester mixed with $\mathrm{DIADH}_{2}$. The mixture was then dissolved in $\mathrm{MeOH}(0.2$ $\mathrm{mL}$ ) and cooled to $0{ }^{\circ} \mathrm{C}$, and $\mathrm{K}_{2} \mathrm{CO}_{3}(5.5 \mathrm{mg}, 40 \mu \mathrm{mol})$ was added. The reaction was stirred for $30 \mathrm{~min}$, diluted with $\mathrm{H}_{2} \mathrm{O}(5 \mathrm{~mL})$, and extracted with EtOAc $(3 \times 10 \mathrm{~mL})$. The combined organic layers were then dried $\left(\mathrm{MgSO}_{4}\right)$, filtered, and concentrated in vacuo. Purification by column chromatography (3:1 petrol/ethyl acetate) gave the title compound as a colorless oil (1.8 mg, $7 \mu \mathrm{mol}, 90 \%): R_{f}$ 0.62 (3:1 petrol/ethyl acetate); $\nu_{\max } / \mathrm{cm}^{-1}$ (thin film) $3444 \mathrm{br}(\mathrm{OH})$, $3291 \mathrm{~m}(\mathrm{CH}), 2966 \mathrm{~m}, 1442 \mathrm{~m}, 1066 \mathrm{~s} ;{ }^{1} \mathrm{H}$ NMR $\left(500 \mathrm{MHz} \mathrm{CDCl}_{3}\right) \delta$ $6.25\left(\mathrm{dt}, J=16.2,7.4 \mathrm{~Hz}, 1 \mathrm{H}, \mathrm{CH}_{2} \mathrm{CH}=\mathrm{CH}\right), 5.57(\mathrm{~d} \mathrm{t}, J=16.2,1.8$ $\mathrm{Hz}, 1 \mathrm{H}, \mathrm{CH}=\mathrm{CH}), 4.08-4.15(\mathrm{~m}, 3 \mathrm{H}, \mathrm{CHORCHOR}, \mathrm{CHOH})$, 3.97-4.02 (m, 2H, CHClCHOR), $3.70(\mathrm{td}, J=6.7,3.1 \mathrm{~Hz}, 1 \mathrm{H}$, CHEt), $2.83(\mathrm{~d}, J=1.8 \mathrm{~Hz}, 1 \mathrm{H}, \mathrm{CCH}), 2.43-2.49(\mathrm{~m}, 2 \mathrm{H}$, $\left.\mathrm{CHH}^{\prime} \mathrm{CH}=\mathrm{CH}\right), 2.34-2.40\left(\mathrm{~m}, 1 \mathrm{H}, \mathrm{CH} H^{\prime} \mathrm{CH}=\mathrm{CH}\right), 2.26-2.32$ $\left(\mathrm{m}, 1 \mathrm{H}, \mathrm{CH} \mathrm{H}^{\prime} \mathrm{CHCl}\right), 2.19$ (ddd, $J=13.4,6.4,4.7 \mathrm{~Hz}, 1 \mathrm{H}$, $\mathrm{CHH}^{\prime} \mathrm{CHCl}$ ), 1.95 (ddd, $\left.J=13.3,5.9,2.7 \mathrm{~Hz}, 1 \mathrm{H}, \mathrm{CHOHCHH}^{\prime}\right)$, $1.86\left(\mathrm{ddd}, J=13.2,8.8,6.2 \mathrm{~Hz}, 1 \mathrm{H}, \mathrm{CHOHCHH}{ }^{\prime}\right), 1.46-1.56(\mathrm{~m}$, $\left.2 \mathrm{H}, \mathrm{CH}_{2} \mathrm{Me}\right), 0.97(\mathrm{t}, J=7.4 \mathrm{~Hz}, 3 \mathrm{H}, \mathrm{Me}) ;{ }^{13} \mathrm{C}\left\{{ }^{1} \mathrm{H}\right\} \mathrm{NMR}(125 \mathrm{MHz}$ $\left.\mathrm{CDCl}_{3}\right) \delta 140.9\left(\mathrm{CH}_{2} \mathrm{CH}=\mathrm{CH}\right), 111.7(\mathrm{CH}=\mathrm{CH}), 88.2(\mathrm{CHEt})$, 85.7 (CHClCHOR), 81.9 (CCH), 79.9, (CHORCHOR), 79.1 (CHORCHOR), $76.6(\mathrm{CCH}), 75.7(\mathrm{CHOH}), 58.9(\mathrm{CHCl}), 37.8$ $\left(\mathrm{CH}_{2} \mathrm{CHCl}\right), 37.6\left(\mathrm{CHOHCH}_{2}\right), 36.8\left(\mathrm{CH}_{2} \mathrm{CH}=\mathrm{CH}\right), 27.0$ $\left(\mathrm{CH}_{2} \mathrm{Me}\right.$ ), $10.2(\mathrm{Me})$; HRMS (ESI-TOF) $\mathrm{m} / z[\mathrm{M}+\mathrm{Na}]^{+}$calcd for $\mathrm{C}_{15} \mathrm{H}_{21} \mathrm{ClO}_{3} \mathrm{Na}$ 307.1071; found 307.1070; $[\alpha]_{\mathrm{D}}^{20}-31.3(c=0.16$ in $\mathrm{CHCl}_{3}$ ).

Notoryne: Oxford Route. $\left(2 S, 2^{\prime} R, 4 S, 4^{\prime} S, 5 R, 5^{\prime} R\right)-5^{\prime}-A l l y l-4^{\prime}$ chloro-5-ethyloctahydro-[2,2'-bifuran]-4-ol. To a stirred solution of ether $28(35 \mathrm{mg}, 0.09 \mathrm{mmol})$ in DCM $(9 \mathrm{~mL})$ was added $\mathrm{BCl}_{3}$. $\mathrm{SMe}_{2}(0.23 \mathrm{~mL}, 2 \mathrm{M}$ in DCM, $0.45 \mathrm{mmol})$, and the reaction was stirred for $5 \mathrm{~min}$. The reaction was quenched with saturated aqueous $\mathrm{NaHCO}_{3}(6 \mathrm{~mL})$ and the stirred vigorously for $10 \mathrm{~min}$. The aqueous layer was separated and extracted with DCM $(3 \times 10 \mathrm{~mL})$. The combined organic layers were dried $\left(\mathrm{Na}_{2} \mathrm{SO}_{4}\right)$, filtered, and concentrated in vacuo. Purification by flash column chromatography (3:1 petrol/ethyl acetate) gave the title compound as a colorless oil (22 mg, $86 \mu \mathrm{mol}, 95 \%): R_{f} 0.56$ (3:1 petrol/ethyl acetate); $\nu_{\max } / \mathrm{cm}^{-1}$ (thin film) 3432br (OH), 2964s, 1642w,1438w, 1074s, 921m; ${ }^{1} \mathrm{H}$ $\operatorname{NMR}\left(500 \mathrm{MHz} \mathrm{CDCl}_{3}\right) \delta 5.82(\mathrm{ddt}, J=17.2,10.1,6.8 \mathrm{~Hz}, 1 \mathrm{H}$, $\left.\mathrm{CH}=\mathrm{CH}_{2}\right), 5.17\left(\mathrm{dd}, J=17.2,1.6 \mathrm{~Hz}, 1 \mathrm{H}, \mathrm{CH}=\mathrm{CHH}^{\prime}\right), 5.14(\mathrm{dd}, J$ 
$\left.=10.1,1.6 \mathrm{~Hz}, 1 \mathrm{H}, \mathrm{CH}=\mathrm{CHH}^{\prime}\right), 4.42(\mathrm{ddd}, J=9.3,6.4,2.6 \mathrm{~Hz}, 1 \mathrm{H}$, CHORCHOR), 4.19 (dt, $J=9.3,3.1 \mathrm{~Hz}, 1 \mathrm{H}, \mathrm{CHORCHOR}), 4.12$ $(\mathrm{dt}, J=6.6,5.3 \mathrm{~Hz}, 1 \mathrm{H}, \mathrm{CHORCHCl}), 3.99-4.04(\mathrm{~m}, 2 \mathrm{H}, \mathrm{CHCl}$, $\mathrm{CHOH}), 3.88(\mathrm{td}, J=7.4,1.0 \mathrm{~Hz}, 1 \mathrm{H}, \mathrm{EtCHOR}), 3.43($ br s, $1 \mathrm{H}$, $\mathrm{OH}), 2.41-2.47\left(\mathrm{~m}, 1 \mathrm{H}, \mathrm{CHHCH}=\mathrm{CH}_{2}\right), 2.33-2.39(\mathrm{~m}, 1 \mathrm{H}$, $\left.\mathrm{CHHCH}=\mathrm{CH}_{2}\right), 2.28(\mathrm{ddd}, J=13.9,6.4,3.8 \mathrm{~Hz}, 1 \mathrm{H}$, $\mathrm{CHH}^{\prime} \mathrm{CHOH}$ ), 2.18 (ddd, $J=13.9,6.4,2.6 \mathrm{~Hz}, 1 \mathrm{H}, \mathrm{CH} \mathrm{H}^{\prime} \mathrm{CHCl}$ ), $2.04\left(\mathrm{ddd}, J=13.9,9.3,8.0 \mathrm{~Hz}, 1 \mathrm{H}, \mathrm{CHH} \mathrm{CHCl}^{\prime}\right), 1.78$ (ddd, $J=$ 13.9, 3.4, $1.6 \mathrm{~Hz}, 1 \mathrm{H}, \mathrm{CH} H^{\prime} \mathrm{CHOH}$ ), 1.38 (qn, $J=7.4 \mathrm{~Hz}, 2 \mathrm{H}$, $\left.\mathrm{CH}_{3} \mathrm{CH}_{2}\right), 0.95\left(\mathrm{t}, J=7.4 \mathrm{~Hz}, 3 \mathrm{H}, \mathrm{CH}_{3}\right) ;{ }^{13} \mathrm{C}\left\{{ }^{1} \mathrm{H}\right\} \mathrm{NMR}(125 \mathrm{MHz}$, $\left.\mathrm{CDCl}_{3}\right) \delta 132.8\left(\mathrm{CH}=\mathrm{CH}_{2}\right), 118.5\left(\mathrm{CH}=\mathrm{CH}_{2}\right), 89.1(\mathrm{EtCHOR})$, 86.7 (CHORCHCl), 79.7 (CHORCHOR), 78.2 (CHORCHOR), $74.8(\mathrm{CHOH}), 58.5(\mathrm{CHCl}), 38.3\left(\mathrm{CH}_{2} \mathrm{CHCl}\right), 37.6\left(\mathrm{CH}_{2} \mathrm{CH}=\right.$ $\left.\mathrm{CH}_{2}\right)$, $33.8\left(\mathrm{CH}_{2} \mathrm{CHOH}\right), 26.1\left(\mathrm{CH}_{3} \mathrm{CH}_{2}\right), 10.2\left(\mathrm{CH}_{3}\right)$; MS (ESITOF) $m / z 283\left[{ }^{35} \mathrm{M}+\mathrm{Na}^{+}\right], 285\left[{ }^{37} \mathrm{M}+\mathrm{Na}^{+}\right]$; HRMS (ESI-TOF) $m / z[\mathrm{M}+\mathrm{Na}]^{+}$calcd for $\mathrm{C}_{13} \mathrm{H}_{21} \mathrm{NO}_{3}{ }^{35} \mathrm{ClNa}$ 283.1071; found 283.1072; $[\alpha]_{\mathrm{D}}^{20}+60.4\left(c=1.0\right.$ in $\left.\mathrm{CHCl}_{3}\right)$.

$\left(2 S, 2^{\prime} R, 4 R, 4^{\prime} S, 5 R, 5^{\prime} R\right)-5^{\prime}$-Allyl-4' -chloro-5-ethyloctahydro-[2,2' bifuran]-4-yl 4-nitrobenzoate. To a stirred solution of $\left(2 S, 2^{\prime} R, 4 S, 4^{\prime} S, 5 R, 5^{\prime} R\right)-5^{\prime}$-allyl-4' -chloro-5-ethyloctahydro-[2,2' -bifuran]-4-ol (16 mg, $62 \mu \mathrm{mol})$ and $\mathrm{PPh}_{3}(78 \mathrm{mg}, 0.3 \mathrm{mmol})$ in THF $(0.9$ $\mathrm{mL})$ at $0{ }^{\circ} \mathrm{C}$ was added DIAD $(60 \mu \mathrm{L}, 60 \mu \mathrm{g}, 0.3 \mathrm{mmol})$. The reaction mixture was stirred for $30 \mathrm{~min}$ at $0{ }^{\circ} \mathrm{C}$, during which time a white precipitate formed. 4-Nitrobenzoic acid $(50 \mathrm{mg}, 0.3 \mathrm{mmol})$ was added in one batch, and the reaction was stirred for $1 \mathrm{~h}$. The reaction was then concentrated in vacuo and purified by flash column chromatography (DCM) to give the title compound as a colorless oil (19 mg, $46 \mu \mathrm{mol}, 74 \%): R_{f} 0.45\left(8: 1\right.$ petrol/acetone); $\nu_{\max } / \mathrm{cm}^{-1}$ (thin film) $2967 \mathrm{w}, 1725 \mathrm{~s}, 1529 \mathrm{~s}, 1348 \mathrm{w}, 1274 \mathrm{~s}, 1103 \mathrm{~m}$; ${ }^{1} \mathrm{H}$ NMR (400 $\left.\mathrm{MHz} \mathrm{CDCl}_{3}\right) \delta 8.30(\mathrm{dd}, J=7.0,1.9 \mathrm{~Hz}, 2 \mathrm{H}, \mathrm{ArH}), 8.22(\mathrm{dd}, J=7.0$, $1.9 \mathrm{~Hz}, 2 \mathrm{H}, \mathrm{ArH}), 5.83\left(\mathrm{ddt}, J=17.0,10.0,6.7 \mathrm{~Hz}, 1 \mathrm{H}, \mathrm{CH}=\mathrm{CH}_{2}\right.$ ), $5.63(\mathrm{t}, J=3.4 \mathrm{~Hz}, 1 \mathrm{H}, \mathrm{CHOPNB}), 5.13(\mathrm{dd}, J=17.0,0.9 \mathrm{~Hz}, 1 \mathrm{H}$, $\left.\mathrm{CH}=\mathrm{CHH}^{\prime}\right), 5.12\left(\mathrm{dd}, J=10.0,0.9 \mathrm{~Hz}, 1 \mathrm{H}, \mathrm{CH}=\mathrm{CHH}^{\prime}\right), 4.22-$ 4.30 (m, 2H, CHORCHOR), 4.05-4.09 (m, 2H, CHClCHOR), 4.02 $(\mathrm{td}, J=7.1,3.4 \mathrm{~Hz}, 1 \mathrm{H}, \mathrm{EtCHOR}), 2.32-2.39\left(\mathrm{~m}, 2 \mathrm{H}, \mathrm{CH}_{2} \mathrm{CH}=\right.$ $\mathrm{CH}_{2}$ ), 2.20-2.28 (m, 4H, CH $\left.\mathrm{CHOPNB}_{2} \mathrm{CH}_{2} \mathrm{CHCl}\right), 1.57-1.80(\mathrm{~m}$, $\left.2 \mathrm{H}, \mathrm{CH}_{3} \mathrm{CH}_{2}\right), 0.97\left(\mathrm{t}, J=7.5 \mathrm{~Hz}, 3 \mathrm{H}, \mathrm{CH}_{3}\right) ;{ }^{13} \mathrm{C}\left\{{ }^{1} \mathrm{H}\right\} \mathrm{NMR}(100$ $\left.\mathrm{MHz}, \mathrm{CDCl}_{3}\right) \delta 164.0(\mathrm{C}=\mathrm{O}), 150.7(\mathrm{Ar}), 135.3(\mathrm{Ar}), 133.3(\mathrm{CH}=$ $\left.\mathrm{CH}_{2}\right), 130.8(\mathrm{Ar}), 123.6(\mathrm{Ar}), 118.0\left(\mathrm{CH}=\mathrm{CH}_{2}\right), 86.5$ (CHORCHCl), 83.4 (EtCHOR), 80.0, 78.8 (CHORCHOR), 76.6 (CHOPNB), $59.0(\mathrm{CHCl}), 38.1\left(\mathrm{CH}_{2}\right), 37.9\left(\mathrm{CH}_{2} \mathrm{CH}=\mathrm{CH}_{2}\right), 35.6$ $\left(\mathrm{CH}_{2}\right), 22.6\left(\mathrm{CH}_{3} \mathrm{CH}_{2}\right), 10.5\left(\mathrm{CH}_{3}\right)$; MS (ESI-TOF) $\mathrm{m} / z 432\left[{ }^{35} \mathrm{M}+\right.$ $\left.\mathrm{Na}^{+}\right], 434\left[{ }^{37} \mathrm{M}+\mathrm{Na}^{+}\right]$; HRMS (ESI-TOF) $m / z[\mathrm{M}+\mathrm{Na}]^{+}$calcd for $\mathrm{C}_{20} \mathrm{H}_{24} \mathrm{NO}_{6}{ }^{35} \mathrm{ClNa}$ 432.1184; found 432.1185; $[\alpha]_{\mathrm{D}}^{20}-4.0(c=1.0$ in $\left.\mathrm{CHCl}_{3}\right)$.

$\left(2 S, 2^{\prime} R, 4 R, 4^{\prime} S, 5 R, 5^{\prime} R\right)-5^{\prime}$-Allyl-4' -chloro-5-ethyloctahydro-[2, $2^{\prime}$ bifuran]-4-ol (10). To a stirred solution of $\left(2 S, 2^{\prime} R, 4 R, 4^{\prime} S, 5 R, 5^{\prime} R\right)-5^{\prime}-$ allyl-4' -chloro-5-ethyloctahydro-[2,2'-bifuran]-4-yl 4-nitrobenzoate $(19 \mathrm{mg}, 46 \mu \mathrm{mol})$ in $\mathrm{MeOH}(1 \mathrm{~mL})$ was added $\mathrm{K}_{2} \mathrm{CO}_{3}(39 \mathrm{mg}$, $132 \mathrm{mmol}$ ), and the reaction was stirred for $30 \mathrm{~min}$. The mixture was diluted with $\mathrm{H}_{2} \mathrm{O}(3 \mathrm{~mL})$ and the aqueous layer extracted with EtOAc $(3 \times 10 \mathrm{~mL})$. The combined organic layers were dried $\left(\mathrm{MgSO}_{4}\right)$, filtered, and concentrated in vacuo. Purification by flash column chromatography ( $3: 1$ petrol/ethyl acetate) gave the title compound as a colorless oil (11 mg, $42 \mu \mathrm{mol}, 91 \%): R_{f}(0.543: 1$ petrol/ethyl acetate); $v_{\max } / \mathrm{cm}^{-1}$ (thin film) $3247 \mathrm{br}(\mathrm{OH}), 2932 \mathrm{~s}, 1642 \mathrm{w}, 1434 \mathrm{w}$, 1055s, 1013m, 924s; ${ }^{1} \mathrm{H}$ NMR (500 $\mathrm{MHz} \mathrm{CDCl}_{3}$ ) $\delta 5.83$ (ddt, $J=$ $\left.17.1,10.3,7.0 \mathrm{~Hz}, 1 \mathrm{H}, \mathrm{CH}=\mathrm{CH}_{2}\right), 5.14(\mathrm{dd}, J=17.1,1.5 \mathrm{~Hz}, 1 \mathrm{H}$, $\left.\mathrm{CH}=\mathrm{CHH}^{\prime}\right), 5.11\left(\mathrm{dd}, J=10.3,1.5 \mathrm{~Hz}, 1 \mathrm{H}, \mathrm{CH}=\mathrm{CHH}^{\prime}\right), 4.28-$ $4.31(\mathrm{~m}, 1 \mathrm{H}, \mathrm{CHOH}), 4.16-4.22(\mathrm{~m}, 2 \mathrm{H}, \mathrm{CHORCHOR}), 4.02-4.07$ (m, 2H, CHClCHOR), $3.73(\mathrm{td}, J=7.3,2.8 \mathrm{~Hz}, 1 \mathrm{H}, \mathrm{EtCHOR})$, $2.28-2.42\left(\mathrm{~m}, 2 \mathrm{H}, \mathrm{CH}_{2} \mathrm{CH}=\mathrm{CH}_{2}\right), 2.16-2.24\left(\mathrm{~m}, 2 \mathrm{H}, \mathrm{CH}_{2} \mathrm{CHCl}\right)$, 2.10 (ddd, $\left.J=13.0,5.6,1.0 \mathrm{~Hz}, 1 \mathrm{H}, \mathrm{CHH}^{\prime} \mathrm{CHOH}\right), 1.97$ (ddd, $J=$ 13.0, 8.7, $4.7 \mathrm{~Hz}, 1 \mathrm{H}, \mathrm{CHH} \mathrm{CHOH}^{\prime}$, 1.57-1.74 (m, $\left.2 \mathrm{H}, \mathrm{CH}_{3} \mathrm{CH}_{2}\right)$, $1.45(\mathrm{~d}, J=5.3 \mathrm{~Hz}, 1 \mathrm{H}, \mathrm{OH}), 0.99\left(\mathrm{t}, J=7.5 \mathrm{~Hz}, 3 \mathrm{H}, \mathrm{CH}_{3}\right) ;{ }^{13} \mathrm{C}\left\{{ }^{1} \mathrm{H}\right\}$ NMR $\left(125 \mathrm{MHz}, \mathrm{CDCl}_{3}\right) \delta 133.4\left(\mathrm{CH}=\mathrm{CH}_{2}\right), 117.9\left(\mathrm{CH}=\mathrm{CH}_{2}\right)$, 86.4 (CHORCHCl), 84.6 (EtCHOR), 80.3 (CHORCHOR), 78.4 (CHORCHOR), $72.7(\mathrm{CHOH}), 59.1(\mathrm{CHCl}), 38.1\left(\mathrm{CH}_{2} \mathrm{CHCl}\right)$, $37.9\left(\mathrm{CH}_{2} \mathrm{CH}=\mathrm{CH}_{2}, \mathrm{CH}_{2} \mathrm{CHOH}\right), 22.0\left(\mathrm{CH}_{3} \mathrm{CH}_{2}\right), 10.4\left(\mathrm{CH}_{3}\right)$;
HRMS (ESI-TOF) $m / z[\mathrm{M}+\mathrm{Na}]^{+}$calcd for $\mathrm{C}_{13} \mathrm{H}_{21} \mathrm{O}_{3} \mathrm{ClNa}$ 283.1071; found 283.1071; $[\alpha]_{\mathrm{D}}^{20}+6.8\left(c=1.0\right.$ in $\left.\mathrm{CHCl}_{3}\right)$.

$\left(2 R, 2^{\prime} S, 4 S, 4^{\prime} S, 5 R, 5^{\prime} R\right)-5$-Allyl-4' -bromo-4-chloro-5' -ethyloctahydro-2,2'-bifuran (47-Oxford). To a stirred solution of alcohol 10 (14 $\mathrm{mg}, 57 \mu \mathrm{mol})$ in toluene $(2.4 \mathrm{~mL})$ were added $\mathrm{PPh}_{3}(78 \mathrm{mg}, 0.3$ $\mathrm{mmol})$ and $\mathrm{CBr}_{4}((99 \mathrm{mg}, 0.3 \mathrm{mmol})$, which was then purified by sublimation, dissolved in DCM, filtered through deactivated alumina, and dried in a desiccator over $\mathrm{KOH}$ for $16 \mathrm{~h}$ ), and the mixture was heated to $80^{\circ} \mathrm{C}$ for $75 \mathrm{~min}$. The reaction mixture was cooled to rt and then diluted with DCM $(1 \mathrm{~mL})$ and loaded directly onto a column. Purification by flash column chromatography (DCM) gave the title compound as a colorless oil $(14 \mathrm{mg}, 43 \mu \mathrm{mol}, 75 \%): R_{f} 0.82(5: 1$ petrol/ethyl acetate); $\nu_{\max } / \mathrm{cm}^{-1}$ (thin film) $2964 \mathrm{~m}, 1456 \mathrm{~m}, 1262 \mathrm{~m}$, $1067 \mathrm{~s} ;{ }^{1} \mathrm{H}$ NMR $\left(500 \mathrm{MHz} \mathrm{CDCl}_{3}\right) \delta 5.83(\mathrm{ddt}, J=17.0,10.1,6.9$ $\left.\mathrm{Hz}, 1 \mathrm{H}, \mathrm{CH}=\mathrm{CH}_{2}\right), 5.15\left(\mathrm{dd}, J=17.0,1.6 \mathrm{~Hz}, 1 \mathrm{H}, \mathrm{CH}=\mathrm{CHH}^{\prime}\right)$, $5.12\left(\mathrm{dd}, J=10.1,1.6 \mathrm{~Hz}, 1 \mathrm{H}, \mathrm{CH}=\mathrm{CHH}^{\prime}\right), 4.24(\mathrm{dt}, J=7.2,5.5 \mathrm{~Hz}$, $1 \mathrm{H}, \mathrm{CHORCHOR}), 4.03-4.07(\mathrm{~m}, 2 \mathrm{H}, \mathrm{CHCl}, \mathrm{CHORCHCl}), 3.97$ (ddd, $J=8,2,6.9,5.5 \mathrm{~Hz}, 1 \mathrm{H}, \mathrm{CHORCHOR}), 3.91(\mathrm{td}, J=7.5,4.0$ $\mathrm{Hz}, 1 \mathrm{H}, \mathrm{EtCHOR}), 3.86(\mathrm{td}, J=8.7,7.3 \mathrm{~Hz}, 1 \mathrm{H}, \mathrm{CHBr}), 2.66(\mathrm{dt}, J=$ 13.1, $\left.6.9 \mathrm{~Hz}, 1 \mathrm{H}, \mathrm{CHH} \mathrm{H}^{\prime} \mathrm{CHBr}\right), 2.31-2.40\left(\mathrm{~m}, 2 \mathrm{H}, \mathrm{CH}_{2} \mathrm{CH}=\mathrm{CH}_{2}\right)$, 2.24-2.26 (m, $\left.2 \mathrm{H}, \mathrm{CH}_{2} \mathrm{CHCl}\right), 2.18(\mathrm{dt}, J=13.6,8.0 \mathrm{~Hz}, 1 \mathrm{H}$, $\left.\mathrm{CH} H^{\prime} \mathrm{CHBr}\right), 1.76\left(\mathrm{dqd}, J=13.8,7.5,3.8 \mathrm{~Hz}, 1 \mathrm{H}, \mathrm{CH}_{3} \mathrm{CHH}^{\prime}\right), 1.50$ (dqn, $\left.J=13.8,7.5 \mathrm{~Hz}, 1 \mathrm{H}, \mathrm{CH}_{3} \mathrm{CHH}^{\prime}\right), 1.01(\mathrm{t}, J=7.5 \mathrm{~Hz}, 3 \mathrm{H}$, $\left.\mathrm{CH}_{3}\right) ;{ }^{13} \mathrm{C}\left\{{ }^{1} \mathrm{H}\right\}$ NMR $\left(125 \mathrm{MHz}, \mathrm{CDCl}_{3}\right) \delta 133.3\left(\mathrm{CH}=\mathrm{CH}_{2}\right)$, 118.0 $\left(\mathrm{CH}=\mathrm{CH}_{2}\right), 87.1$ (EtCHOR), 86.4 (CHORCHCl), 79.9 (CHORCHOR), 78.9 (CHORCHOR), 58.9 ( $\mathrm{CHCl}), 47.3$ (CHBr), $39.3\left(\mathrm{CH}_{2} \mathrm{CHCl}\right), 38.0\left(\mathrm{CH}_{2} \mathrm{CHBr}\right), 37.8\left(\mathrm{CH}_{2} \mathrm{CH}=\mathrm{CH}_{2}\right), 25.4$ $\left(\mathrm{CH}_{3} \mathrm{CH}_{2}\right), 10.0\left(\mathrm{CH}_{3}\right)$; MS (ESI-TOF) $m / z 345\left[\mathrm{M}+\mathrm{Na}^{+}\right], 347[\mathrm{M}$ $\left.+\mathrm{Na}^{+}\right]$; HRMS (ESI-TOF) $m / z[\mathrm{M}+\mathrm{Na}]^{+}$calcd for $\mathrm{C}_{13} \mathrm{H}_{20} \mathrm{O}_{2}{ }^{35} \mathrm{Cl}^{79} \mathrm{BrNa}$ 345.0227; found 345.0235; $[\alpha]_{\mathrm{D}}^{20}+29.5(c=$ 1.0 in $\left.\mathrm{CHCl}_{3}\right)$.

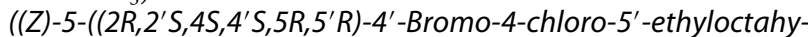
dro-[2,2'-bifuran]-5-yl)pent-3-en-1-yn-1-yl)trimethylsilane (48). A mixture of ozone and oxygen was gently bubbled through a stirred solution of 47 -Oxford $(5 \mathrm{mg}, 15 \mu \mathrm{mol})$ in DCM $(3 \mathrm{~mL})$ at $-78{ }^{\circ} \mathrm{C}$ until the solution became pale blue (approximately $2 \mathrm{~min}$ ). The excess ozone was purged from the solution by bubbling oxygen through for a further $5 \mathrm{~min}$. Triphenylphosphine $(20 \mathrm{mg}, 75 \mu \mathrm{mol})$ was added, and the reaction mixture was allowed to warm to $\mathrm{rt}$ over $15 \mathrm{~h}$. The reaction mixture was dry-loaded onto silica and rapidly purified by flash column chromatography $\left(5: 1\right.$ petrol bp $30-40{ }^{\circ} \mathrm{C}$ / diethyl ether) to give the corresponding aldehyde as a colorless oil (4.4 $\mathrm{mg}, 13.6 \mu \mathrm{mol}, 91 \%)$ that was used in the subsequent transformation without characterization.

To a solution of 3-(tert-butyldimethylsilyl)-1-trimethylsilanylpropyne $(135 \mathrm{mg}, 0.63 \mathrm{mmol})$ in dry THF $(1 \mathrm{~mL})$ at $-78{ }^{\circ} \mathrm{C}$ was added dropwise tert-butyllithium $(0.37 \mathrm{~mL}, 1.7 \mathrm{M}$ in pentane, 0.63 $\mathrm{mmol}$ ), and this solution was stirred at $-78^{\circ} \mathrm{C}$ for $1 \mathrm{~h}$. A solution of titanium(IV) isopropoxide $(0.19 \mathrm{~mL}, 180 \mathrm{mg}, 0.63 \mathrm{mmol})$ in dry THF $(0.5 \mathrm{~mL})$ was added dropwise to the reaction mixture; the resulting solution was stirred for $10 \mathrm{~min}$, and then $1.94 \mathrm{~mL}$ was removed and discarded. To the remaining $0.12 \mathrm{~mL}(36 \mu \mathrm{mol})$ was added dropwise a solution of the aldehyde prepared above in dry THF $(0.5 \mathrm{~mL}+0.5 \mathrm{~mL}$ rinse $)$. The reaction mixture was stirred at $-78{ }^{\circ} \mathrm{C}$ for $30 \mathrm{~min}$, and then the cooling bath was removed and the reaction mixture stirred at $\mathrm{rt}$ for $30 \mathrm{~min}$. The reaction mixture was then poured into a separating funnel containing $0.1 \mathrm{M}$ aqueous $\mathrm{HCl}$ $(2 \mathrm{~mL})$, and the aqueous layer was extracted with diethyl ether $(3 \times 5$ $\mathrm{mL})$. The combined organic layers were dried $\left(\mathrm{MgSO}_{4}\right)$, filtered, and concentrated in vacuo. Purification by flash column chromatography (50:1 petrol bp $30-40{ }^{\circ} \mathrm{C} /$ diethyl ether) gave the title compound as a colorless oil (2 mg, $4.8 \mu \mathrm{mol}, 32 \%$ from 47-Oxford, $>30: 1(Z):(E)$ from crude ${ }^{1} \mathrm{H}$ NMR analysis and characterized as such a mixture): $\nu_{\max } / \mathrm{cm}^{-1}$ (thin film) $2963 ;{ }^{1} \mathrm{H} \mathrm{NMR}\left(500 \mathrm{MHz}, \mathrm{CDCl}_{3}\right) \delta 6.02(\mathrm{dt}$, $\left.J=10.9,7.5 \mathrm{~Hz}, 1 \mathrm{HCH}_{2} \mathrm{CH}=\mathrm{CH}\right), 5.63(\mathrm{dt}, J=10.9,1.3 \mathrm{~Hz}, 1 \mathrm{H}$, $\left.\mathrm{CH}_{2} \mathrm{CH}=\mathrm{CH}\right), 4.25\left(\mathrm{td}, J=7.3,5.6 \mathrm{~Hz}, 1 \mathrm{H}, \mathrm{CHClCH}_{2} \mathrm{CHO}\right)$, 4.13-4.08 (m, $\left.2 \mathrm{H}, \mathrm{CHCH}_{2} \mathrm{CH}=\mathrm{CH}, \mathrm{CHCl}\right), 3.97$ (ddd, $J=8.3,6.7$, $\left.5.5 \mathrm{~Hz}, 1 \mathrm{H}, \mathrm{CHBrCH}_{2} \mathrm{CHO}\right), 3.91(\mathrm{td}, J=7.5,4.0 \mathrm{~Hz}, 1 \mathrm{H}, \mathrm{CHEt})$, $3.86(\mathrm{dt}, J=8.7,7.3 \mathrm{~Hz}, 1 \mathrm{H}, \mathrm{CHBr}), 2.66(\mathrm{dt}, J=13.2,6.7 \mathrm{~Hz}, 1 \mathrm{H}$, $\mathrm{CHBrCHH}), 2.69-2.63(\mathrm{~m}, 1 \mathrm{H}, \mathrm{CHHCH}=\mathrm{CH}), 2.61-2.55(\mathrm{~m}$, 
$1 \mathrm{H}, \mathrm{CHHCH}=\mathrm{CH}), 2.29-2.22\left(\mathrm{~m}, 2 \mathrm{H}, \mathrm{CHClCH}_{2}\right), 2.18(\mathrm{dt}, J=$ 13.2, $8.3 \mathrm{~Hz}, 1 \mathrm{H}, \mathrm{CHBrCHH}), 1.75(\mathrm{dqd}, J=13.9,7.4,4.0 \mathrm{~Hz}, 1 \mathrm{H}$, $\left.\mathrm{CHHCH}_{3}\right), 1.49\left(\mathrm{dqn}, J=14.0,7.4 \mathrm{~Hz}, 1 \mathrm{H}, \mathrm{CHHCH}_{3}\right), 1.00(\mathrm{t}, J=$ $\left.7.4 \mathrm{~Hz}, 3 \mathrm{H}, \mathrm{CH}_{2} \mathrm{CH}_{3}\right), 0.20\left(\mathrm{~s}, 9 \mathrm{H}, \mathrm{Si}\left(\mathrm{CH}_{3}\right)_{3}\right) ;{ }^{13} \mathrm{C}\left\{{ }^{1} \mathrm{H}\right\}$ NMR $(125$ $\left.\mathrm{MHz}, \mathrm{CDCl}_{3}\right) \delta 139.3\left(\mathrm{CH}_{2} \mathrm{CH}=\mathrm{CH}\right), 112.4\left(\mathrm{CH}_{2} \mathrm{CH}=\mathrm{CH}\right)$, $101.6(\mathrm{C} \equiv \mathrm{C}), 100.0(\mathrm{C} \equiv \mathrm{C}), 87.3(\mathrm{CHEt}), 86.5\left(\mathrm{CHCH}_{2} \mathrm{CH}=\mathrm{CH}\right)$, $80.3\left(\mathrm{CHClCH}_{2} \mathrm{CH}\right), 79.1\left(\mathrm{CHBrCH}_{2} \mathrm{CH}\right), 59.4(\mathrm{CHCl}), 47.5$ $(\mathrm{CHBr}), 39.5\left(\mathrm{CHBrCH}_{2}\right), 38.3\left(\mathrm{CHClCH}_{2}\right), 34.6\left(\mathrm{CH}_{2} \mathrm{CH}=\mathrm{CH}\right)$, $25.6\left(\mathrm{CH}_{2} \mathrm{CH}_{3}\right), 10.2\left(\mathrm{CH}_{2} \mathrm{CH}_{3}\right), 0.11\left(\mathrm{Si}\left(\mathrm{CH}_{3}\right)_{3}\right) ;[\alpha]_{\mathrm{D}}^{25}+37.5(c=$ 0.16 in $\left.\mathrm{CHCl}_{3}\right)$.

$\left(2 S, 2^{\prime} R, 4 S, 4^{\prime} S, 5 R, 5^{\prime} R\right)-4-B r o m o-4^{\prime}$-chloro-5-ethyl-5' -((Z)-pent-2en-4-yn-1-yl)octahydro-2,2'-bifuran, (Z)-notoryne ((Z)-3a). To a stirred solution of $48(2 \mathrm{mg}, 4.8 \mu \mathrm{mol})$ in THF $(1 \mathrm{~mL})$ at $-20{ }^{\circ} \mathrm{C}$ was added TBAF $(20 \mu \mathrm{L}$ of a $2.0 \mathrm{M}$ solution in THF, $40 \mu \mathrm{mol})$, and stirring was continued for $5 \mathrm{~min}$. The reaction mixture was quenched by the addition of aqueous ammonium chloride $(3 \mathrm{~mL})$, and the aqueous phase was extracted with ether $(3 \times 5 \mathrm{~mL})$. The combined organic extracts were dried $\left(\mathrm{Na}_{2} \mathrm{SO}_{4}\right)$. Purification by flash chromatography (30:1 petrol bp $30-40{ }^{\circ} \mathrm{C} /$ diethyl ether) gave the title compound as a colorless oil $\left(1.7 \mathrm{mg}, 4.8 \mu \mathrm{mol}\right.$, quant.): $\nu_{\max } /$ $\mathrm{cm}^{-1}$ (thin film) 3294, 1728, 1460, 1289, 1071, 966; ${ }^{1} \mathrm{H}$ NMR (500 $\left.\mathrm{MHz}, \mathrm{CDCl}_{3}\right) \delta 6.08\left(\mathrm{dtd}, J=10.9,7.5,0.9 \mathrm{~Hz}, 1 \mathrm{H}, \mathrm{CH}_{2} \mathrm{CH}=\mathrm{CH}\right)$, $5.60\left(\mathrm{ddt}, J=10.9,2.7,1.4 \mathrm{~Hz}, 1 \mathrm{H}, \mathrm{CH}_{2} \mathrm{CH}=\mathrm{CH}\right), 4.25(\mathrm{td}, J=7.3$, $\left.5.6 \mathrm{~Hz}, 1 \mathrm{H}, \mathrm{CHClCH}_{2} \mathrm{CHO}\right), 4.11-4.07\left(\mathrm{~m}, 2 \mathrm{H}, \mathrm{CHCH}_{2} \mathrm{CH}=\mathrm{CH}\right.$, $\mathrm{CHCl}$ ), 3.98 (ddd, $J=8.3,6.8,5.6 \mathrm{~Hz}, 1 \mathrm{H}, \mathrm{CHBrCH}_{2} \mathrm{CHO}$ ), 3.91 $(\mathrm{td}, J=7.5,3.9 \mathrm{~Hz}, 1 \mathrm{H}, \mathrm{CHEt}), 3.86(\mathrm{dt}, J=8.8,7.3 \mathrm{~Hz}, 1 \mathrm{H}, \mathrm{CHBr})$, $3.12(\mathrm{dd}, J=2.2,0.9 \mathrm{~Hz}, 1 \mathrm{H}, \mathrm{CCH}), 2.66(\mathrm{dt}, J=13.1,7.0 \mathrm{~Hz}, 1 \mathrm{H}$, $\mathrm{CHBrCHH}), 2.69-2.64(\mathrm{~m}, 1 \mathrm{H}, \mathrm{CHHCH}=\mathrm{CH}), 2.60$ (dddd, $J=$ 14.6, 7.4, 6.0, $1.4 \mathrm{~Hz}, 1 \mathrm{H}, \mathrm{CHHCH}=\mathrm{CH}), 2.29-2.24(\mathrm{~m}, 2 \mathrm{H}$, $\left.\mathrm{CHClCH}_{2}\right), 2.17(\mathrm{dt}, J=13.2,8.5 \mathrm{~Hz}, 1 \mathrm{H}, \mathrm{CHBrCHH}), 1.75(\mathrm{dqd}, J$ $\left.=14.0,7.5,3.9 \mathrm{~Hz}, 1 \mathrm{H}, \mathrm{CHHCH}_{3}\right), 1.49(\mathrm{dqn}, J=14.0,7.4 \mathrm{~Hz}, 1 \mathrm{H}$, $\left.\mathrm{CHHCH}_{3}\right), 1.00\left(\mathrm{t}, J=7.4 \mathrm{~Hz}, 3 \mathrm{H}, \mathrm{CH}_{2} \mathrm{CH}_{3}\right) ;{ }^{13} \mathrm{C}\left\{{ }^{1} \mathrm{H}\right\}$ NMR $(125$ $\left.\mathrm{MHz}, \mathrm{CDCl}_{3}\right) \delta 139.9\left(\mathrm{CH}_{2} \mathrm{CH}=\mathrm{CH}\right), 111.1\left(\mathrm{CH}_{2} \mathrm{CH}=\mathrm{CH}\right), 87.2$ (CHEt), $86.1 \quad\left(\mathrm{CHCH}_{2} \mathrm{CH}=\mathrm{CH}\right), \quad 82.3 \quad(\mathrm{HC} \equiv \mathrm{C}), 80.1$ $\left(\mathrm{CHClCH}_{2} \mathrm{CH}\right), 79.9(\mathrm{HC} \equiv \mathrm{C}), 78.9\left(\mathrm{CHBrCH}_{2} \mathrm{CH}\right), 59.3$ ( $\mathrm{CHCl}), 47.3(\mathrm{CHBr}), 39.3\left(\mathrm{CHBrCH}_{2}\right), 38.1\left(\mathrm{CHClCH}_{2}\right), 34.4$ $\left(\mathrm{CH}_{2} \mathrm{CH}=\mathrm{CH}\right), 25.4\left(\mathrm{CH}_{2} \mathrm{CH}_{3}\right), 10.2\left(\mathrm{CH}_{2} \mathrm{CH}_{3}\right)$; HRMS (ESITOF) $m / z[\mathrm{M}+\mathrm{Na}]^{+}$calcd for $\mathrm{C}_{15} \mathrm{H}_{20}{ }^{79} \mathrm{Br}^{35} \mathrm{ClNaO}_{2}$ 369.0227; found 369.0231, calcd for $\mathrm{C}_{15} \mathrm{H}_{20}{ }^{79 / 81} \mathrm{Br}^{37 / 35} \mathrm{ClNaO}_{2} 371.0206$; found 371.0203; $[\alpha]_{\mathrm{D}}^{25}+19.2\left(c=0.125\right.$ in $\left.\mathrm{CHCl}_{3}\right)\left\{\right.$ lit. $^{21 \mathrm{a}}[\alpha]_{\mathrm{D}}=+40.3(c$ $\left.\left.1.03 \mathrm{CHCl}_{3}\right)\right\}$.

Notoryne: Seoul Route. General Procedures. Proton $\left({ }^{1} \mathrm{H}\right)$ and carbon $\left({ }^{13} \mathrm{C}\right)$ NMR spectra were obtained on a JEOL JNM-LA300 (300/75 MHz), Bruker AV 400 (400/100 MHz), Bruker AMX 500 $(500 / 125 \mathrm{MHz})$, Bruker Avance $600(600 / 150 \mathrm{MHz})$, or Bruker Avance $900(900 / 225 \mathrm{MHz})$ spectrometer. Chemical shifts are reported in parts per million units with $\mathrm{Me}_{4} \mathrm{Si}$ or $\mathrm{CHCl}_{3}$ as the internal standard. All reactions were routinely carried out under an inert atmosphere of dry nitrogen or argon. Reactions were checked by thin layer chromatography (Kieselgel 60 F254, Merck). Spots were detected by viewing under a UV light and by colorizing with charring after immersion in a $p$-anisaldehyde solution or phosphomolybdic acid solution. In an aqueous workup, all organic solutions were dried over anhydrous sodium sulfate and filtered prior to rotary evaporation at water pump pressure. The crude compounds were purified by column chromatography on a silica gel (Kieselgel 60, 70-230 mesh, Merck). Unless otherwise noted, materials were obtained from commercial suppliers and were used without purification. All solvents were purified and dried by standard techniques just before use. THF and $\mathrm{Et}_{2} \mathrm{O}$ were freshly distilled from sodium and benzophenone. Methylene chloride, toluene, and benzene were purified by refluxing with $\mathrm{CaH}_{2}$. Hexanes and ethyl acetate were purified by simple distillation

$\left(2 R, 2^{\prime} S, 4 S, 4^{\prime} S, 5 R, 5^{\prime} R\right)$-5-(3-(Benzyloxy)propyl)-4' -bromo-4chloro-5'-ethyloctahydro-2, $2^{\prime}$-bifuran (46). To a stirred mixture of bromo alcohol $39(21.9 \mathrm{mg}, 0.055 \mathrm{mmol})$, activated silica gel, and $\mathrm{PhSeCl}(21.13 \mathrm{~mL}, 0.005 \mathrm{M})$ was added anhydrous hexane $(4 \mathrm{~mL})$. After being stirred under an argon atmosphere at room temperature for $72 \mathrm{~h}$, the mixture was filtered and concentrated in vacuo. To the resulting mixture was added $\mathrm{CH}_{3} \mathrm{CN} / \mathrm{H}_{2} \mathrm{O}(9: 1)$ solution $(3 \mathrm{~mL})$ to convert inseparable chloro ether 49 to hydroxy ether 44 . The resulting yellow solution was allowed to stand at room temperature for $24 \mathrm{~h}$. The reaction mixture was extracted with ethyl acetate $(2 \times$ $10 \mathrm{~mL}$ ). The combined organic layers were washed with saturated brine, dried over anhydrous $\mathrm{Na}_{2} \mathrm{SO}_{4}$, and concentrated in vacuo. The residue was purified by column chromatography (silica gel, $n$-hexane/ ethyl acetate, 15/1) to afford tetrahydrofuran $46(18.5 \mathrm{mg}, 80 \%)$ as a colorless oil: $R_{f} 0.67$ ( $n$-hexane/ethyl acetate, $\left.4 / 1\right) ;[\alpha]_{\mathrm{D}}^{25}=+37.5(c$ $\left.0.805, \mathrm{CHCl}_{3}\right) ;{ }^{1} \mathrm{H}$ NMR $\left(500 \mathrm{MHz}, \mathrm{CDCl}_{3}\right) \delta 7.37-7.32(\mathrm{~m}, 5 \mathrm{H})$, $4.50(\mathrm{~s}, 2 \mathrm{H}), 4.21$ (ddd, $J=6.5,6.5,7.1 \mathrm{~Hz}, 1 \mathrm{H}), 4.00-3.78(\mathrm{~m}$, $5 \mathrm{H}), 3.54-3.46(\mathrm{~m}, 2 \mathrm{H}), 2.64$ (ddd, $J=7.0,7.0,13.3 \mathrm{~Hz}, 1 \mathrm{H}), 2.28-$ $2.24(\mathrm{~m}, 2 \mathrm{H}), 2.20-2.13(\mathrm{~m}, 1 \mathrm{H}), 1.83-1.67(\mathrm{~m}, 4 \mathrm{H}), 1.65-1.45$ $(\mathrm{m}, 2 \mathrm{H}), 1.00(\mathrm{t}, J=7.5 \mathrm{~Hz}, 3 \mathrm{H}) ;{ }^{13} \mathrm{C}\left\{{ }^{1} \mathrm{H}\right\}$ NMR $\left(125 \mathrm{MHz}, \mathrm{CDCl}_{3}\right)$ $\delta 138.5,128.4,127.6,127.5,87.12,87.0,79.9,79.1,72.9,69.9,59.9$, $47.4,39.3,38.4,30.5,26.025 .410 .0$; IR (neat) 2925, 2872, 1453, $1295,1072,923 \mathrm{~cm}^{-1}$; HRMS (FAB-TOF) $\mathrm{m} / z[\mathrm{M}+\mathrm{H}]^{+}$calcd for $\mathrm{C}_{20} \mathrm{H}_{29} \mathrm{O}_{3}{ }^{79} \mathrm{Br}^{35} \mathrm{Cl}$ 431.0983; found 431.0982 .

3-((2R, 2'S, 4S, 4'S,5R, 5'R)-4'-Bromo-4-chloro-5' -ethyloctahydro[2,2'-bifuran]-5-yl)propan-1-ol. To a stirred solution of bistetrahydrofuran $46(21.9 \mathrm{mg}, 0.051 \mathrm{mmol})$ in THF $(21.13 \mathrm{~mL}, 0.005 \mathrm{M})$ was added $\mathrm{Pd}(\mathrm{OH})_{2}(4.38 \mathrm{mg}, 20 \mathrm{wt} \%$ of $\mathrm{Pd})$. The mixture was stirred under hydrogen atmosphere at room temperature for $1 \mathrm{~h}$. The mixture was filtered through a pad of Celite, and the filtrate was concentrated in vacuo. The residue was purified by column chromatography ( $n$-hexane/ethyl acetate, $2 / 1)$ to afford the title alcohol $(8.7 \mathrm{mg}, 95 \%)$ as a colorless oil: $R_{f} 0.33$ ( $n$-hexane/ethyl acetate, 2/1); $[\alpha]_{\mathrm{D}}^{25}=+48.4\left(\right.$ c $\left.1.01, \mathrm{CHCl}_{3}\right) ;{ }^{1} \mathrm{H}$ NMR $(500 \mathrm{MHz}$, $\left.\mathrm{CDCl}_{3}\right) \delta 4.26(\mathrm{ddd}, J=7.0,7.0,5.6 \mathrm{~Hz}, 1 \mathrm{H}), 4.01-3.95(\mathrm{~m}, 3 \mathrm{H})$ 3.91 (ddd, $J=7.5,7.5,4.1 \mathrm{~Hz}, 1 \mathrm{H}), 3.90-3.84(\mathrm{~m}, 1 \mathrm{H}), 3.73-3.63$ $(\mathrm{m}, 2 \mathrm{H}), 2.66(\mathrm{ddd}, J=6.6,6.6,13.6 \mathrm{~Hz}, 1 \mathrm{H}), 2.31(\mathrm{ddd}, J=7.2,7.2$, $13.6 \mathrm{~Hz}, 1 \mathrm{H}), 2.23$ (dddd, $J=13.6,6.7,4.5 \mathrm{~Hz}, 1 \mathrm{H}), 2.12$ (ddd, $J=$ 8.4, 8.4, $13.6 \mathrm{~Hz}, 1 \mathrm{H}), 1.83-1.66(\mathrm{~m}, 4 \mathrm{H}), 1.60-1.46(\mathrm{~m}, 2 \mathrm{H}), 1.00$ $(\mathrm{t}, J=7.4 \mathrm{~Hz}, 3 \mathrm{H}) ;{ }^{13} \mathrm{C}\left\{{ }^{1} \mathrm{H}\right\} \mathrm{NMR}\left(125 \mathrm{MHz}, \mathrm{CDCl}_{3}\right) \delta 87.3,87.2$, 80,0. 78.7, 62.5, 60.0, 47.2, 39.3, 37.7, 30.3, 29.4, 25.4, 9.97 ; IR (neat) $3408,2936,1648,1295,1060,971,923 \mathrm{~cm}^{-1}$; HRMS (FAB-TOF) $m / z[\mathrm{M}+\mathrm{H}]^{+}$calcd for $\mathrm{C}_{13} \mathrm{H}_{23}{ }^{79} \mathrm{Br}^{35} \mathrm{ClO}_{3}$ 341.0514; found 341.0514.

$\left(2 R, 2^{\prime} S, 4 S, 4^{\prime} S, 5 R, 5^{\prime} R\right)$-5-Allyl-4' -bromo-4-chloro-5'-ethyloctahydro-2,2'-bifuran (47-Seoul). To a solution of alcohol 3$\left(\left(2 R, 2^{\prime} S, 4 S, 4^{\prime} S, 5 R, 5^{\prime} R\right)-4^{\prime}\right.$-bromo-4-chloro-5'-ethyloctahydro- $\left[2,2^{\prime}\right.$ bifuran]-5-yl)propan-1-ol $(20.2 \mathrm{mg}, 0.059 \mathrm{mmol})$ in dry THF (5.9 $\mathrm{mL}, 0.05 \mathrm{M})$ were added $o$-nitrophenylselenocyanide $(66.98 \mathrm{mg}$, $0.045 \mathrm{mmol})$ and tri- $n$-octylphosphine $(0.263 \mathrm{~mL}$, tech. $90 \%, 0.045$ mmol) at room temperature under $\mathrm{N}_{2}$. The resulting mixture was stirred at room temperature for $10 \mathrm{~min}$. The reaction mixture was cooled to $0{ }^{\circ} \mathrm{C}$, and $30 \% \mathrm{H}_{2} \mathrm{O}_{2}(0.1 \mathrm{~mL})$ was added. The resulting mixture was stirred at the same temperature for $30 \mathrm{~min}$, allowed to warm to room temperature, and stirred for an additional $24 \mathrm{~h}$. The reaction mixture was neutralized with saturated aqueous $\mathrm{NaHCO}_{3}(1$ $\mathrm{mL})$ and diluted with diethyl ether $(4 \mathrm{~mL})$. The layers were separated, and the aqueous layer was extracted with $\mathrm{Et}_{2} \mathrm{O}(2 \times 4 \mathrm{~mL})$. The combined organic layers were washed with saturated brine, dried over anhydrous $\mathrm{Na}_{2} \mathrm{SO}_{4}$, and concentrated in vacuo. The residue was purified by column chromatography (silica gel, $n$-hexane/ethyl acetate, $25 / 1)$ to afford terminal olefin 47 -Seoul $(16.2 \mathrm{mg}, 85 \%)$ as a colorless oil: $R_{f} 0.61$ ( $n$-hexane/ethyl acetate, $\left.1 / 1\right) ;[\alpha]_{\mathrm{D}}^{25}=+66.8(c$ $\left.0.395, \mathrm{CHCl}_{3}\right) ;{ }^{1} \mathrm{H} \mathrm{NMR}\left(500 \mathrm{MHz}, \mathrm{CDCl}_{3}\right) \delta 5.83$ (dddd, $J=7.0$, 7.0, 10.3, $17.2 \mathrm{~Hz}, 1 \mathrm{H}), 5.15-5.10(\mathrm{~m}, 2 \mathrm{H}), 4.26-4.22(\mathrm{~m}, 1 \mathrm{H})$, $4.05-3.98(\mathrm{~m}, 2 \mathrm{H}), 3.96$ (ddd, $J=6.5,6.5,7.4 \mathrm{~Hz}, 1 \mathrm{H}), 3.91$ (ddd, $J$ $=4.0,7.5,7.5 \mathrm{~Hz}, 1 \mathrm{H}), 3.89-3.84(\mathrm{~m}, 1 \mathrm{H}), 2.64$ (ddd, $J=7.0,7.0$, $13.7 \mathrm{~Hz}, 1 \mathrm{H}), 2.39-2.30(\mathrm{~m}, 2 \mathrm{H}), 2.25-2.23(\mathrm{~m}, 2 \mathrm{H}), 2.17$ (ddd, $J=$ 8.6, 8.6, $13.4 \mathrm{~Hz}, 1 \mathrm{H}$ ), 1.75 (ddddd, $J=4.0,7.5,7.5,7.5,14.7 \mathrm{~Hz}$, $1 \mathrm{H}), 1.49$ (ddddd, $J=7.4,7.4,7.4,7.4,14.6 \mathrm{~Hz}, 1 \mathrm{H}), 1.00(\mathrm{t}, J=7.5$ $\mathrm{Hz}, 3 \mathrm{H}) ;{ }^{13} \mathrm{C}\left\{{ }^{1} \mathrm{H}\right\}$ NMR $\left(125 \mathrm{MHz}, \mathrm{CDCl}_{3}\right) \delta 133.3,118.0,87.1$, 86.4, 79.9, 78.9, 59.0, 47.3, 39.3, 38.1, 37.8, 25.4, 10.0; IR (neat) 2966, 2923, 1642, 1294, 1069, $920 \mathrm{~cm}^{-1}$; HRMS (FAB-TOF) $\mathrm{m} / z$ $[\mathrm{M}+\mathrm{H}]^{+}$calcd for $\mathrm{C}_{13} \mathrm{H}_{21} \mathrm{O}_{2} \mathrm{ClBrNa}$ 323.0408; found 323.0406.

((Z)-5-((2R,2'S, 4S, 4'S,5R,5'R)-4'-Bromo-4-chloro-5' -ethyloctahydro-[2,2'-bifuran]-5-yl)pent-3-en-1-yn-1-yl)triisopropylsilane (52). To a solution of terminal olefin $47-S e o u l(13.5 \mathrm{mg}, 0.038 \mathrm{mmol})$ in dry benzene $(3.64 \mathrm{~mL})$ were added enyne $50(52 \mathrm{mg}, 0.083 \mathrm{mmol})$ in 
benzene $(1.0 \mathrm{~mL})$ and Grubbs' catalyst $51(15.0 \mathrm{mg}, 0.053 \mathrm{mmol})$ in benzene $(0.2 \mathrm{~mL})$ at room temperature under nitrogen atmosphere. The reaction mixture was stirred at $70{ }^{\circ} \mathrm{C}$ for $1.5 \mathrm{~h}$. Addition of enyne $(17.3 \mathrm{mg}, 0.062 \mathrm{mmol})$ in benzene $(0.1 \mathrm{~mL})$ and Grubbs' catalyst $(4.2 \mathrm{mg}, 0.0067 \mathrm{mmol})$ in benzene $(0.1 \mathrm{~mL})$ was repeated three times every $1.5 \mathrm{~h}$. The reaction mixture was concentrated in vacuo. The residue was purified by column chromatography (silica gel, toluene) to afford enyne as a 3:1 mixture (by ${ }^{1} \mathrm{H}$ NMR analysis) of TIPS-( $Z$ )and TIPS- $(E)$-enynes $(17.2 \mathrm{mg}, 82 \%)$ as a colorless oil: $R_{f} 0.40(n-$ hexane/ethyl acetate, 10/1) (for TIPS-(Z)-enyne 52) $[\alpha]_{\mathrm{D}}^{25}=+33.4(c$ $\left.0.25, \mathrm{CHCl}_{3}\right) ;{ }^{1} \mathrm{H} \mathrm{NMR}\left(500 \mathrm{MHz}, \mathrm{CDCl}_{3}\right) \delta 6.01$ (ddd, $J=7.5,7.5$, $10.9 \mathrm{~Hz}, 1 \mathrm{H}), 5.67(\mathrm{~d}, J=10.9 \mathrm{~Hz}, 1 \mathrm{H}), 4.26-4.22(\mathrm{~m}, 1 \mathrm{H}), 4.13-$ $4.08(\mathrm{~m}, 2 \mathrm{H}), 3.97-3.94(\mathrm{~m}, 1 \mathrm{H}), 3.91$ (ddd, $J=4.0,7.5,7.5,1 \mathrm{H})$, $3.86-3.82(\mathrm{~m}, 1 \mathrm{H}), 2.72-2.57(\mathrm{~m}, 3 \mathrm{H}), 2.24-2.22(\mathrm{~m}, 2 \mathrm{H}), 2.18$ (ddd, 8.5, 8.5, $13.2 \mathrm{~Hz}, 1 \mathrm{H}$ ), 1.75 (ddddd, $J=4.0,7.4,7.4,7.4,14.8$ $\mathrm{Hz}, 1 \mathrm{H}$ ), 1.50 (ddddd, $J=7.4,7.4,7.4,7.4,14.8 \mathrm{~Hz}, 1 \mathrm{H}$ ), 1.09-1.01 $(\mathrm{m}, 3 \mathrm{H}), 1.05(\mathrm{~s}, 18 \mathrm{H}) 1.00(\mathrm{t}, J=7.4 \mathrm{~Hz}, 1 \mathrm{H}) ;{ }^{13} \mathrm{C}\left\{{ }^{1} \mathrm{H}\right\} \operatorname{NMR}(125$ $\left.\mathrm{MHz}, \mathrm{CDCl}_{3}\right) \delta 138.6,112.5,103.1,96.2,87.1,86.5,80.2,79.0,59.3$, 47.3, 39.3, 38.4, 34.7, 25.4, 18.7, 11.3, 10.0; IR (neat) 2942, 2146, 1260, 1071, $920 \mathrm{~cm}^{-1}$; HRMS (FAB-TOF) $\mathrm{m} / z[\mathrm{M}+\mathrm{H}]^{+}$calcd for $\mathrm{C}_{24} \mathrm{H}_{41}{ }^{79} \mathrm{Br}^{35} \mathrm{ClO}_{2} \mathrm{Si}$ 503.1742; found 503.1768.

(2S,2' $\left.R, 4 S, 4^{\prime} S, 5 R, 5^{\prime} R\right)$-4-Bromo-4' -chloro-5-ethyl-5'-((Z)-pent-2en-4-yn-1-yl)octahydro-2,2'-bifuran, (Z)-Notoryne ((Z)-3a). To a cooled $\left(0{ }^{\circ} \mathrm{C}\right)$ solution of $(Z)$-TIPS-enyne $52(5.2 \mathrm{mg}, 0.0103 \mathrm{mmol})$ in THF $(0.2 \mathrm{~mL})$ was added dropwise TBAF $(0.02 \mathrm{~mL}, 1.0 \mathrm{M}$ solution in THF, $0.0206 \mathrm{mmol}$ ). After the mixture was stirred at the same temperature for $1 \mathrm{~h}$, the reaction was quenched with saturated aqueous $\mathrm{NH}_{4} \mathrm{Cl}$. The resulting mixture was diluted with diethyl ether. The layers were separated, and the aqueous layer was extracted with diethyl ether $(2 \times 7 \mathrm{~mL})$. The combined organic layers were washed with brine, dried over anhydrous $\mathrm{Na}_{2} \mathrm{SO}_{4}$, and concentrated in vacuo. The residue was purified by column chromatography (silica gel, $n$ hexane/ethyl acetate, 4/1) to afford $(Z)$-notoryne $(Z)-3 a(3.4 \mathrm{mg}$, $94 \%)$ as a colorless oil: $R_{f} 0.68$ (n-hexane/ethyl acetate, $\left.1 / 1\right) ;[\alpha]_{\mathrm{D}}^{25}=$ +40.6 (c $\left.0.16 \mathrm{CHCl}_{3}\right)\left\{\right.$ lit. $^{21 \mathrm{a}}[\alpha]_{\mathrm{D}}=+40.3\left(\right.$ c $\left.\left.1.03 \mathrm{CHCl}_{3}\right)\right\} ;{ }^{1} \mathrm{H}$ NMR $\left(400 \mathrm{MHz}, \mathrm{CDCl}_{3}\right) \delta 6.08$ (dddd, $J=0.8,7.5,7.5,10.9 \mathrm{~Hz}$, $1 \mathrm{H}$ ), 5.60 (dddd, $J=1.4,1.4,2.6,10.9 \mathrm{~Hz}, 1 \mathrm{H}$ ), 4.25 (ddd, $J=5.6$, 7.1, 7.1 Hz, $1 \mathrm{H}), 4.12-4.06(\mathrm{~m}, 2 \mathrm{H}), 3.97(\mathrm{ddd}, J=5.6,6.8,8.2 \mathrm{~Hz}$, $1 \mathrm{H}), 3.91(\mathrm{ddd}, J=4.0,7.6,7.6 \mathrm{~Hz}, 1 \mathrm{H}), 3.86(\mathrm{ddd}, J=7.3,7.3,8.7$ $\mathrm{Hz}, 1 \mathrm{H}), 3.12(\mathrm{~d}, J=1.8 \mathrm{~Hz}, 1 \mathrm{H}), 2.70-2.55(\mathrm{~m}, 3 \mathrm{H}), 2.32-2.23(\mathrm{~m}$, $2 \mathrm{H}$ ), 2.17 (ddd, $J=8.4,8.4,13.2 \mathrm{~Hz}, 1 \mathrm{H}$ ), 1.75 (ddddd, $J=3.7,7.5$, 7.5, 7.5, $14.3 \mathrm{~Hz}, 1 \mathrm{H}), 1.56-1.44(\mathrm{~m}, 1 \mathrm{H}), 1.00(\mathrm{t}, J=7.4 \mathrm{~Hz}, 1 \mathrm{H})$; ${ }^{13} \mathrm{C}\left\{{ }^{1} \mathrm{H}\right\}$ NMR $\left(100 \mathrm{MHz}, \mathrm{CDCl}_{3}\right) \delta 139.9,111.1,87.2,86.2,82.3$, $80.1,80.0,79.0,59.3,47.3,39.4,38.2,34.5,25.5,10.0$; IR (neat) 3293, 2964, 1733, 1712, 1290, 1073, 967, $923 \mathrm{~cm}^{-1}$; HRMS (EI) $\mathrm{m} / z$ $\left[\mathrm{M}-\mathrm{C}_{2} \mathrm{H}_{5}\right]^{+}$calcd for $\mathrm{C}_{10} \mathrm{H}_{15}{ }^{79} \mathrm{Br}^{35} \mathrm{ClO}_{2} 280.9938$; found 280.9935 .

$\left(2 S, 2^{\prime} R, 4 S, 4^{\prime} S, 5 R, 5^{\prime} R\right)$-4-Bromo-4' -chloro-5-ethyl-5' -((E)-pent-2en-4-yn-1-yl)octahydro-2,2'-bifuran, (E)-Notoryne ((E)-3a). To a solution of terminal olefin 47 -Seoul $(7.7 \mathrm{mg}, 0.024 \mathrm{mmol})$ in dry $\mathrm{CH}_{2} \mathrm{Cl}_{2}(0.47 \mathrm{~mL}, 0.05 \mathrm{M})$ were added crotonaldehyde $(0.015 \mathrm{~mL}$, $0.19 \mathrm{mmol})$ and Grubbs' catalyst $29(2.04 \mathrm{mg}, 0.0024 \mathrm{mmol})$ at room temperature. After the mixture was stirred at $40{ }^{\circ} \mathrm{C}$ for $1.5 \mathrm{~h}$, the reaction was quenched with DMSO $(0.02 \mathrm{~mL})$. The resulting mixture was stirred at room temperature for $12 \mathrm{~h}$ and concentrated in vacuo. The residue was purified by column chromatography (silica gel, $n$ hexane/ethyl acetate, $6 / 1)$ to afford $(E)-4-\left(\left(2 R, 2^{\prime} S, 4 S, 4^{\prime} S, 5 R, 5^{\prime} R\right)-4^{\prime}-\right.$ bromo-4-chloro-5' -ethyloctahydro-[2,2'-bifuran]-5-yl)but-2-enal (7.3 $\mathrm{mg}, 87 \%)$ as a colorless oil: $R_{f} 0.37$ ( $n$-hexane/ethyl acetate, $\left.4 / 1\right)$. To a cooled $\left(-78{ }^{\circ} \mathrm{C}\right)$ solution of LDA $(0.42 \mathrm{~mL}, 0.5 \mathrm{M}$ solution in THF, $0.21 \mathrm{mmol})$ was added dropwise $\mathrm{TMSCH}_{2} \mathrm{~N}_{2}(0.105 \mathrm{~mL}, 2.0$ $\mathrm{M}$ solution in $\left.\mathrm{Et}_{2} \mathrm{O}, 0.021 \mathrm{mmol}\right)$ under argon atmosphere. After the mixture was stirred at $-78{ }^{\circ} \mathrm{C}$ for $30 \mathrm{~min}$, (E)-4$\left(\left(2 R, 2^{\prime} S, 4 S, 4^{\prime} S, 5 R, 5^{\prime} R\right)-4^{\prime}\right.$-bromo-4-chloro-5' -ethyloctahydro-[2,2'bifuran]-5-yl)but-2-enal $(7.3 \mathrm{mg}, 0.021 \mathrm{mmol})$ in THF $(0.42 \mathrm{~mL}$, $0.05 \mathrm{M})$ was added dropwise at $-78{ }^{\circ} \mathrm{C}$. After the reaction mixture was stirred at $-78{ }^{\circ} \mathrm{C}$ for $1 \mathrm{~h}$, and then at $0{ }^{\circ} \mathrm{C}$ for $1 \mathrm{~h}$, the reaction was quenched with saturated aqueous $\mathrm{NH}_{4} \mathrm{Cl}$. The layers were separated, and the aqueous layer was extracted with diethyl ether $(2 \times$ $2 \mathrm{~mL}$ ). The organic layers were washed with brine, dried over anhydrous $\mathrm{Na}_{2} \mathrm{SO}_{4}$, and concentrated in vacuo. The residue was purified by column chromatography (silica gel, $n$-hexane/ethyl acetate, $4 / 1)$ to afford $(E)$-notoryne $(E)-3 \mathrm{a}(6.4 \mathrm{mg}, 88 \%)$ as a colorless oil: $R_{f} 0.69$ (n-hexane/ethyl acetate, $\left.4 / 1\right)$; $[\alpha]_{\mathrm{D}}^{25}=+47.6(c$ $\left.0.225, \mathrm{CHCl}_{3}\right) ;{ }^{1} \mathrm{H}$ NMR $\left(400 \mathrm{MHz}, \mathrm{CDCl}_{3}\right) \delta 6.24(\mathrm{ddd}, J=7.2$, $7.2,16.0 \mathrm{~Hz}, 1 \mathrm{H}), 5.57(\mathrm{dd}, J=1.8,16.0 \mathrm{~Hz}, 1 \mathrm{H}), 4.22(\mathrm{ddd}, J=5.6$, 7.0. $7.0 \mathrm{~Hz}, 1 \mathrm{H}), 4.03-3.94(\mathrm{~m}, 3 \mathrm{H}), 3.93-3.84(\mathrm{~m}, 2 \mathrm{H}), 2.83(\mathrm{~d}, J$ $=2 \mathrm{~Hz}, 1 \mathrm{H}), 2.66(\mathrm{ddd}, J=13.2,7.0,7.0 \mathrm{~Hz}, 1 \mathrm{H}), 2.46$ (dddd, $J=$ $12.9,6.5,5.4,1.6 \mathrm{~Hz}, 1 \mathrm{H}), 2.40-2.33(\mathrm{~m}, 1 \mathrm{H}), 2.32-2.20(\mathrm{~m}, 2 \mathrm{H})$, $2.14(\mathrm{dt}, J=13.3,8.4 \mathrm{~Hz}, 1 \mathrm{H}), 1.75$ (ddddd, $J=14.1,7.5,7.5,7.5,3.8$ $\mathrm{Hz}, 1 \mathrm{H}), 1.55-1.44(\mathrm{~m}, 1 \mathrm{H}), 1.00(\mathrm{t}, J=7.4 \mathrm{~Hz}, 3 \mathrm{H}) ;{ }^{13} \mathrm{C}\left\{{ }^{1} \mathrm{H}\right\} \mathrm{NMR}$ $\left(100 \mathrm{MHz}, \mathrm{CDCl}_{3}\right) \delta 140.7,111.8,87.3,85.7,81.9,80.0,78.8$, one peak buried under $\mathrm{CDCl}_{3}$ peak by $\mathrm{HSQC}, 58.7,47.3,39.4,37.9,36.6$, 25.5, 10.0; IR (neat) 3294, 2963, 1731, 1712, 1295, 1072, 959, 925 $\mathrm{cm}^{-1}$; HRMS (EI) $\mathrm{m} / z\left[\mathrm{M}-\mathrm{C}_{2} \mathrm{H}_{5}\right]^{+}$calcd for $\mathrm{C}_{10} \mathrm{H}_{15}{ }^{79} \mathrm{Br}^{35} \mathrm{ClO}_{2}$ 280.9938; found 280.9944 .

\section{ASSOCIATED CONTENT}

\section{Supporting Information}

The Supporting Information is available free of charge on the ACS Publications website at DOI: 10.1021/acs.joc.8b02975.

Tables of comparative NMR data (PDF)

Computational methods, benchmarking, and calculated NMR parameters for all structures (PDF)

Copies of NMR spectra (PDF)

Notoryne xyz files (ZIP)

Chloroenyne xyz files (ZIP)

\section{AUTHOR INFORMATION}

\section{Corresponding Authors}

*E-mail: deukjoon@snu.ac.kr.

*E-mail: jonathan.burton@chem.ox.ac.uk.

*E-mail: robert.paton@colostate.edu.

ORCID

Deukjoon Kim: 0000-0003-4079-8734

Robert S. Paton: 0000-0002-0104-4166

Notes

The authors declare no competing financial interest.

\section{ACKNOWLEDGMENTS}

Financial support was provided by the EPSRC (UK). Q.N.N.N. acknowledges funding from the European Union's Horizon 2020 research and innovation programme under the Marie Skłodowska-Curie Grant Agreement No. 752491. We used the Dirac cluster at Oxford (EP/L015722/1); the RMACC Summit supercomputer, which is supported by the National Science foundation (ACI-1532235 and ACI1532236), the University of Colorado Boulder and Colorado State University; the Extreme Science and Engineering Discovery Environment (XSEDE) through allocation TGCHE180056. We are grateful to Prof. Gabrielle König for providing NMR spectra of the chloroenyne from L. majuscula (2b).

\section{DEDICATION}

This paper is dedicated to the memory of Erin D. Shepherd-a brilliant chemist and an amazing individual.

\section{REFERENCES}

(1) (a) Nicolaou, K. C.; Snyder, S. A. Chasing molecules that were never there: Misassigned natural products and the role of chemical synthesis in modern structure elucidation. Angew. Chem., Int. Ed. 2005, 44, 1012-1044. (b) Nicolaou, K. C.; Snyder, S. A. Chasing molecules that were never there: Misassigned natural products and 
the role of chemical synthesis in modern structure elucidation (vol 44, pg 1012, 2005). Angew. Chem., Int. Ed. 2005, 44, 2050.

(2) (a) Liu, Y.; Saurí, J.; Mevers, E.; Peczuh, M. W.; Hiemstra, H.; Clardy, J.; Martin, G. E.; Williamson, R. T. Unequivocal determination of complex molecular structures using anisotropic NMR measurements. Science 2017, 356, No. eaam5349. (b) Fujita's crystalline sponge method that allows the $\mathrm{X}$-ray structure of noncrystalline small molecules to be obtained has been applied to structure determination of noncrystalline natural products: Lee, S.; Hoshino, M.; Fujita, M.; Urban, S. Cycloelatanene A and B: absolute configuration determination and structural revision by the crystalline sponge method. Chem. Sci. 2017, 8, 1547-1550. (c) Very recently, the electron cryo-microscopy (cryoEM) method of microcrystal diffraction (MicroED) that allows the structure determination of small organic molecules, including natural products, from simple powders has been reported: Jones, C. G.; Martynowycz, M. W.; Hattne, J.; Fulton, T. J.; Stoltz, B. M.; Rodriguez, J. A.; Nelson, H. M.; Gonen, T. The CryoEM Method MicroED as a Powerful Tool for Small Molecule Structure Determination. ACS Cent. Sci. 2018, 4, 1587-1592.

(3) Bifulco, G.; Dambruoso, P.; Gomez-Paloma, L.; Riccio, R. Determination of relative configuration in organic compounds by NMR spectroscopy and computational methods. Chem. Rev. 2007, 107, 3744-3779.

(4) (a) Rychnovsky, S. D. Predicting NMR spectra by computational methods: Structure revision of hexacyclinol. Org. Lett. 2006, 8, 28952898. (b) Lodewyk, M. W.; Soldi, C.; Jones, P. B.; Olmstead, M. M.; Rita, J.; Shaw, J. T.; Tantillo, D. J. The correct structure of aquatolideexperimental validation of a theoretically-predicted structural revision. J. Am. Chem. Soc. 2012, 134, 18550-18553. (c) Tantillo, D. J. Walking in the woods with quantum chemistry-applications of quantum chemical calculations in natural products research. Nat. Prod. Rep. 2013, 30, 1079-1086. (d) Navarro-Vazquez, A. State of the art and perspectives in the application of quantum chemical prediction of ${ }^{1} \mathrm{H}$ and ${ }^{13} \mathrm{C}$ chemical shifts and scalar couplings for structural elucidation of organic compounds. Magn. Reson. Chem. 2017, 55, 29-32.

(5) (a) Michels, T. D.; Dowling, M. S.; Vanderwal, C. D. A synthesis of echinopine B. Angew. Chem., Int. Ed. 2012, 51, 7572-7576. (b) Shi, H.; Michaelides, I. N.; Darses, B.; Jakubec, P.; Nguyen, Q. N. N.; Paton, R. S.; Dixon, D. J. Total Synthesis of (-)-Himalensine A. J. Am. Chem. Soc. 2017, 139, 17755-17758.

(6) Nguyen, Q. N. N.; Schwochert, J.; Tantillo, D. J.; Lokey, R. S. Using (1)H and (13)C NMR chemical shifts to determine cyclic peptide conformations: a combined molecular dynamics and quantum mechanics approach. Phys. Chem. Chem. Phys. 2018, 20, 1400314012 .

(7) Wu, J.; Lorenzo, P.; Zhong, S.; Ali, M.; Butts, C. P.; Myers, E. L.; Aggarwal, V. K. Synergy of synthesis, computation and NMR reveals correct baulamycin structures. Nature 2017, 547, 436-440.

(8) Bootwicha, T.; Feilner, J. M.; Myers, E. L.; Aggarwal, V. K. Iterative assembly line synthesis of polypropionates with full stereocontrol. Nat. Chem. 2017, 9, 896-902.

(9) (a) Sohn, T. I.; Kim, D.; Paton, R. S. Substrate-Controlled Asymmetric Total Syntheses of Microcladallenes A, B, and C Based on the Proposed Structures. Chem. - Eur. J. 2015, 21, 15988-15997. (b) Jeong, D.; Sohn, T. I.; Kim, J. Y.; Kim, G.; Kim, D.; Paton, R. S. Construction of 6,10-syn- and -anti-2,5-dioxabicyclo[2.2.1]heptane skeletons via oxonium ion formation/fragmentation: prediction of structure of (E)-ocellenyne by NMR calculation. Org. Lett. 2017, 19, 6252-6255.

(10) Smith, S. G.; Goodman, J. M. Assigning the Stereochemistry of Pairs of Diastereoisomers Using GIAO NMR Shift Calculation. J. Org. Chem. 2009, 74, 4597-4607.

(11) Smith, S. G.; Goodman, J. M. Assigning Stereochemistry to Single Diastereoisomers by GIAO NMR Calculation: The DP4 Probability. J. Am. Chem. Soc. 2010, 132, 12946-12959.

(12) (a) Ermanis, K.; Parkes, K. E.; Agback, T.; Goodman, J. M. Expanding DP4: application to drug compounds and automation. Org.
Biomol. Chem. 2016, 14, 3943-3949. (b) Ermanis, K.; Parkes, K. E. B.; Agback, T.; Goodman, J. M. Doubling the power of DP4 for computational structure elucidation. Org. Biomol. Chem. 2017, 15, 8998-9007.

(13) CHESHIRE, CHEmical SHift REpository with Coupling Constants Added Too; http://cheshirenmr.info.

(14) (a) Grimblat, N.; Zanardi, M. M.; Sarotti, A. M. Beyond DP4: an Improved Probability for the Stereochemical Assignment of Isomeric Compounds using Quantum Chemical Calculations of NMR Shifts. J. Org. Chem. 2015, 80, 12526-12534. (b) Sarotti, A. M.; Pellegrinet, S. C. A multi-standard approach for GIAO (13)C NMR calculations. J. Org. Chem. 2009, 74, 7254-7260. (c) Andrews, K. G.; Spivey, A. C. Improving the accuracy of computed (13)C NMR shift predictions by specific environment error correction: fragment referencing. J. Org. Chem. 2013, 78, 11302-11317.

(15) (a) Sheldrake, H. M.; Jamieson, C.; Burton, J. W. The changing faces of halogenated marine natural products: Total synthesis of the reported structures of elatenyne and an enyne from Laurencia majuscula. Angew. Chem., Int. Ed. 2006, 45, 7199-7202. (b) Sheldrake, H. M.; Jamieson, C.; Pascu, S. I.; Burton, J. W. Synthesis of the originally proposed structures of elatenyne and an enyne from Laurencia majuscula. Org. Biomol. Chem. 2009, 7, 238-252. (c) Smith, S. G.; Paton, R. S.; Burton, J. W.; Goodman, J. M. Stereostructure assignment of flexible five-membered rings by GIAO (13)C NMR calculations: Prediction of the stereochemistry of elatenyne. J. Org. Chem. 2008, 73, 4053-4062. (d) Dyson, B. S.; Burton, J. W.; Sohn, T. I.; Kim, B.; Bae, H.; Kim, D. Total synthesis and structure confirmation of elatenyne: success of computational methods for NMR prediction with highly flexible diastereomers. J. Am. Chem. Soc. 2012, 134, 11781-11790. (e) In 2016, the absolute configuration of elatenyne was confirmed by X-ray crystallography using Fujita's crystalline sponge method: Urban, S.; Brkljaca, R.; Hoshino, M.; Lee, S.; Fujita, M. Determination of the Absolute Configuration of the Pseudo-Symmetric Natural Product Elatenyne by the Crystalline Sponge Method. Angew. Chem., Int. Ed. 2016, 55, 2678-2682.

(16) (a) Shepherd, D. J.; Broadwith, P. A.; Dyson, B. S.; Paton, R. S.; Burton, J. W. Structure Reassignment of Laurefurenynes A and B by Computation and Total Synthesis. Chem. - Eur. J. 2013, 19, 1264412648. (b) For a strategically different synthesis of laurefurenyne A, see: Holmes, M. T.; Britton, R. Total synthesis and structural revision of laurefurenynes A and B. Chem. - Eur. J. 2013, 19, 12649-12652.

(17) Wright, A. D.; Konig, G. M.; Denys, R.; Sticher, O. Seven New Metabolites From The Marine Red Alga Laurencia Majuscula. J. Nat. Prod. 1993, 56, 394-401.

(18) Gopichand, Y.; Schmitz, F. J.; Shelley, J.; Rahman, A.; Van der Helm, D. Marine natural products: halogenated acetylenic ethers from the sea hare Aplysia dactylomela. J. Org. Chem. 1981, 46, 5192-5197.

(19) Hall, J. G.; Reiss, J. A. Elatenyne - a Pyrano[3,2-b]pyranyl Vinyl Acetylene from the Red Alga Laurencia elata. Aust. J. Chem. 1986, 39, 1401-1409.

(20) For the total synthesis of (E)- and (Z)-elatenyne and of laurendecumenyne $\mathrm{B}$, see ref $15 \mathrm{~d}$.

(21) (a) Kikuchi, H.; Suzuki, T.; Kurosawa, E.; Suzuki, M. The Structure Of Notoryne, A Halogenated C15 Nonterpenoid With A Novel Carbon Skeleton From The Red Alga Laurencia Nipponica Yamada. Bull. Chem. Soc. Jpn. 1991, 64, 1763-1775. (b) 3(E)Notoryne has also been reported as a natural product: Fukuzawa, A.; Aye, M.; Nakamura, M.; Tanura, M.; Murai, A. Structure elucidation of laureoxanyne, a new nonisoprenoid C-15-enyne, using lactoperoxidase. Tetrahedron Lett. 1990, 31, 4895-4898.

(22) (a) Ji, N. Y.; Li, X. M.; Li, K.; Wang, B. G. Laurendecumallenes A-B and laurendecumenynes A-B, halogenated nonterpenoid C-15Acetogenins from the marine red alga Laurencia decumbens. J. Nat. Prod. 2007, 70, 1499-1502. (b) Ji, N. Y.; Li, X. M.; Li, K.; Wang, B. G. Laurendecumallenes A-B and Laurendecumenynes A-B, Halogenated Nonterpenoid C(15)-Acetogenins from the Marine Red Alga Laurencia decumbens. (vol 70, pg 1499, 2007). J. Nat. Prod. 2010, 73, 1192. 
(23) Abdel-Mageed, W. M.; Ebel, R.; Valeriote, F. A.; Jaspars, M. Laurefurenynes A-F, new Cyclic Ether Acetogenins from a Marine Red Alga, Laurencia sp. Tetrahedron 2010, 66, 2855-2862.

(24) Murai, A. Biosynthesis of Cyclic Bromoethers from Red Algae. In Comprehensive Natural Products Chemistry; Barton, D. H. R., MethCohn, O., Nakinishi, K., Eds.; Elsevier: Oxford, 1999; Vol. 1, pp 303324.

(25) (a) Kurosawa, E.; Irie, T.; Fukuzawa, A. trans-Laurediol and cislaurediol, unsaturated glycols from Laurencia Nipponica yamada. Tetrahedron Lett. 1972, 13, 2121-2124. (b) Fukuzawa, A.; Honma, T.; Takasugi, Y.; Murai, A. Biogenetic intermediates, $(3 E$ and $3 Z, 12 Z)$-laurediols and (3E and 3Z)-12,13-dihydrolaurediols, isolated from Laurencia Nipponica. Phytochemistry 1993, 32, 1435-1438.

(26) For reviews of halogenating enzymes, see: (a) Butler, A.; Carter-Franklin, J. N. The role of vanadium bromoperoxidase in the biosynthesis of halogenated marine natural products. Nat. Prod. Rep. 2004, 21, 180-188. (b) Vaillancourt, F. H.; Yeh, E.; Vosburg, D. A.; Garneau-Tsodikova, S.; Walsh, C. T. Nature's inventory of halogenation catalysts: Oxidative strategies predominate. Chem. Rev. 2006, 106, 3364-3378.

(27) Braddock, D. C. A hypothesis concerning the biosynthesis of the obtusallene family of marine natural products via electrophilic bromination. Org. Lett. 2006, 8, 6055-6058.

(28) (a) McPhail, K. L.; Davies-Coleman, M. T. (3Z)-bromofucin from a South African sea hare. Nat. Prod. Res. 2005, 19, 449-452. (b) Coll, J. C.; Wright, A. D. Tropical Marine-Algae. IV. Novel Metabolites from the Red Alga Laurencia implicata (Rhodophyta, Rhodophyceae, Ceramiales, Rhodomelaceae). Aust. J. Chem. 1989, 42, 1685-1693. (c) For a recent biomimetic synthesis of the bromo- and chlorofucins, see: Kim, B.; Sohn, T. I.; Kim, D.; Paton, R. S. Asymmetric Total Syntheses and Structure Confirmation of Chlorofucins and Bromofucins. Chem. - Eur. J. 2018, 24, 2634-2642.

(29) The configurations of the natural products obtusenyne, laurepinnacin, and isolaurepinnacin are consistent with them being biosynthetically derived from a $12(Z)$-laurediol derivative: (a) King, T. J.; Imre, S.; Öztunc, A.; Thomson, R. H. Obtusenyne, a new acetylenic nine-membrered cyclic ether from Laurenica obtusa. Tetrahedron Lett. 1979, 20, 1453-1454. (b) Howard, B. M.; Schulte, G. R.; Fenical, W.; Solheim, B.; Clardy, J. Three new vinyl acetylenes from the marine red alga Laurencia. Tetrahedron 1980, 36, 1747-1751. (c) Fukuzawa, A.; Masamune, T. Laurepinnacin and isolaurepinnacin, new acetylenic cyclic ethers from the marine red alga Laurencia pinnata Yamada. Tetrahedron Lett. 1981, 22, 4081-4084.

(30) For alternative biosynthetic postulates, see: (a) Snyder, S. A.; Treitler, D. S.; Brucks, A. P.; Sattler, W. A general strategy for the stereocontrolled preparation of diverse 8- and 9-membered laurenciatype bromoethers. J. Am. Chem. Soc. 2011, 133, 15898-15901. (b) Bonney, K. J.; Braddock, D. C. A unifying stereochemical analysis for the formation of halogenated C15-acetogenin medium-ring ethers from Laurencia species via intramolecular bromonium ion assisted epoxide ring-opening and experimental corroboration with a model epoxide. J. Org. Chem. 2012, 77, 9574-9584.

(31) (a) Lodewyk, M. W.; Siebert, M. R.; Tantillo, D. J. Computational prediction of ${ }^{1} \mathrm{H}$ and ${ }^{13} \mathrm{C}$ chemical shifts: a useful tool for natural product, mechanistic, and synthetic organic chemistry. Chem. Rev. 2012, 112, 1839-1862. (b) Nguyen, Q. N.; Tantillo, D. J. The many roles of quantum chemical predictions in synthetic organic chemistry. Chem. - Asian J. 2014, 9, 674-680. (c) Willoughby, P. H.; Jansma, M. J.; Hoye, T. R. A guide to small-molecule structure assignment through computation of $((1) \mathrm{H}$ and (13)C) NMR chemical shifts. Nat. Protoc. 2014, 9, 643-660. (d) Jackson, K. E.; Paton, R. S. A Guide to Small-Molecule Structure Assignment through Computation of $\left({ }^{1} \mathrm{H}\right.$ and $\left.{ }^{13} \mathrm{C}\right)$ NMR Chemical Shifts. In Applied Theoretical Organic Chemistry; Tantillo, D. J., Ed.; World Scientific, 2018; Vol. 1, pp 165-189.

(32) (a) Halgren, T. A. Merck molecular force field. I. Basis, form, scope, parameterization, and performance of MMFF94. J. Comput. Chem. 1996, 17, 490-519. (b) We used a 10000 step Monte Carlo multiple minimum (MCMM) search for each diastereomer: Chang,
G.; Guida, W. C.; Still, W. C. An internal-coordinate Monte Carlo method for searching conformational space. J. Am. Chem. Soc. 1989, 111, 4379-4386 as implemented in Macromodel (Schrödinger, LLC, New York, NY, 2018.

(33) Jackson, K.; Jaffar, S. K.; Paton, R. S. Computational organic chemistry. Annu. Rep. Prog. Chem., Sect. B: Org. Chem. 2013, 109, 235-255.

(34) (a) Chai, J. D.; Head-Gordon, M. Long-range corrected hybrid density functionals with damped atom-atom dispersion corrections. Phys. Chem. Chem. Phys. 2008, 10, 6615-6620. (b) Frisch, M. J.; et al. Gaussian 09, revision D.01; Gaussian, Inc.: Wallingford, CT, 2009.

(35) Cossi, M.; Rega, N.; Scalmani, G.; Barone, V. Energies, structures, and electronic properties of molecules in solution with the C-PCM solvation model. J. Comput. Chem. 2003, 24, 669-681.

(36) (a) Riplinger, C.; Sandhoefer, B.; Hansen, A.; Neese, F. Natural triple excitations in local coupled cluster calculations with pair natural orbitals. J. Chem. Phys. 2013, 139, 134101-13413. (b) We used Orca 4.0: Neese, F. Software Update: The ORCA Program System, Version 4.0. Wiley Interdiscip. Rev. Comput. Mol. Sci. 2018, 8, No. e1327.

(37) (a) Pyykkö, P.; Görling, A.; Rösch, N. A transparent interpretation of the relativistic contribution to the N.M.R. "heavy atom chemical shift'. Mol. Phys. 1987, 61, 195-205. (b) Kutateladze, A. G.; Reddy, D. S. High-Throughput in Silico Structure Validation and Revision of Halogenated Natural Products Is Enabled by Parametric Corrections to DFT-Computed (13)C NMR Chemical Shifts and Spin-Spin Coupling Constants. J. Org. Chem. 2017, 82, $3368-3381$.

(38) Braddock, D. C.; Rzepa, H. S. Structural reassignment of obtusallenes V, VI, and VII by GIAO-based density functional prediction. J. Nat. Prod. 2008, 71, 728-730.

(39) (a) Marell, D. J.; Emond, S. J.; Kulshrestha, A.; Hoye, T. R. Analysis of seven-membered lactones by computational NMR methods: proton NMR chemical shift data are more discriminating than carbon. J. Org. Chem. 2014, 79, 752-758. (b) Chini, M. G.; Riccio, R.; Bifulco, G. Computational NMR Methods in the Stereochemical Analysis of Organic Compounds: Are Proton or Carbon NMR Chemical Shift Data More Discriminating? Eur. J. Org. Chem. 2015, 2015, 1320-1324.

(40) Blakemore, P. R.; Cole, W. J.; Kocienski, P. J.; Morley, A. A Stereoselective Synthesis of trans-1,2-Disubstituted Alkenes Based on the Condensation of Aldehydes with Metallated 1-Phenyl-1Htetrazol-5-yl Sulfones. Synlett 1998, 1998, 26-28.

(41) Crimmins, M. T.; Powell, M. T. Enantioselective total synthesis of (+)-obtusenyne. J. Am. Chem. Soc. 2003, 125, 7592-7595.

(42) For a review of the mechanisms of the Julia-Kocienski reaction, see: Aïssa, C. Mechanistic Manifold and New Developments of the Julia-Kocienski Reaction. Eur. J. Org. Chem. 2009, 2009, 18311844.

(43) The geometry of the double bond was assigned as $(E)$ on the basis of the ${ }^{13} \mathrm{C}$ NMR chemical shifts of the allylic carbon atoms $(\delta=$ 36.1, $34.1 \mathrm{ppm}$ ), see: Crews, P.; Rodríguez, J.; Jaspars, M. Organic Structure Analysis, 2nd ed.; Oxford University Press: New York, 2010; pp 59-60, and by comparison with closely related compounds refs $15 \mathrm{~d}$ and 16a.

(44) (a) Sharpless, K. B.; Amberg, W.; Bennani, Y. L.; Crispino, G. A.; Hartung, J.; Jeong, K. S.; Kwong, H. L.; Morikawa, K.; Wang, Z. $\mathrm{M}$. The osmium-catalyzed asymmetric dihydroxylation: a new ligand class and a process improvement. J. Org. Chem. 1992, 57, 2768-2771. (b) Kolb, H. C.; Vannieuwenhze, M. S.; Sharpless, K. B. Catalytic Asymmetric Dihydroxylation. Chem. Rev. 1994, 94, 2483-2547.

(45) Nicolaou, K. C.; Nadin, A.; Leresche, J. E.; La Greca, S.; Tsuri, T.; Yue, E. W.; Yang, Z. Synthesis of the First Fully Functionalized Core of the Zaragozic Acids/Squalestatins. Angew. Chem., Int. Ed. Engl. 1994, 33, 2187-2190.

(46) Ohtani, I.; Kusumi, T.; Kashman, Y.; Kakisawa, H. High-field FT NMR application of Mosher's method. The absolute configurations of marine terpenoids. J. Am. Chem. Soc. 1991, 113, 40924096. 
(47) (a) Han, J. S.; Lowary, T. L. Total synthesis of buergerinin F and buergerinin G. J. Org. Chem. 2003, 68, 4116-4119. (b) Takao, K.; Yasui, H.; Yamamoto, S.; Sasaki, D.; Kawasaki, S.; Watanabe, G.; Tadano, K. Asymmetric total syntheses of $(+)$-mycoepoxydiene and related natural product (-)-1893A: Application of one-pot ringopening/cross/ring-closing metathesis to construct their 9-oxabicyclo 4.2.1 nona-2,4-diene skeleton. J. Org. Chem. 2004, 69, 8789-8795. (c) Some reduced product was also formed where the iodine atom had been replaced by a hydrogen atom.

(48) (a) Freeman, P. K.; Hutchinson, L. L. Alkyllithium reagents from alkyl halides and lithium radical anions. J. Org. Chem. 1980, 45, 1924-1930. (b) Ireland, R. E.; Smith, M. G. 3-Acyltetramic Acid Antibiotics. 2. Synthesis of (+)-Streptolic Acid. J. Am. Chem. Soc. 1988, 110, 854-860.

(49) Appel, R. Tertiary Phosphane/Tetrachloromethane, a Versatile Reagent for Chlorination, Dehydration, and P-N Linkage. Angew. Chem., Int. Ed. Engl. 1975, 14, 801-811.

(50) (a) Kim, B.; Lee, M.; Kim, M. J.; Lee, H.; Kim, S.; Kim, D.; Koh, M.; Park, S. B.; Shin, K. J. Biomimetic asymmetric total synthesis of (-)-laurefucin via an organoselenium-mediated intramolecular hydroxyetherification. J. Am. Chem. Soc. 2008, 130, 16807-16811. (b) Kim, H.; Lee, H.; Lee, D.; Kim, S.; Kim, D. Asymmetric total syntheses of $(+)-3-(Z)$-laureatin and (+)-3-(Z)-isolaureatin by "lone pair-lone pair interaction-controlled" isomerization. J. Am. Chem. Soc. 2007, 129, 2269-2274.

(51) Burton, J. W.; Clark, J. S.; Derrer, S.; Stork, T. C.; Bendall, J. G.; Holmes, A. B. Synthesis of medium ring ethers. 5. The synthesis of (+)-laurencin. J. Am. Chem. Soc. 1997, 119, 7483-7498.

(52) (a) Mitsunobu, O.; Yamada, M. Preparation of Esters of Carboxylic and Phosphoric Acid via Quaternary Phosphonium Salts. Bull. Chem. Soc. Jpn. 1967, 40, 2380-2382. (b) Martin, S. F.; Dodge, J. A. Efficacious Modification of the Mitsunobu Reaction for the Inversoins of Sterically Hindered Secondary Alcohols. Tetrahedron Lett. 1991, 32, 3017-3020. (c) Proctor, A. J.; Beautement, K.; Clough, J. M.; Knight, D. W.; Li, Y. F. Chromatography-free product separation in the Mitsunobu reaction. Tetrahedron Lett. 2006, 47, $5151-5154$.

(53) Donohoe, T. J.; House, D. Ammonia Free Partial Reduction of Aromatic Compounds Using Lithium Di-tert-butylbiphenyl (LiDBB). J. Org. Chem. 2002, 67, 5015-5018.

(54) In the absence of a proton donor, the benzyl ether $\mathbf{3 3}$ was formed as the major product.

(55) (a) DFT calculations had predicted $2 \mathbf{b}$ to be the actual structure of the natural product. We therefore first synthesized $\mathbf{2 b}$ before preparing diastereomer $\mathbf{2 d}$. Hence, our synthesis of diastereomer 2d involves two Mitsunobu reactions. (b) The Mitsunobu reaction and subsequent methanolysis for the praparation of $\mathbf{2 d}$ from $\mathbf{2 b}$ were conducted on very small scale, and hence yields have not been quoted.

(56) The first synthesis of notoryne, the "Seoul" synthesis described herein, was first reported in 2009. See: Lee, M. First Asymmetric Total Synthesis of (Z)- and (E)-Notorynes. M.S. Thesis, Seoul National University, Seoul, Korea, 2009. For a very recent total synthesis of (Z)-notoryne, see: Senapati, S.; Das, S.; Ramana, C. V. Total Synthesis of Notoryne. J. Org. Chem. 2018, 83, 12863-12868.

(57) Hooz, J.; Gilani, S. S. H. A rapid, mild procedure for the preparation of alkyl chlorides and bromides. Can. J. Chem. 1968, 46, 86-87.

(58) The spectroscopic data for compound $\mathbf{4 4}$ can be found in the Supporting Information of ref 50 a.

(59) (a) Aliev, A. E.; Harris, K. D. M. $37 \mathrm{Cl} / 35 \mathrm{Cl}$ isotope effects in 13C NMR spectroscopy of chlorohydrocarbons. Magn. Reson. Chem. 1993, 31, 54-57. (b) For a recent example of the use of ${ }^{13} \mathrm{C}$ NMR chlorine-induced isotopic shift to determine the position fo a chlorine atom, see: Braddock, D. C.; Bhuva, R.; Millan, D. S.; Perez-Fuertes, Y.; Roberts, C. A.; Sheppard, R. N.; Solanki, S.; Stokes, E. S. E.; White, A. J. P. A biosynthetically-inspired synthesis of the tetrahydrofuran core of obtusallenes II and IV. Org. Lett. 2007, 9, 445-448.
(60) Yamakado, Y.; Ishiguro, M.; Ikeda, N.; Yamamoto, H. Stereoselective Carbonyl Olefination via Organosilicon Compounds. J. Am. Chem. Soc. 1981, 103, 5568-5570.

(61) Lee, H.; Kim, K. W.; Park, J.; Kim, H.; Kim, S.; Kim, D.; Hu, X. Q.; Yang, W. T.; Hong, J. Y. A general strategy for construction of both 2,6-cis- and 2,6-trans-disubstituted tetrahydropyrans: Substratecontrolled asymmetric total synthesis of (+)-scanlonenyne. Angew. Chem., Int. Ed. 2008, 47, 4200-4203.

(62) Some resonances in the ${ }^{13} \mathrm{C}$ NMR spectrum of $(Z)$-notoryne had originally been misassigned, and the total synthesis of the natural product allowed complete assignment of the ${ }^{13} \mathrm{C}$ NMR spectrum of the natural product (and see ref 56). 